\title{
Insights on the Evolution of Metabolic Networks of Unicellular Translationally Biased Organisms from Transcriptomic Data and Sequence Analysis
}

\author{
Alessandra Carbone, ${ }^{1}$ Richard Madden ${ }^{2}$ \\ ${ }^{1}$ Génomique Analytique, Université Pierre et Marie Curie, INSERM U511, 91 Bd de l'Hôpital, 75013 Paris, France \\ ${ }^{2}$ Institut des Hautes Études Scientifiques, 35 route de Chartres, 91440 Bures-sur-Yvette, France \\ Received: 9 November 2004 / Accepted: 20 April 2005 [Reviewing Editor: Dr. Richard Kliman]
}

\begin{abstract}
Codon bias is related to metabolic functions in translationally biased organisms, and two facts are argued about. First, genes with high codon bias describe in meaningful ways the metabolic characteristics of the organism; important metabolic pathways corresponding to crucial characteristics of the lifestyle of an organism, such as photosynthesis, nitrification, anaerobic versus aerobic respiration, sulfate reduction, methanogenesis, and others, happen to involve especially biased genes. Second, gene transcriptional levels of sets of experiments representing a significant variation of biological conditions strikingly confirm, in the case of Saccharomyces cerevisiae, that metabolic preferences are detectable by purely statistical analysis: the high metabolic activity of yeast during fermentation is encoded in the high bias of enzymes involved in the associated pathways, suggesting that this genome was affected by a strong evolutionary pressure that favored a predominantly fermentative metabolism of yeast in the wild. The ensemble of metabolic pathways involving enzymes with high codon bias is rather well defined and remains consistent across many species, even those that have not been considered as translationally biased, such as Helicobacter pylori, for instance, reveal some weak form of translational bias for this genome. We provide numerical evidence, supported by experimental data, of these facts and conclude that
\end{abstract}

This article contains online-only suppplementary material. This material can also be found at http://www.ihes.fr/ carbone/data.htm.

Correspondence to: Alessandra Carbone; email: carbone@ihes.fr the metabolic networks of translationally biased genomes, observable today as projections of eons of evolutionary pressure, can be analyzed numerically and predictions of the role of specific pathways during evolution can be derived. The new concepts of Comparative Pathway Index, used to compare organisms with respect to their metabolic networks, and Evolutionary Pathway Index, used to detect evolutionarily meaningful bias in the genetic code from transcriptional data, are introduced.

Key words: Codon bias - Metabolic networks Unicellular organisms - Codon Adaptation Index - Transcriptomic data

\section{Introduction}

In silico methods capable of indicating the metabolism and lifestyle of an organism and explaining its evolution in the wild would be an invaluable tool, especially for organisms for which these characteristics are still unknown. High expression rates of certain genes during exponential growth, or of enzymes involved in fundamental metabolic activities like glycolysis, have been very often reported in the literature for fast growers and studied, since the 1980s, through the notion of the Codon Adaptation Index (CAI) (Sharp and Li 1987). For fast-growing organisms like Escherichia coli and Saccharomyces cerevisiae, but also Caenorhabditis elegans and Dro- 
sophila melanogaster, genes have been ranked by CAI values and correlated with gene expression levels and functional activity (Sharp and Li 1987; Sharp et al. 1986, 1988; Shields and Sharp 1987; Stenico et al. 1994). Carbone et al. (2003) showed that the CAI can be defined purely by sequence analysis, that it can be calculated for genomes of organisms whose biological activities are not known, and that the resulting ranking of genes correlates well with the dominant codon bias of the organism. This bias need not be related to translational efficiency as for fast growers, but can have any other nature (such as compositional, as for AT- and GC-skew bias, or physical, e.g., strand bias). Exploiting the fact that CAI is a universal measure for analyzing dominant codon bias of any origin in organisms which are not necessarily translationally biased, one can numerically evaluate the strength of different codon biases for an organism and classify genes accordingly to dominant biases (Carbone et al. 2004). For translationally biased organisms, where gene ranking based on CAI has immediate biological interpretation, one can shift the paradigm of the 1980 s, which is applied to genes, to groups of enzymes and their metabolic activity, instead, and approach, in a systematic way, the classification of metabolic pathways distinguishing those which are essential for the life cycle of the organism from those which are rarely activated but employed to survive under specific environmental conditions. Namely, through the new notion of the Relative Pathway Index, we rank metabolic pathways and correlate them with the importance of their metabolic function for the life of the organism through evolution. Metabolic pathways that are constitutive in the everyday life of the organism and metabolic pathways that need to be activated rapidly under specific conditions are well characterized by the analysis. Strong evolutionary pressures which favored a given metabolism of an organism in the wild are also characterized in the analysis.

Several energy metabolism pathways turn out to be constituted by enzymes with high codon bias in translationally biased organisms known to be driven by very different physiologies: for instance, the highly biased codon composition of glycolytic enzymes is strikingly evident in fast-growing aerobic bacteria and in S. cerevisiae, and the key role of photosynthetic pathways for Synechocystis (Mrázek et al. 2001) or of methane metabolism for Methanosarcina acetivorans can be identified by the high codon bias of their enzymes. Important metabolic pathways can be detected for organisms which are not necessarily fast growing and whose genomes might have a homogeneous codon composition even though the signal is weaker. For instance, a highly biased codon composition of glycolytic enzymes is present in Mycobacterium tuberculosis H37Rv (mtbRv) whose genome displays only a weak form of translation bias (Carbone et al. 2004), and more surprisingly in $\mathrm{He}$ licobacter pylori (hpy), a genome of rather homogeneous codon composition (Lafay et al. 2000), where glycolytic enzymes are biased above average. This finding suggests that translational selection affected hpy.

In general, given an organism or groups of organisms whose genomes have been affected by some (weak or strong) form of translational selection and have a similar lifestyle, we show that genetic coding is tuned, favoring specific pathways and disfavoring others. These coding features hold across organisms and detecting them allows us to predict or confirm the lifestyle of an organism. This is done with the help of the new concept of the Comparative Pathway Index, which enables organism comparisons based on their metabolic activities. Several examples, supported by experimental evidence, have been chosen to illustrate how well the method works in view of its application to subsequent analysis of poorly studied organisms.

To validate our statistical analysis, we consider the metabolic activities of $S$. cerevisiae reported by transcriptomic data under sufficiently different biological conditions and compare them with the classification of metabolic pathways obtained by sequence analysis. Because changes in gene expression over sufficiently long periods of time and variations of biological conditions might be complex and might involve the integration of many kinds of information on the nutritional and metabolic state of the cell, we consider mRNA abundance collected during the $S$. cerevisiae cell cycle under diauxic shift (deRisi et al. 1997) (here glucose quantities decrease in the medium during the cell cycle and induce the yeast to move from fermentation to aerobic respiration), and we analyze the yeast metabolic network through these data. A classification of metabolic pathways based on transcriptomic data and on the new concept of the Evolutionary Pathway Index is proposed. The comparison between this metabolic classification and the one obtained through sequence analysis suggests that the coding sequence of enzymes involved in specific metabolic functions contains information on the physiological responses of an organism during evolutionarily favored conditions. Namely, we argue in favor of the fact that in $S$. cerevisiae the biasing of genes involved in the fermentative state corresponds to a strong evolutionary pressure favoring this fermentative metabolic state of yeast in the wild (Wagner 2000). The high transcription of enzymes involved in highly active pathways during fermentation (and possibly not during aerobic respiration) correlates particularly well with the high CAI value of these enzymes. This observation is supported also by the available transcriptomic data 
Table 1. List of organisms considered for metabolic pathway comparison analysis

\begin{tabular}{lllllllllllll}
\hline Organism & Sign & & & DT & P & ER & CP & CER & $\mu$ M & $\sigma$ M & Min & Max \\
\hline Agrobacterium tumefaciens (agro) & + & + & $\times$ & $2 \mathrm{~h}$ & 156 & 917 & 30 & 72 & 0.53 & 0.06 & 0.30 & 0.77 \\
Bacillus subtilis (bsub) & + & - & - & $60 \mathrm{~m}$ & 104 & 754 & 27 & 63 & 0.41 & 0.06 & 0.32 & 0.58 \\
Escherichia coli (ecoli) & + & - & - & $30 \mathrm{~m}$ & 148 & 2908 & 34 & 96 & 0.40 & 0.07 & 0.25 & 0.64 \\
Haemophilus influenzae (hin) & + & - & + & $30 \mathrm{~m}$ & 88 & 565 & 29 & 62 & 0.43 & 0.06 & 0.31 & 0.63 \\
Vibrio cholera (vcho) & + & - & - & $30 \mathrm{~m}$ & 170 & 852 & 32 & 90 & 0.36 & 0.07 & 0.22 & 0.63 \\
Saccharomyces cerevisiae (yeast) & + & - & - & $90 \mathrm{~m}$ & 86 & 599 & 20 & 39 & 0.31 & 0.12 & 0.15 & 0.73 \\
\hline
\end{tabular}

Note. On the right of each organism, three symbols $(+,-, \times)$ are used to represent in short the codon bias signature of the organism. Columns are interpreted as follows: (1) +, translational bias; (2) +, GC3 content; (3) +, strand bias; $\times$, no replication origin is known. (-) No bias is present. No GC-skew bias or AT-skew bias is detected for these six organisms. Doubling time (DT) given in minutes (m) or hours (h); number of metabolic pathways (P) included in BioCyc and of enzymatic reactions (ER) involved in these pathways; number of metabolic pathways constituted by enzymes whose corresponding genes are clustered along the genome sequence (CP); and number of enzymatic reactions involved in such pathways (CER). Mean $(\mu M)$, standard deviation $(\sigma M)$, minimum and maximum PI(P), for pathways $\mathrm{P}$ in the metabolic network $\mathrm{M}$.

on seripauperine proteins (Holstege et al. 1998; James et al. 2003; Viswanathan et al. 1994) and on Hem13 proteins (Amillet et al. 1995).

Our results open a way of exploring evolutionary pressure and natural selection for organisms grown in the wild, understanding the need for different levels of gene regulation in unicellular organisms, and, hopefully, predicting the metabolic activities of translationally biased organisms, as well as suggesting the best conditions for growth in the laboratory. Similar questions have been addressed by Akashi and Gojobori (2002), who report that metabolic efficiency is related to amino acid composition for the genomes of E. coli and B. subtilis.

\section{Materials and Methods}

\section{Organisms and Genomes}

An analysis of metabolic pathways has been done for the translationally biased organisms listed in Table 1 plus Pyrococcus abissi, Synechocystis, Methanobacterium thermoautotrophicum, Methanosarcina acetivorans, Thermosynecoccus elongatus, and Chlorobium tepidum. The metabolic pathways of the last six translationally biased organisms are much less known than for those listed in Table 1. The analysis was also done for Helicobacter pylori (hpy) and Mycobacterium tuberculosis H37Rv (mtbRv), the second genome displaying some weak form of translational bias. All genomes have been completely sequenced. Genomes along with gene annotation were retrieved from the genomes directory of GenBank via FTP. All coding sequences (CDS) were considered, including those annotated as hypothetical and those predicted by computational methods only. An enzyme refers to a protein participating in a metabolic reaction, not a complex.

\section{Fast and Slow Growth}

An organism is fast growing if it has a doubling time of at most a couple of hours. Translational bias is usually detected in genomes of fast growers. Weak forms of translational bias can be detected for organisms that are not growing fast, for instance, $M$. tuberculosis $H 37 R v$, which has a doubling time of $20 \mathrm{~h}$, or $M$. thermoautotrophicum (Carbone et al. 2004).

\section{Metabolic Networks}

For all organisms in Table 1, H. pylori, and M. tuberculosisH37Rv, we used BioCyc software distribution, which includes EcoCyc and MetaCyc databases (Karp et al. 2000, 2002), version 7. The pathway/genome databases for B.subtilis, S. cerevisiae, M. tuberculosis, and $H$. influenzae were created by DoubleTwist Inc. The numbers of pathways and enzymatic reactions in Table 1 come from the BioCyc database. In BioCyc, pathways are organized into several functional classes, used in Figs. 1 and 3. We disregard from our analysis fragmentary pathways with no assigned function in the BioCyc network. The methane metabolism networks for organisms in Table 1 and Methanosarcina acetivorans and the photosynthetic networks (Calvin cycle, photosystems I and II) for Synechocistis are taken from the KEGG database at http://www.genome.ad.jp/kegg/ pathway.html.

\section{Translational Bias}

Translational selection refers to the benefit of an increased translational output for a fixed investment in the translational machinery (ribosomes, tRNA, elongation factors, etc.) if only a subset of codons (and their corresponding tRNAs) is used preferentially. Since the benefit of using a particular codon depends on how often it is translated, the strength of translational selection, and hence the degree of codon bias, is expected to vary with the expression level of a gene within an organism. This pattern has been confirmed for the organisms in Table 1 and others. Mutational bias (i.e., an excess or deficit of $\mathrm{G}+\mathrm{C}$ content compared to $\mathrm{A}+\mathrm{T}$ content, for instance) might obscure translational selection, which can appear in strong or weak forms (see below).

\section{Codon Bias, Codon Weights, and Codon Adaptation Index}

Sharp (Sharp and Li 1987) formulated the hypothesis that for each genome sequence $\mathrm{G}$, there is a set $\mathrm{S}$ of coding sequences, constituting roughly $1 \%$ of the genes in $\mathrm{G}$, which are representative of the dominating codon bias in $\mathrm{G}$. This bias can be described by listing a set of codon weights calculated on genes in S as follows: given an amino acid $j$, its synonymous codons might have different frequencies in $\mathrm{S}$; if $x_{i j}$ is the number of times that the codon $i$ for the amino acid $j$ occurs in $\mathrm{S}$, then one associates to $i$ a weight $w_{i j}$ relative to its sibling of maximal frequency $y_{j}$ in $\mathrm{S}$,

$$
w_{i, j}=x_{i, j} / y_{j}
$$


Such weights describe codon preferences in G and they are successfully used by Sharp to correlate gene expression levels with translational codon bias in fast-growing organisms. This is done by computing the CAI (Sharp and $\mathrm{Li}$ 1987) for all genes, $\operatorname{CAI}(g)=\left(\prod_{k=1}^{L} w_{k}\right)^{1 / L}$, where $g$ is a gene, $w_{k}$ is the weight of the $k$ th codon in $g, L$ is the number of codons in $g$, and $\mathrm{S}$ consists of genes coding for proteins known to be highly expressed, such as ribosomal and glycolytic proteins are for fast growers. Genes are then ranked by CAI values. Genes ranking the highest are the most biased and those ranking the lowest are the least affected by selective bias. For fast growers, genes with high CAI value turn out to be most expressed and translationally biased (Sharp and $\mathrm{Li}$ 1987).

More generally, CAI correlates with any kind of dominating bias in genomes (like GC content, preference for codons with $\mathrm{G}$ or $\mathrm{C}$ at the third nucleotide position, a leading strand richer in $\mathrm{G}+\mathrm{T}$ than a lagging strand), not just with translational bias (Carbone et al. 2004). This is shown by observing that the set of most biased genes $\mathrm{S}$ (consisting of $1 \%$ of the genes in $\mathrm{G}$ and where the size of $\mathrm{S}$ is suggested by Sharp's original work) can be automatically computed by a pure statistical analysis of the collection of all genes which is not based on biological knowledge of the organism. In the case of fast growers, $\mathrm{S}$ indeed is found to consist of highly expressed genes. The lack of reliance on biological knowledge allows computation of weights for organisms of unknown lifestyle. Codon weights, reference set $\mathrm{S}$, and CAI values are calculated with the program CAIJava written by the authors, which uses parsers of GenBank flat files from the Biojava (http://www.biojava.org) programming package. A description of the algorithm and a validation of the approach are reported by Carbone et al. (2004). The program CAIJava is available at $\mathrm{http}: / /$ www.ihes.fr/ carbone/ data.htm.

\section{Detection of Weak and Strong Forms of Translational Bias}

In Carbone et al. (2004), two numerical criteria were introduced to detect translational bias. The ribosomal criterion defines the $z$ score $(C A I(r)-\mu) / \sigma$, for each gene of a ribosomal protein $r$, where mean $\mu$ and standard deviation $\sigma$ are calculated for the CAI distribution over all CDS; this allows us to define the average $\bar{z}_{\text {Rib }}$ of $z$-scores for ribosomal proteins and say that an organism characterized by translational bias is expected to have high $\bar{z}_{\text {Rib }}$ i.e., $>1$. The strength criterion computes codon weights, as in (1), based on all genes in the genome $\mathrm{G}\left(w_{k}(G)\right)$ and on the genes in the set $\mathrm{S}\left(w_{k}\right)$ and expects the difference between $w_{k}(G)$ and $w_{k}$ to be large for translationally biased genomes (i.e., $\sum_{k=1}^{64}\left(w_{k}(G)-w_{k}\right) / 2>8$; this sum is an indicator of the number of amino acids having different preferred codons in the entire genome and in the set of most biased genes; the threshold 8 has been empirically calculated on known translationally biased organisms [Carbone et al. 2004]). The combination of the two criteria allows determination of which genomes are strongly translationally biased, that is, those satisfying both criteria, from those that are weakly so, that is, those that only satisfy the ribosomal criterion. Notice that our numerical criteria provide quantitative values ranging within a continuous interval and that, based on these values, one can identify strong, weak, and absent forms of bias as well as finer classifications.

\section{Codon Bias Signatures}

Given a genome sequence, weak and strong tendencies toward content bias, translational bias, and strand bias can be identified (Carbone et al. 2004). The collection of strong codon biases affecting CDS coding is referred to as the codon bias signature of the genome. Signatures for the genomes in Table 1 have been analyzed by Carbone et al. (2004). Codon signatures are indicators of the evolutionary pressure organisms undergo.

\section{Ranking of Pathways in the Metabolic Network of an Organism}

For each pathway $\mathrm{P}$ of the metabolic network $\mathrm{M}$ of an organism, we compute the mean of the CAI values of the enzymes involved in $\mathrm{P}$. If an enzyme is involved in more than one reaction in P, its CAI value is counted with multiplicity. Some enzymes in a pathway might contribute no CAI value due to the fact that they have not been identified in the genome. This numerical index of pathways is called the Pathway Index (PI), and we denote PI(P) the mean value for a pathway $\mathrm{P}$. This index allows us to define a ranking of pathways involved in metabolism.

There is a significant difference in PI values between pathways (see Table 1). S. cerevisiae is the organism in Table 1 that displays the most variation of PI values among pathways, with $\mu_{M}=0.31$ and $\sigma_{M}=0.12$, where $\mu_{M}$ and $\sigma_{M}$ are the mean and the standard deviation of the distribution of $\mathrm{PI}(\mathrm{P})$ values for $\mathrm{P}$ in $\mathrm{M}$, with a minimum and a maximum PI value of 0.15 and 0.73 for the methionine biosynthesis from homoserine pathway and the glycolysis pathway. This large spread among PI values justifies the use of the average CAI of genes involved in a pathway as a biological relevant measure. In the sequel, we consider the inter$\operatorname{val}\left[\mu_{M}-\sigma_{M}, \mu_{M}+\sigma_{M}\right]$, and intuitively, we say that $\mathrm{PI}(\mathrm{P})$ values $\geq \mu_{M}+\sigma_{M}$ are "high" and those $\leq \mu_{M}-\sigma_{M}$ are "low."

\section{Clustering of Genes Along the Genome and Pathway Index}

The pathway index is independent of the localization of genes along the genome. To see this, we consider the enzyme positions along the genomic sequence, and we allow up to three genes coding for proteins that do not belong to the pathway to intercalate between two genes coding for enzymes in the pathway.

We say that two enzymes are clustered if they are located in this pattern along the genomic sequence. The results are displayed in Table 1. Most pathways consist of enzymes which are not clustered in the same genomic region. On average, only $7-8 \%$ of enzymatic reactions are involved in "clustered" pathways. In particular, in E.coli this value drops to $3 \%$.

\section{Ranking of Metabolic Networks and Network Comparison Across Organisms}

To compare the distribution of codon bias in metabolic pathways across organisms, we define the Relative Pathway Index as $R P I(P)=\left(P I(P)-\mu_{M}\right) / \sigma_{M}$, where the mean and the standard deviation are taken over all Ps in the metabolic network $M$ of an organism. This measure is used to compare organisms with respect to common metabolic pathways. After this normalization of PI values we can define the Comparative Pathway Index $\mathrm{CPI}(\mathrm{P})$ to be the average of the RPI(P) for all organisms sharing a pathway $\mathrm{P}$, for comparison of the ranking of pathways over multiple organisms.

For visual representation and comparison (see Fig. 1), we recall that an $\mathrm{RPI}(\mathrm{P})$ value of +1 corresponds to a pathway with a bias one $\sigma$ above the mean for the organism, and a value of -1 , one $\sigma$ below. So the interval $[-1,1]$ is mapped into a continuous range of colors, going gradually from violet, blue, and green (lower values) to yellow, orange, and red (higher values) and pathways are as- 


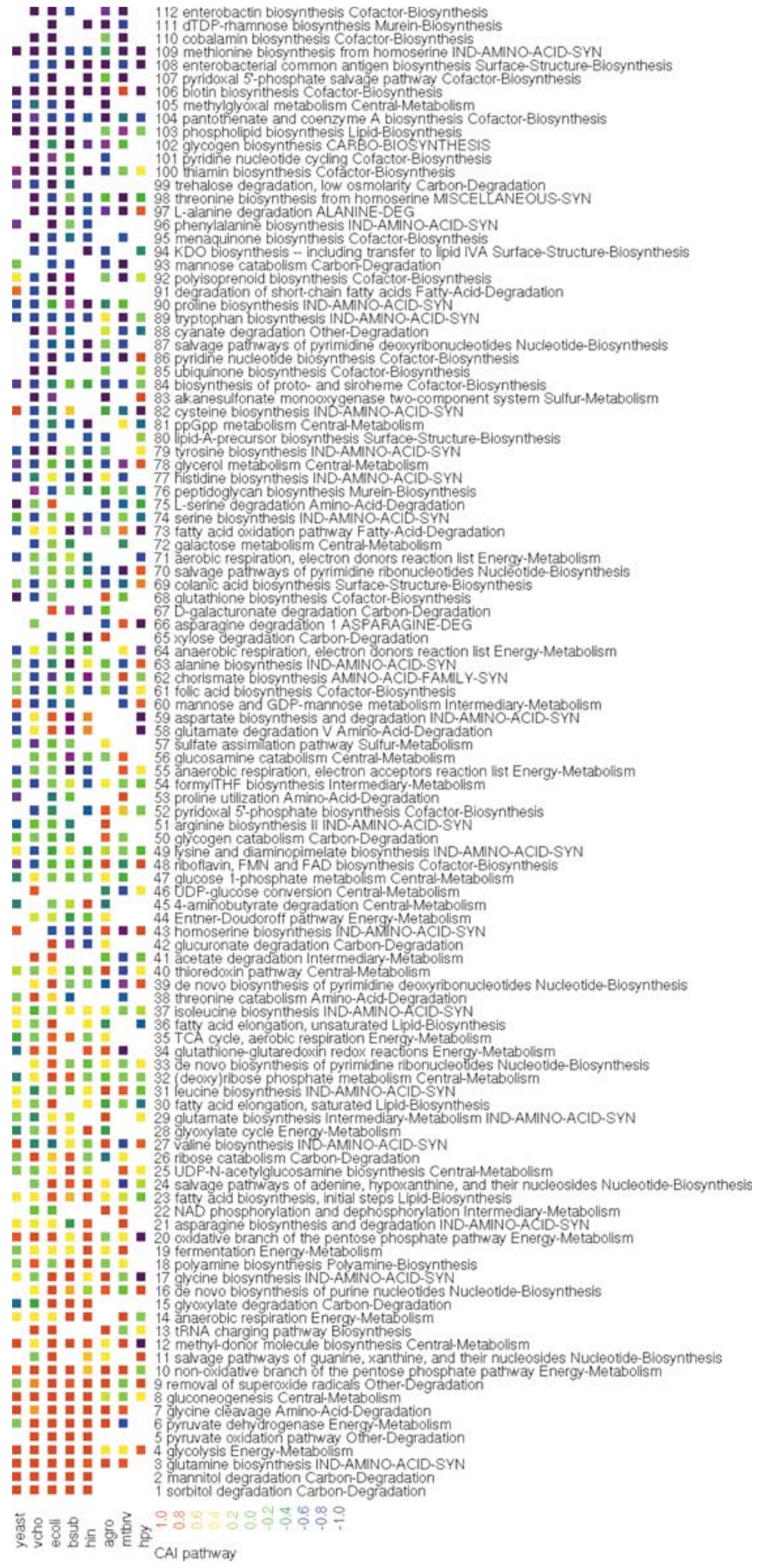

Fig. 1. Metabolic pathways occurring in four or more organisms among those listed in Table 1, Mycobacterium tuberculosis H37Rv (mtbrv), and Helicobacter pylori (hpy). For each organism, the RPI value associated with a pathway, if it exists, is indicated by the corresponding ranking color. Each row corresponds to a pathway denoted by (Biocyc) name and functional classification. Pathways are increasingly ordered from top to bottom by CPI. signed the corresponding color. Pathways with RPI(P) values falling outside the interval take the values of the closer extremes, -1 (violet), +1 (red). The mapping provides a good spread of colors for a suitable reading of metabolic differences, especially for CAI homogeneous organisms, independent of the width of the underlying statistical distribution.

\section{Transcriptomic Data and Expression Levels}

Transcriptomic data for $S$. cerevisiae are taken from the study reported by Holstege et al. (1998) and based on Affymetrix GeneChip high-density oligonucleotide array (HDA) technology (downloaded from http://www.wi.mit.edu/young/expression.html in 1999, and 
Table 2. PI of methane metabolism pathway (PI[Met]) in some translationally biased organisms

\begin{tabular}{llllll}
\hline Organism & $\mu$ & $\sigma$ & $\mu+\sigma$ & PI(Met) & $\mu_{R}$ \\
\hline Agrobacterium tumefaciens & 0.44 & 0.11 & 0.55 & 0.51 & 0.64 \\
Bacillus subtilis & 0.37 & 0.07 & 0.44 & 0.48 & 0.64 \\
Escherichia coli & 0.30 & 0.10 & 0.40 & 0.48 & 0.60 \\
Haemophilus influenzae & 0.38 & 0.09 & 0.47 & 0.36 & 0.58 \\
Vibrio cholerae & 0.28 & 0.08 & 0.36 & 0.18 & 0.64 \\
Saccharomyces cerevisiae & 0.16 & 0.12 & 0.28 & 0.57 & 0.63 \\
Methanosarcina acetivorans & 0.50 & 0.06 & 0.56 & & \\
\hline
\end{tabular}

Note. Only M. acetivorans is known to use methane metabolism in an essential way.

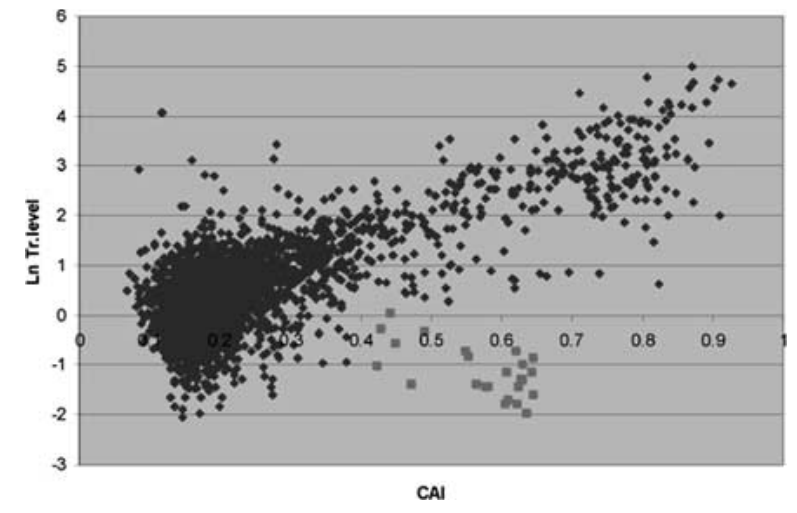

Fig. 2. Transcriptional level of S. cerevisiae genes are plotted (in $\log$ scale) with CAI values; a group of outliers displaying high CAI values and low transcriptional levels is illustrated as light gray squares.

available at http://www.ihes.fr/ carbone/data.htm). It concerns a set of 4849 genes whose expression profiles are determined by growing yeast cultures to midlog phase. Expression levels are defined as the numbers of copies of a given mRNA per yeast cell. The arrays can detect as few as $0.1 \mathrm{mRNA}$ molecule/cell; the dynamic range over which detection is accurate is approximately $0.1-100$ mRNA molecule/cell. The yeast genome is covered by four HDAs and each gene is represented on the HDA by 20- to 25-mer oligos that match the sequence of the message (perfect match oligos) and 20 oligos that are identical but differ by one base (mismatch oligos). Expression levels are calculated by subtracting the signal of a mismatch from its perfect match partner and averaging the difference for each oligo pair for a given gene. The average difference value is a measure of the expression level of that gene used in Fig. 2.

Transcriptomic data on the $S$. cerevisiae cell cycle under diauxic shift are taken from deRisi et al. (1997). Expression levels are computed as absolute fluorescence intensity minus background. Because of the considerable variation across microarray experiments, expression levels shown do not translate into absolute mRNA concentrations and are informative only when taken in relation to other genes. Despite the differences in technology and definitions of expression levels, transcriptomic data collected at different time points by deRisi et al. (1997), when plotted with CAI values (not shown), display shapes similar to Fig. 2, constructed from Holstege et al. (1998). Compare with the slope of the distributions of pathways in Fig. 3.

\section{Transcriptomic Data as Indices of Metabolic Networks}

A transcriptional pathway index for a metabolic network can be calculated from mRNA abundance levels obtained through microarray analysis. We define the $\mathrm{PI}_{\mathrm{T}}(\mathrm{P})$ of a pathway $\mathrm{P}$ as the average of the expression levels of the enzymes involved in $\mathrm{P}$ at a given environmental condition (analogous to our definition of an index based on CAI values). Again, enzymes are counted with multiplicity and omitted where data are lacking.

Normalization of $\mathrm{PI}_{\mathrm{T}}$ values is done as for PIs above, by defining $\mathrm{RPI}_{\mathrm{T}}(\mathrm{P})=\left(\mathrm{PI}_{\mathrm{T}}(\mathrm{P})-\mu_{T}\right) / \sigma_{T}$, where $\mu_{T}, \sigma_{T}$ are the mean and standard deviation of the distribution of $\mathrm{PI}_{\mathrm{T}}(\mathrm{P})$ values for $\mathrm{P}$ in $\mathrm{M}$ at a given time point. The visual representation (in Fig. 3) of the interval $[-1,+1]$ is mapped into a continuous range of colors, going gradually from violet to red, as above. Pathways $\mathrm{P}$ with $\mathrm{RPI}_{\mathrm{T}}(\mathrm{P})$ value falling outside the interval take the values of the closer extremes (violet or red).

For a given organism and a set of microarray data experiments obtained under different biological conditions, we define the Evolutionary Pathway Index (EPI) to be a numerical ranking of pathways $\mathrm{P}$, ordering them by the maximum among the $\mathrm{PI}_{\mathrm{T}}(\mathrm{P})$ values within all conditions in the set. Intuitively, the maximum value obtained over a sufficiently large set of transcriptomic data corresponding to different biological states should provide information on those pathways and enzymes that are rarely highly translated but that on certain occasions need to be expressed in large quantities and/or rapidly.

An evolutionarily meaningful set of experiments is a set of transcriptomic data which presents a sufficiently large variation of gene expression levels under sufficiently large changes in biological conditions. The transcriptomic data on the diauxic shift of deRisi et al. (1997) present such variation.

\section{Metabolic Activities Derived from CAI Values}

Genes with high CAI values in fast-growing organisms are commonly interpreted as those which are involved in maintaining this growth rate or in enzymatic activities with a rapid response (Gouy and Gautier 1982; Sharp and Li 1987; Sharp et al. 1988; Médigue et al. 1991; Shields and Sharp 1987; Carbone et al. 2004). Genes with such properties are coding for ribosomal proteins, heat shock proteins, antioxidant proteins, proteins involved in the respiratory chain, structural genes (for eukaryotes), and enzymes involved in the metabolic pathways of amino acids biosynthesis. Functional groups of genes which have low CAI values are transcription factors, genes involved in pattern formation (for eukaryotes), cell cycle progression, clock genes, genes involved in the metabolic pathways of nucleotide biosynthesis, carbohydrates, lipids, and secondary metabolites, but also degradation as the 

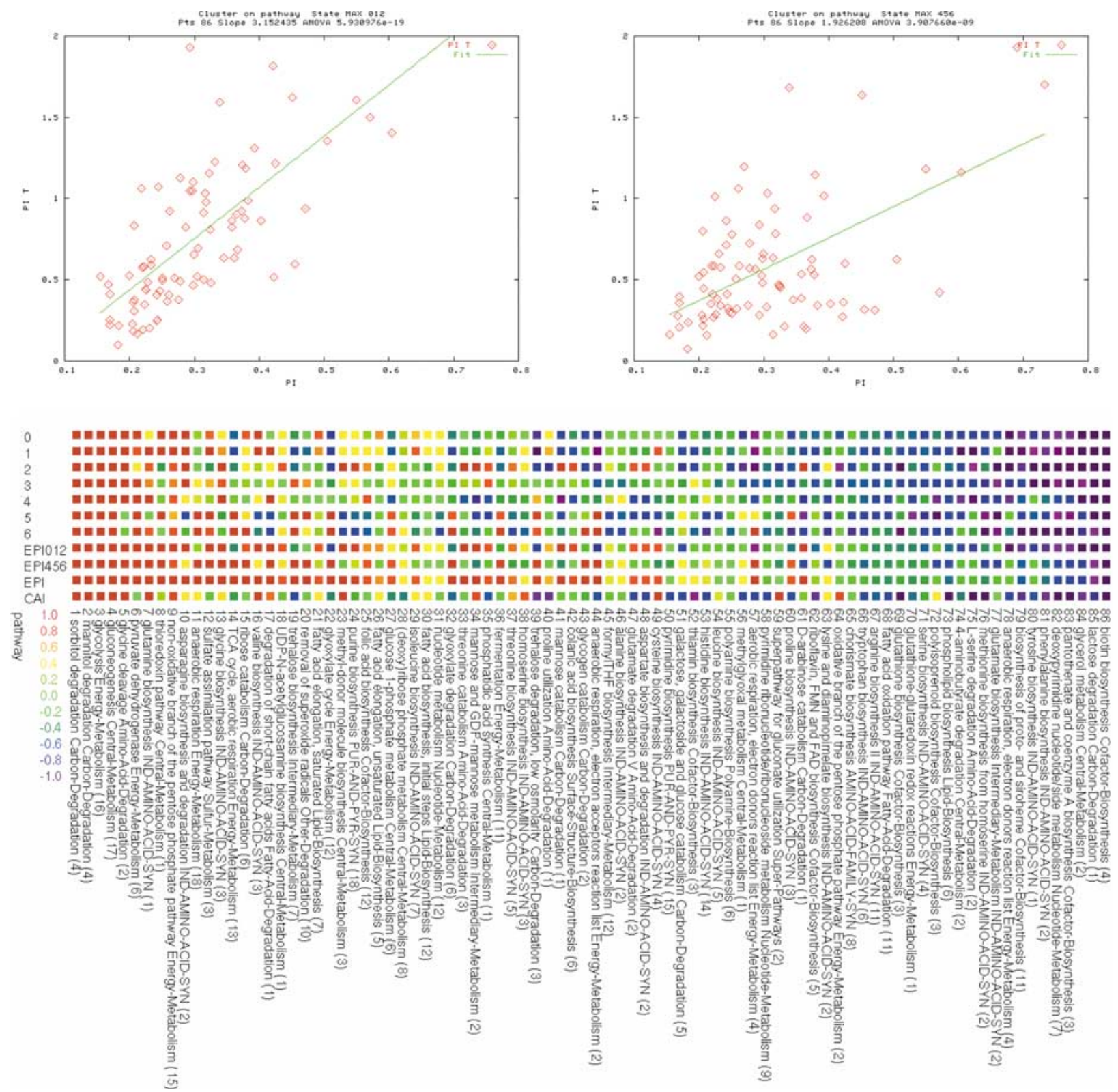

Fig. 3. S. cerevisiae cell cycle during diauxic shift. Top: Plots corresponding to the activity of 86 metabolic pathways during time points $0,1,2$ (left) and 4, 5, 6 (right). Each point in a plot is a metabolic pathway $\mathrm{P}$ represented by its maximum $\mathrm{PI}_{\mathrm{T}}(\mathrm{P})$ ( $y$-axis) value calculated over three time points and $\mathrm{PI}(\mathrm{P})$ ( $x$-axis) value; slopes of the distribution of metabolic pathways go down from the anaerobic state (fermentation), left to the aerobic state (respira-

ubiquitin-proteosome pathway, or apoptosis. When the organism is not growing fast, then high-CAIvalue genes are often correlated with a dominant bias of other origins, like GC3 content or strand bias (Carbone et al. 2003).

A more careful analysis reveals that for fast growers, there exists a large gap between the mean $(\mu)$ of the CAI distribution over all CDSs and the average CAI value for ribosomal proteins $\left(\mu_{R}\right)$; for E. coli, for instance, $\mu_{R}=0.60$ and $\mu=0.30$, with a stan- tion), right. (Also, the $r^{2}$ values of the fits are 0.782 and 0.583 , respectively, based on the least-squares fit model). Bottom: RPI value of metabolic pathways during time points $0 \ldots 6$ of the diauxic cycle; EPI value for time points 0, 1, 2 (EPI012), 4, 5, 6 (EPI456), and all (EPI); and RPI(P) values (CAI). Pathways (cited by name, functional classification, and number of distinct enzymes involved) are ordered by EPI. dard deviation $\sigma=0.10$. For many species that do not grow fast, the gap between $\mu$ and $\mu_{R}$ is less important, but ribosomal proteins still display a relatively high CAI, that is $\mu_{R}>\mu+\sigma$. Mycoplasma pulmonis, for instance, has $\mu_{R}=0.7, \mu=0.57$, and $\sigma=0.05$. This organism has a dominant codon bias with no apparent translational origin but, rather, a dominant AT3 bias (Carbone et al. 2004); nevertheless, the codon composition of its ribosomal proteins is largely affected by AT3 bias. 
For those translationally biased organisms for which not much is known of the metabolic activity of their enzymes, CAI analysis of specific genes and comparison with the coding of ribosomal proteins suggest a criterion to infer lifestyle: genes whose CAI value is close to $\mu_{R}$, that is, $\geq \mu_{R}-\sigma$, can be treated as indicators of important metabolic activities for the organism. (Notice that this bound implicitly considers the "strength" of the translational bias of the genome, indicated by how large the interval $\mu_{R}-\mu$ is.) We discuss this hypothesis for ferrodoxin metabolism, photosynthesis, and methanogenesis.

\section{Ferrodoxin in P. abissi}

The archaea $P$. abissi has been computationally classified as a translationally biased organism (Carbone et al. 2004), with $\mu=0.45, \sigma=0.07$, and $\mu_{R}=0.59$. Among its most biased genes, besides ribosomal proteins and elongation factors, we find ferrodoxin $(\mathrm{CAI}(\mathrm{fdxA})=0.71), \quad$ ferredoxin oxidoreductase $(\mathrm{CAI}($ for $)=0.63)$, and keto-valine-ferredoxin oxidoreductase $\gamma$-chain $(\mathrm{CAI}(\mathrm{PAB} 1470)=0.62)$. Ferredoxin appears to be the major metabolic electron carrier in pyrococci (Cohen et al. 2003). After reduction during peptide or sugar degradation, it is mainly reoxidized by a membrane-bound hydrogenase (Silva et al. 2000), potentially generating membrane potential. In addition, ferredoxin has been suggested to be reoxidized by ferredoxin-NADP oxidoreductase (Silva et al. 2000; Schut et al. 2001). Moreover, NADPH can also be oxidized via the conversion of pyruvate to alanine, via glutamate dehydrogenase $(\mathrm{CAI}(\mathrm{PAB} 0391)=0.74)$ and alanine aminotransferase $(\mathrm{CAI}(\mathrm{PAB} 1810)=0.55)$ (Ward et al. 2000). The high bias of all genes involved in ferrodoxin metabolism hints at the importance of this pathway for the organism.

\section{Photosynthesis Pathways: Synechocystis}

The cyanobacterium Synechocystis is classified to be translationally biased (Mrázek et al. 2001) and its most biased genes are ribosomal proteins, phycocyanin $(\mathrm{CAI}(\mathrm{cpcB})=0.79, \mathrm{CAI}(\mathrm{cpcA})=0.78)$, allophycocya$\operatorname{nin}(\mathrm{CAI}(\mathrm{apcB})=0.78, \mathrm{CAI}(\mathrm{apcA})=0.72)$, photosystem II proteins $(\mathrm{CAI}(\mathrm{psbA} 2)=\mathrm{CAI}(\mathrm{psbA} 3)=0.77$, $\mathrm{CAI}(\mathrm{psbI})=0.70)), \quad$ fructose-1,6-bisphosphatealdolase $(\mathrm{CAI}(\mathrm{cbbA})=0.71)$ and ferredoxin $(\mathrm{CAI}($ petF $)=0.76)($ Carbone et al. 2004). The presence of proteins involved in photosynthesis within the most biased genes is a good indicator of the known photosynthetic activity and lifestyle of Synechocystis. In fact, the metabolic networks of photosystem I, photosystem II, and the Calvin cycle have PI $=0.62$, 0.58 , and 0.62 , respectively, and from $\mu_{R}=0.60$, $\mu=0.50$, and $\sigma=0.07$, a photosynthetic preference of Synechocystis is confirmed with $\mathrm{PI}(\mathrm{P}) \geq \mu_{R}-\sigma$, for all three pathways $\mathrm{P}$ above.

\section{Methane Metabolism: Methanosarcina acetivorans}

The archaeon $M$. acetivorans has been computationally classified as a translationally biased organism (Carbone et al. 2004), with $\mu_{R}=0.63, \mu=0.50$, and $\sigma=0.06$. Besides ribosomal proteins, methanol-5 hydroxybenzimidazolylcobamide comethyltransferase $(\mathrm{CAI}(\mathrm{mtaB} 1)=0.83, \mathrm{CAI}(\mathrm{mtaB} 2)=0.75$, $\mathrm{CAI}(\mathrm{mtaC} 1)=0.71, \quad \mathrm{CAI}(\mathrm{mtaB} 3)=0.68), \quad$ methyl coenzyme M reductase $(\mathrm{CAI}(\mathrm{MA} 4546)=0.79$, $\mathrm{CAI}(\mathrm{MA} 4547)=0.76, \quad \mathrm{CAI}(\mathrm{MA} 4550)=0.73), \quad$ and methylcobamide methyltransferase isozyme $M$ $(\mathrm{CAI}(\mathrm{mtaA})=0.72)$ are among the most biased genes. In particular, $\mathrm{PI}(\mathrm{Met})=0.57 \geq \mu_{R}-\sigma$, where Met is the methane metabolism network. As shown in Table 2, no organism in Table 1 has PI(Met) within $1 \mathrm{SD} \sigma$ from $\mu_{R}$, while $M$. acetivorans does. From this and the high CAI value of proteins involved in methane metabolism, one can infer the unusual living environmental conditions of these bacteria.

\section{Metabolic Activities and RPI Values: Analysis of Metabolic Networks Across Species}

For translationally biased organisms whose metabolic network has been partially reconstructed, CAI values of enzymes might be profitably used as indicators of preferential metabolic pathways and of lifestyle. Using the notion of Relative Pathway Index (RPI; defined in Materials and Methods), we observe that pathways can be grouped into classes with low, medium, and high RPI. As shown in Fig. 1 (and the same conclusions hold for pathways shared by fewer than four organisms), there are pathways that display the same bias across organisms: shared high RPI values (red and orange squares) suggest that a metabolic activity is favored and that it has a possibly constitutive regime; shared low RPI values (violet and blue squares) suggest that a metabolic activity is likely not involved in chains of rapid enzymatic responses. Pathways with low RPI values are essentially cofactors-coenzymes involved in vitamin biosynthesis which are known not to be usually produced with a high efficiency, and pathways with high RPI values are mainly involved in energy metabolism. There is a third group of pathways that display mixed RPI values across species, and from this set of pathways one might expect to infer differences in lifestyle. These pathways are mainly involved in central metabolism and amino acid synthesis and degradation. A thorough organism comparison can be realized by fol- 
lowing the ranking described by the metabolic maps in Fig. 1. Here we consider a few specific pathways which are highly biased in one organism, but not in others, and argue, based on experimental evidence, in favor of their importance for the life of the organism. Below, a pathway is associated with the numbering listed in Fig. 1.

\section{Glycolytic Pathway}

For all translationally biased organisms in Table 1, the glycolytic pathway is expected to be especially favored because of fast growth. It has been observed that most genes with the highest CAI values are involved in this pathway (Sharp and Li 1987), and accordingly, we find that the RPI value of the pathway (4) is one of the highest as displayed in Fig. 1.

\section{Glutamate Biosynthesis for A. tumefaciens}

The genome of A. tumefaciens contains seven glutamine synthetase genes encoded in three distinguished types $($ with $\mathrm{CAI}(\mathrm{g} \ln \mathrm{A} \mathrm{I})=0.77, \mathrm{CAI}(\mathrm{g} \ln \mathrm{A} \mathrm{II})=0.74$, $\mathrm{CAI}(\mathrm{g} \ln \mathrm{A}$ III $)=0.57)$, and the presence of multiple copies of these genes seems to be related to the observation that this bacterium requires high concentrations of glutamate for optimal growth (Wood et al. 2001). The RPI value of the glutamate biosynthesis pathway (29) in A. tumefaciens is high; note that for all other organisms in Table 1 this pathway has a much lower RPI.

\section{Metabolic Differences Between S. cerevisiae and Aerobic Bacteria}

A number of pathways involved in amino acid biosynthesis (homoserine [43], valine [27], and cysteine [82]) and intermediate metabolism (mannose and GDP-mannose [60]) are highly biased in S. cerevisiae but not in most translationally biased aerobic bacteria in Table 1 . The highly biased mannose and GDP-mannose metabolic pathway confirms the experimental evidence that $S$. cerevisiae can produce ethanol from glucose and mannose if the concentrations of sugars are high or when the yeast is grown under anaerobic conditions (Ratledge 1991), and this finding agrees with the statistical evidence reported in the next section, that the genome of $S$. cerevisiae has undergone strong selective pressure favoring a predominantly fermentative metabolism.

Also, the pyruvate dehydrogenase pathway (6) and the removal of superoxide radicals pathway (9) are highly biased in aerobic bacteria in Table 1 but not in the $S$. cerevisiae genome. In this respect, note that aerobic conditions lead to the generation of acetyl-CoA and pyruvate dehydrogenase, and its coenzymes play a decisive part in this reaction. Also, since oxygen and its derivatives are toxic and can lethally damage certain cellular components, protective enzyme systems have been evolved by aerobic organisms (that use oxygen as terminal electron acceptor in respiration in a crucial way), and this agrees with the removal of superoxide radical pathway being highly biased. On the other hand, again, the absence of bias for these two pathways found in $S$. cerevisiae sustains the hypothesis of selective pressure favoring fermentation in this organism (see below).

\section{L-Serine Degradation in E. coli}

L-serine is a preferred growth factor for $E$. coli and experimental evidence is reported in several studies (Pizer and Potochny 1964). The high RPI value of the L-serine degradation pathway (75) justifies the sensitivity of E. coli to L-serine and its potential toxicity to the cell (Newman and Walker 1982).

\section{Ammonia Assimilation Pathway in E. coli}

The enteric bacterium E. coli (and many other organisms) have two primary pathways of glutamate synthesis, the glutamate dehydrogenase (GDH) and the glutamine synthetase-glutamate synthase (GOGAT) pathway. GDH plays a role in glutamate synthesis when $E$. coli is under energy (and carbon) restriction but not under ammonium or phosphate restriction, and the GOGAT pathway is responsible for glutamate synthesis when energy is plentiful or when the ammonium or phosphate concentration becomes low. In an energy-rich (glucose-containing), nitrogen-poor environment, glutamine synthetase and glutamate synthase form the ammonia assimilatory cycle GOGAT, which is ATP-dependent and essential for nitrogenlimited growth and for steady-state growth with some sources of nitrogen. We found that the RPI value of GOGAT is high (with $\mathrm{CAI}(\mathrm{g} \ln \mathrm{A})=0.60$, $\mathrm{CAI}(\mathrm{gltD})=0.49$, and $\mathrm{CAI}(\mathrm{gltB})=0.42)$, and this is in agreement with the essential role of the pathway. In particular, RPI(GOGAT) is higher than RPI(GDH) (with $\mathrm{CAI}(\mathrm{gdhA})=0.41)$, and this sustains the experimental evidence that GOGAT plays at least two roles for which GDH cannot substitute. GOGAT can fix ammonium into organic molecules (glutamine, thence glutamate and other compounds) when the external concentration of ammonium is low, and it reduces the concentration of glutamine when that concentration becomes high (Reitzer 1986). Note also that a strain deficient in glutamate dehydrogenase has no observable growth phenotype under usual growth conditions (Reitzer 1986) but that GDH appears to be important during energy-limited growth because, 
through its use, the cost of biosynthesis is scaled down (Helling 1994).

\section{Evolution of Metabolic Networks and Transcriptomic Data in S. cerevisiae}

The high CAI value of genes is a good indicator not only of the high level at which a protein might be constitutively translated but also of the "possibility" for a protein to be highly expressed under very specific biological conditions. The next two examples suggest that codon bias is more informative than expression data for those enzymes involved in the wild that may not be highly expressed in laboratory experiments.

\section{Seripauperine Proteins in Yeast}

We consider the set of outlier ORFs (see Fig. 2 and also Carbone et al. [2003]) in the correlation plot between S. cerevisiae transcriptomic data (Holstege et al. 1998) and CAI values (Carbone et al. 2003). These outliers correspond to the gene family of seripauperines (the majority of unknown ORFs within the set are homologous to PAU1), which are small proteins (100 aa) presenting a strong sequence identity to proteins that are serine rich but deprived of the serinerich domains. These genes are highly induced during fermentation conditions or long-lasting anaerobism (experimental evidence is reported on brewery yeast by James et al. [2003]), while their expression is difficult to detect under normal conditions. (Viswanathau et al. 1994)

\section{Hem13 Proteins in Yeast}

Hem13 is an enzyme involved in the heme biosynthetic pathway of $S$. cerevisiae. It is an oxidase with oxygen as a substrate (Zitomer and Lowry 1992). It displays a quite high CAI value (0.47), compared to $\mu=0.16$ and $\sigma=0.12$ for all $S$. cerevisiae genes, and it is highly translated under anaerobic conditions (Amillet et al. 1995) but not otherwise. Several facultative aerobic organisms contain a second enzyme catalyzing the same reaction as Hem 13 but where the role of oxygen is replaced by molybdenum cofactor and S-adenosylmethionine. Since S. cerevisiae does not contain such an alternative enzyme, it is plausible that during anaerobism (or micro-aerobism) $S$. cerevisiae produces a large quantity of $\mathrm{Hem} 13$ to efficiently use the little oxygen present in the medium and to induce the heme necessary for its metabolism.

These examples suggest that we look at the drastic changes in the expression of genes involved in fundamental cellular processes which are detectable along the switch from anaerobic growth (fermentation) to aerobic respiration upon depletion of glucose in the cell cycle of $S$. cerevisiae. This cycle is known as the diauxic shift; it has been documented by Johnston and Carlson (1992) and studied through DNA microarrays by deRisi et al. (1997). Through this global view of the way the cell adapts to a changing environment, and by comparing transcriptomic levels to PI values of metabolic networks, we aim to detect those pathways that might be important for cell viability but whose relevance might be difficult to observe in laboratory experiments. For this, we analyze thoroughly the transcriptomic data of deRisi et al. (1997) collected along seven time points (0...6) at successive 2-h intervals during diauxic shift. We look at slopes of the regression lines for $\mathrm{PI}_{\mathrm{T}}$ values in terms of PI values of metabolic pathways and notice that the change in transcriptomic values taking place from fermentation (corresponding to time points $0,1,2$ ) to aerobic respiration (corresponding to time points 4 , $5,6)$ globally affects the entire set of metabolic pathways involved in the cell cycle. The regression lines associated with the successive time points $0 . .66$, corresponding to the progressive depletion of glucose in the medium, display gradually decreasing slopes (not shown). In particular, pathways with a low PI value (that is, $\mathrm{PI}(\mathrm{P})<0.25$ ) on time points $0,1,2$ suddenly augment their $\mathrm{PI}_{\mathrm{T}}$ on time points $4,5,6$, and accordingly, pathways with a medium-high PI value $(0.25<\mathrm{PI}(\mathrm{P})<0.45)$ decrease in $\mathrm{PI}_{\mathrm{T}}$. (Figure 3 summarizes this observation; it shows average $\mathrm{PI}(\mathrm{P})$ and $\mathrm{PI}_{\mathrm{T}}(\mathrm{P})$ values computed over time points $0,1,2$ and 4, 5, 6 and decreasing slopes for the corresponding regression lines.) The list of affected pathways is given in Fig. 3. Below, we refer to pathways with the numbering listed in Fig. 3.

It has been suggested that the high slope associated with the fermentative state (i.e., a strong correlation between CAI values and expression levels) might indicate a strong evolutionary pressure that favored a predominantly fermentative metabolism of yeast in the wild (Wagner 2000). Moving the organism to an unfavored state is then reflected by the drop in slope as less often used genes (with correspondingly lower CAI values) are moved to higher expression levels. Our analysis confirms that pathways which are highly active during fermentation are also highly biased and leads us to conclude that the coding sequences of enzymes involved in specific metabolic functions (indexed by PI values) describe the physiological responses of yeast precisely during fermentation (indexed by $\mathrm{PI}_{\mathrm{T}}$ ). The examples of seripauperine and Hem 13 proteins discussed above, presenting strong codon bias (i.e., large CAI values) and large RNA abundance during fermentation, sustain this hypothesis. Other metabolic pathways playing a crucial role in fermentation are detectable from the 
analysis of the diauxic cycle. We see that pathways with a high RPI value (red and orange squares in the CAI line in Fig. 3, bottom) and at least two enzymes participating in the metabolic reaction have a high EPI value during fermentation (i.e., at time points 0 , 1, 2; see red and orange squares in the EPI012 line in Fig. 3). They are glycolysis (3), gluconeogenesis (4), glycine cleavage (5), glutamine biosynthesis (7), sorbitol (1) and mannitol (2) degradation, and the nonoxidative branch of the pentose phosphate pathway (9). These are also highly expressed during aerobic respiration, while mannose, GDP-mannose (34), and valine biosynthesis (16), methyl-donor molecule biosynthesis (23), and cysteine biosynthesis (49) are highly expressed only during fermentation. There are three pathways where this claim is not verified by the diauxic cycle dataset (i.e., RPI is high but EPI012 is low): homoserine biosynthesis (38), gluconate utilization (59), and the oxidative branch of the pentose phosphate pathway (64). There is experimental evidence, however, of the involvement of the oxidative pentose phosphate pathway and of the gluconate utilization pathway during fermentation. It has been reported (Middelhoven et al. 2000) that under anaerobic conditions, the yeast Saccharomyces bulderi rapidly ferments ( $\delta$-gluconolactone to ethanol and carbon dioxide. In particular, levels of the pentose phosphate pathway enzymes were 10-fold higher in $\delta$-gluconolactone-grown anaerobic cultures than in glucose-grown cultures (van Dijken et al. 2002). Also, growth of $S$. cerevisiae on $\delta$-gluconolactone was found to be associated with a specific coordinate induction of the synthesis of two enzymes of the oxidative pentose phosphate pathway, 6-phosphogluconate dehydrogenase and 6-phosphogluconolactonase, together with that of gluconokinase (Sinha and Maitra 1992). For the homoserine biosynthesis pathway we could not find experimental evidence of its involvement in fermentation, and we claim that there is some time point during fermentation when genes belonging to this pathway are highly expressed.

\section{Discussion}

New measures aiming to numerically study biological and evolutionary questions and to index metabolic pathways across translationally biased organisms with respect to genetic coding are introduced. The approach seems to apply profitably also to genomes displaying weak forms of translational bias. Even though the interval between $\mu$ and $\mu_{R}$ is much less pronounced in genomes that present weak forms of translational bias, CAI analysis and RPI analysis help to determine metabolic preferences and to reveal interesting information on lifestyle. We discuss two examples.
The Case of $\mathrm{M}$. thermoautotrophicum

The archaeon $M$. thermoautotrophicum has been computationally classified to have a weak form of translational bias (Carbone et al. 2004), with $\mu=0.51, \sigma=0.07$, and $\mu_{R}=0.59$. Besides ribosomal proteins, tungsten formylmethanofuran dehydrogenase and methyl reductase dehydrogenase, that is, genes involved in methane metabolism, are among the most biased genes in Methanobacterium.

\section{The Case of M. tuberculosis H37Rv}

The virulent strain $M$. tuberculosis $H 37 R v$ has a doubling time of about $20 \mathrm{~h}$ and its genome presents a weak form of translational bias (Carbone et al. 2004), with $\mu_{R}=0.57, \mu=0.50$, and $\sigma=0.07$. The metabolic map of $M$. tuberculosis H37Rv is displayed in Fig. 1. Among networks with a high RPI value, a key example is provided by the biotin biosynthesis pathway (106 in Fig. 1), which turns out to have a very high RPI for Mycobacteria (note that also the clinical isolate $M$. tuberculosis CDC displays a very high RPI value for biotin biosynthesis; not shown) but a very low RPI for all other organisms we studied: $M$. tuberculosis has a lipidrich cell envelope which contributes to virulence and antibiotic resistance. Acyl-coenzyme A carboxylase, which catalyzes the first committed step of lipid biosynthesis, consists in mycobacteria of two subunits, one of which is indeed biotinylated. Genes encoding a biotinylated protein have been cloned and sequenced and the presence of biotin-binding sites has been experimentally demonstrated (Norman et al. 1994). Several other metabolic pathways have been ranked high in RPI for $M$. tuberculosis $H 37 R v$ despite their low RPI values for other species. Experiments demonstrated that the chorismate biosynthesis pathway (62) is essential for the viability of M. tuberculosis H37Rv (Parish and Stoker 2002) and that the aspargine degradation pathway (66), pyridoxal 5-phosphate biosynthesis (52), valine degradation (not shown in Fig. 1), and leucine biosynthesis (31) are also essential pathways (Sassetti et al. 2003). Finally, the ppGpp metabolic pathway (81) is essential for long-term survival of mycobacteria under starvation conditions (Primm et al. 2000). All these pathways of M. tuberculosis have high RPI values. Note that the serine biosynthesis pathway, which has been detected to be essential by Sassetti et al. (2003), has been found to be very highly ranked in the $M$. tuberculosis CDC strain (not shown).

In genomes where codon usage has a strictly mutational origin (i.e., strand bias or compositional 
bias), one expects highly ranked pathways to have a different base composition with no associated biological meaning. However, there are genomes that might present weak tendencies toward translational bias which can be identified by our approach but cannot be detected with classical statistical methods such as hidden Markov chains and multivariance statistical methods (Perrière and Thioulouse 2002; Nicolas et al. 2002; Lafay et al. 2000) or with numerical criteria based on ribosomal analysis (Carbone et al. 2004).

\section{The Case of $\mathrm{H}$. Pylori}

H. pylori is a microaerophilic, Gram-negative bacterium, whose infection is associated with type B gastritis and peptic ulcer disease and is a risk factor for gastric carcinomas in humans. H. pylori is a slow grower. It has been cultured on diverse agar-based media, resulting in 2 to 4 days of growth at $37^{\circ} \mathrm{C}$. Its genome is known to display no sharp dominant codon bias but, rather, a homogeneous codon composition (Lafay et al. 2000), with $\mu=0.55, \sigma=0.12$, and $\mu_{R}=0.59$. Despite this fact, we observe that the pathway for glycolysis (4) has a high RPI in H. pylori, and this indicates that some translational bias in this organism is nevertheless present. In this respect, note that glucose appears to be the only carbohydrate utilized by this bacterium (Tomb et al. 1997) and that rapid growth with a doubling time of about $50 \mathrm{~min}$ has been reported (Andersen et al. 1997). Our analysis also indicates the thioredoxin pathway (40) to have a rather high RPI value (see Fig. 1), and this is experimentally confirmed by the finding that $H . p y$ lori has a thioredoxin-dependent peroxiredoxin system playing a critical role in the defense against oxygen toxicity that is essential for survival and growth, even in microaerophilic environments (Baker et al. 2001). Another highly RPI ranked pathway is riboflavin biosynthesis (48), which turns out to play a crucial role in ferric iron reduction and iron acquisition by H. pylori (Worst et al. 1998). In fact, as other pathogenic bacteria, H. pylori encounters an ironlimiting environment when it attempts to colonize or invade a mammalian host.

To summarize, we proposed that the lifestyle of an organism can be deduced for translationally biased organisms by analyzing the set of most biased genes in the genome, and we observed that weakly translationally biased genomes also carry information on lifestyle. The case of $H$. pylori suggests that this rule might be extended to a much broader class of organisms. In support of this hypothesis, we found that the most biased genes of the cyanobacterium Thermosynechococcus elongatus have photosynthetic functions. Over the first 24 top CAI ranked genes there are 6 photosystem I and II proteins, 1 phyco- bilisome small core linker polypeptide, 2 phycocyanin subunits, 2 allophycocyanin subunits, 2 cytochrome b6 proteins, and 2 ribosomal proteins. There is no evidence that Thermosynechococcus elongatus is translationally biased, neither experimental nor computational (Carbone et al. 2004) (in particular, no compositional bias has been detected), but its most biased genes highly reflect the lifestyle of this organism. Again, Chlorobium tepidum, a Gram-negative bacterium of the green sulfur phylum, is an obligate anaerobic photolithoautotroph and lives in high-sulfide hot springs where anoxic layers containing reduced sulfur compounds are exposed to light. It is a thermophile, growing optimally at $48^{\circ} \mathrm{C}$. There is no evidence that $C$. tepidum is translationally biased, neither experimental nor computational (Carbone et al. 2004), but we note that its first 20 top CAI ranked genes contain 2 hydrogenase/sulfur reductases, 3 sulfite reductase subunits, 2 iron-sulfur cluster binding proteins, 1 ferrodoxin protein, and 1 ribosomal protein. These are proteins that characterize well the living conditions of these bacteria. Note that the strong GC3 bias of this genome does not prevent the genomic coding from revealing the crucial role of certain enzymes.

These observations lead us to conclude that there is still much to understand about the role of codon bias in genomes and that refined quantitative measures of codon bias which are able to differentiate bias strengths are needed to investigate in silico a new range of biological possibilities for larger classes of organisms.

\section{Dominant Codon Bias and tRNA Charging Pathways}

Charging of tRNA pathways has been described for $E$. coli, A. tumefaciens, and $V$. cholerae among the organisms listed in Table 1. For these organisms most amino acids have a tRNA charging pathway $\mathrm{P}$ with $\mathrm{PI}(\mathrm{P}) \geq \mu+\sigma$. Namely, E. coli, A. tumefaciens, and $V$. cholerae have 20,16 , and 18 pathways $\mathrm{P}$ with $\mathrm{PI}(\mathrm{P})$, $\geq \mu+\sigma$ respectively. This finding leads to the hypothesis that all translationally biased genomes present high bias on tRNA charging pathways. We also verified that in $H$. pylori, 19 tRNA charging pathways $\mathrm{P}$ display $\mathrm{RPI}(\mathrm{P}) \geq \mu$ and 12 of them have $\operatorname{RPI}(\mathrm{P}) \geq \mu_{R}$. This last observation suggests a need for efficiency of this process which is independent of the strength of translational bias. Note also that, for $E$. coli, A. tumefaciens, and $V$. cholerae, we separated two sets of genes, one containing the $2 \%$ of most biased genes and the other containing all other genes. We then applied linear discriminant analysis and observed that the amino acid separation coefficients do not display a significant correlation between the organism-preferred codons and the specific codons of the tRNA charging complexes displaying a high RPI. The availability of 
further metabolic information on genomes will allow us to verify the hypothesis for a class of organisms displaying strong and weak forms of translational bias.

\section{$P I, P I_{T}$, and "Logically" Differentiated Regulation for Unicellular Organisms}

Constitutive high expression of rarely used but genomically favored (i.e., with high PI) pathways is expected to be avoided by specific patterns of regulation, and the existence of such patterns can be detected by combining sequence analysis and transcriptomic data: the four logical combinations coming from "high" and "low" $\mathrm{PI}_{\mathrm{T}}$ and PI values lead us to envisage at least four different kinds of enzyme regulation. Given a set of experiments, it is reasonable to distinguish the regulation of enzymes in a pathway P which has "high" $\mathrm{PI}(\mathrm{P})$ and "low" $\mathrm{PI}_{\mathrm{T}}(\mathrm{P})$ for all (or most) time point experiments from the regulation of a pathway $\mathrm{P}^{\prime}$ where $\mathrm{PI}_{\mathrm{T}}\left(\mathrm{P}^{\prime}\right)$ and $\mathrm{PI}\left(\mathrm{P}^{\prime}\right)$ are always "high." Enzymes participating in $\mathrm{P}^{\prime}$ are likely to be constitutively regulated, while it is plausible that enzymes participating in $\mathrm{P}$ are regulated under specific biological conditions (as in the case of seripauperine or Hem 13 proteins discussed above). The number of "logically" foreseeable regulation modes becomes larger if we consider the frequency at which high and low $\mathrm{PI}_{\mathrm{T}}(\mathrm{P})$ values, for a pathway $\mathrm{P}$, occur in experiments. This logic-based diversity characterizing metabolic pathways challenges the understanding of the underlying regulatory mechanisms. The interplaying role of statistical analysis and transcriptomic data turns out to be essential here.

\section{Caveats to the Analysis Based on Transcriptomic Data}

A major concern with our analysis based on microarray data is the noisiness of transcriptomic data and the limited correlation between mRNA and protein expression levels. However, the amount of noise is smaller and the correlation higher for highly expressed genes (Gygi et al. 1999), those of most interest in our analysis.

\begin{abstract}
Acknowledgments. Part of this work was done while the authors were visiting the Institut des Hautes Études Scientifiques. The authors are grateful to David Fell for his encouragement of this work and to Jean-Michel Camadro, Nadya Morozova, Eric Stewart, and Catherine Vaquero for stimulating conversations and insights. A.C. is supported by a grant from the Fondation pour la Recherche Médicale.
\end{abstract}

\section{References}

Akashi H (2001) Gene expression and molecular evolution. Curr Opin Genet Dev 11:660-666
Akashi H (2003) Translational selection and yeast proteome evolution. Genetics 164:291-1303

Akashi H, Gojobori T (2002) Metabolic efficiency and amino acid composition in the proteomes of Escherichia coli and Bacillus subtilis. Proc Natl Acad Sci USA 99:3695-3700

Amillet JM, Buisson N, Labbe Bois R (1995) Positive and negative elements involved in the differential regulation by heme and oxygen of the HEM13 gene (coproporphyrinogen oxidase) in Saccharomyces cerevisiae. Curr Genet 28:503-511

Andersen AP, Elliott DA, Lawson M, Barland P, Hatcher VB, Puszkin EG (1997) Growth and morphological transformations of Helicobacter pylori in broth media. J Clin Microbiol 35:29182922

Baker LMS, Raudonikiene A, Hoffman PS, Poole LB (2001) Essential thioredoxin-dependent peroxiredoxin system from Helicobacter pylori: Genetic and kinetic characterization. J Bacteriol 183:1961-1973

Carbone A, Zinovyev A, Képès F (2003) Codon Adaptation Index as a measure of dominating codon bias. Bioinformatics 19:2005-2015

Carbone A, Képès F, Zinovyev A (2004) Codon bias signatures, organisation of microorganisms in codon space and lifestyle. Mol Biol Evol 22:547-561

Cohen NG, et al. (2003) An integrated analysis of the genome of the hyperthermophilic archaeon Pyrococcus abyssi. Mol Microbiol 47:1495-1512

deRisi JL, Iyer VR, Brown PO (1997) Exploring the metabolic and genetic control of gene expression on a genomic scale. Science 278:680-686

Gombert AK, Moreira dos Santos M, Christensen B, Nielsen J (2001) Network identification and flux quantification in the central metabolism of Saccharomyces cerevisiae under different conditions of glucose repression. J Bacteriol 183:1441-1451

Gouy M, Gautier Ch (1982) Codon usage in bacteria: correlation with gene expressivity. Nucleic Acids Res 10:7055-7070

Gygi SP, Rochon Y, Franza BR, Aebersold R (1999) Correlation between protein and mRNA abundance in yeast. Mol Cell Biol 19:1720-1730

Helling BR (1994) Why does Escherichia coli have two primary pathways for synthesis of glutamate? J Bacteriol 176:4664-4668

Holstege FCP, Jennings EG, Wyrick JJ, Lee TI, Hengartner CJ, Green MR, Golub TR, Lander ES, Young RA (1998) Dissecting the regulatory circuitry of a eukaryotic genome. Cell 95:717-728

Karp PD, Riley M, Paulsen IT, Paley S, Pellegrini-Toole A (2000) The EcoCyc and MetaCyc Databases. Nucleic Acids Res 28:56-59

Karp PD, Riley M, Saier M, Paulsen IT, Collado-Vides J, Paley S, Pellegrini-Toole A, Bonavides C, Gama-Castro S (2002) The EcoCyc Database. Nucleic Acids Res 30:56-58

James TC, Campbell S, Donnelly D, Bond U (2003) Transcription profile of brewery yeast under fermentation conditions. J Appl Microbiol 94:432-448

Jeppsson M, Johansson B, Hahn-Hägerdal B, Gorwa-Grauslund MF (2002) Reduced oxidative pentose phosphate pathway flux in recombinant xylose-utilizing Saccharomyces cerevisiae strains improves the ethanol yield from xylose. Appl Environ Microbiol 68:1604-1609

Johnston M, Carlson M (1992) Regulation of carbon and phosphate utilization. In: Jones EW, Pringle JR, Broach JR (eds). The molecular biology of the yeast Saccharomyces: Gene expression. Cold Spring Harbor Laboratory Press, Cold Spring Harbor, NY, 1992, pp 193-281

Lafay B, Atherton JC, Sharp PM (2000) Absence of translationally selected synonymous codon usage bias in Helicobacter pylori. Microbiology 146:851-860

Médigue C, Rouxel T, Vigier P, Hénaut A, Danchin A (1991) Evidence for horizontal gene transfer in Escherichia coli speciation. J Mol Biol 222:851-856 
Middelhoven WJ, Kurtzman CP, Vaughan-Martini A (2000) Saccharomyces bulderi sp. nov., a yeast that ferments gluconolactone. Antonie van Leeuwenhoek 77:223-228

Mrázek J, Bhaya D, Grossman AR, Karlin S (2001) Highly expressed and alien genes of the Synechocystis genome. Nucleic Acids Res 29:1590-1601

Newman EB, Walker C (1982) L-Serine degradation in Escherichia coli K-12: A combination of L-serine, glycine, and leucine used as a source of carbon. J Bacteriol 151:777-782

Nicolas P, Bize L, Muri F, Hoebeke M, Rodolphe F, Dusko Ehrlich S, Prum B, Bessières Ph (2002) Mining Bacillus subtilis chromosome heterogeneities using hidden Markov models. Nucleic Acids Res 30:1418-1426

Norman E, De Smet KA, Stoker NG, Ratledge C, Wheeler PR, Dale JW (1994) Lipid synthesis in mycobacteria: characterization of the biotin carboxyl carrier protein genes from Mycobacterium leprae and M. tuberculosis. J Bacteriol 176:2525-2531

Parish T, Stoker NG (2002) The common aromatic amino acid biosynthesis pathway is essential in Mycobacterium tuberculosis. Microbiology 148:3069-3077

Perrière G, Thioulouse J (2002) Use and misuse of correspondence analysis in codon usage studies. Nucleic Acids Res 30:4548-4555

Pizer IL, Potochny LM (1964) Nutritional and regulatory aspects of serine metabolism in Escherichia coli. J Bacteriol 88:611-619

Primm TP, Andersen SJ, Mizrahi V, Avarbock D, Rubin H, Barry CE 3rd (2000) The stringent response of Mycobacterium tuberculosis is required for long-term survival. J Bacteriol 182:4889-4898

Ratledge C (1991) Yeast physiology-A micro-synopsis. Bioprocess Eng 6:195-203

Reitzer LJ (1986) Ammonia assimilation and the biosynthesis of glutamine, glutamate, aspartate, asparagine, L-alanine, and Dalanine. In: Neidhardtf C, Ingraham JL, Low KB, Magasanik B, Schaechter M, Umbarger HE (eds). Escherichia coli and Salmonella typhimurium: Cellular and molecular biology. American Society for Microbiology, Washington, DC, pp 302-320

Sassetti CM, Boyd DH, Rubin EJ (2003) Genes required for mycobacterial growth defined by high density mutagenesis. Mol Microbiol 48:77-84

Schut GJ, Zhou J, Adams MW (2001) DNA microarray analysis of the hyperthermophilic archaeon Pyrococcus furiosus: Evidence for a new type of sulfur-reducing enzyme complex. J Bacteriol 183:7027-7036

Sharp PM, Li W-H (1987) The codon adaptation index-A measure of directional synonymous codon usage bias, and its potential applications. Nucleic Acids Res 15:1281-1295
Sharp PM, Tuohy TMF, Mosurski KR (1986) Codon usage in yeast: cluster analysis clearly differentiate highly and lowly expressed genes. Nucleic Acids Res 14:8207-8211

Sharp PM, Cowe E, Higgins DG, Shields DC, Wolfe KH, Wright F (1988) Codon usage patterns in Escherichia coli, Bacillus subtilis, Saccharomyces pombe, Drosophila melanogaster and Homo sapiens; A review of the considerable within species diversity. Nucleic Acids Res 16:8207-8211

Shields DC, Sharp PM (1987) Synonymous codon usage in Bacillus subtilis reflects both traditional selection and mutational biases. Nucleic Acids Res 15:8023-8040

Silva PJ, van den Ban EC, Wassink H, de Haaker HCB, Robb FT, Hagen WR (2000) Enzymes of hydrogen metabolism in Pyrococcus furiosus. Eur J Biochem 267:6541-6551

Sinha A, Maitra PK (1992) Induction of specific enzymes of the oxidative pentose phosphate pathway by glucono-delta-lactone in Saccharomyces cerevisiae. J Gen Microbiol 138:18651873

Stenico M, Loyd AT, Sharp PM (1994) Codon usage in Caenorhabditis elegans: delineation of translational selection and mutational biases. Nucleic Acids Res 22:2437-2446

Tomb JF, et al. (1997) The complete genome sequence of the gastric pathogen Helicobacter pylori. Nature 388:539-547

van Dijken JP, van Tuijl A, Luttik MAH, Middelhoven WJ, Pronk JT (2002) Novel pathway for alcoholic fermentation of $\delta$-gluconolactone in the yeast Saccharomyces bulderi. J Bacteriol 184:672-678

Viswanathan M, Muthukumar G, Cong YS, Lenard J (1994) Seripauperins of Saccharomyces cerevisiae: A new multigene family encoding serine-poor relatives of serine-rich proteins. Gene 148:149-153

Wagner A (2000) Inferring lifestyle from gene expression patterns. Mol Biol Evol 17:1985-1987

Ward DE, Kengen SW, Van der Oost J, De Vos WM (2000) Purification and characterization of the alanine aminotransferase from the hyperthermophilic Archaeon Pyrococcus furiosus and its role in alanine production. $\mathbf{J}$ Bacteriol 182:2559-2566

Wood WD, et al. (2001) The genome of the natural genetic engineer Agrobacterium tumefaciens C58. Science 294:2317-2323

Worst DJ, Gerrits MM, Vandenbroucke-Grauls CMJE, Kusters JG (1998) Helicobacter pylori ribBA-mediated riboflavin production is involved in iron acquisition. J Bacteriol 180:14731479

Zitomer RS, Lowry CV (1992) Regulation of gene expression by oxygen in Saccharomyces cerevisiae. Microbiol Rev 56:1-11 
org path meancai rpi
yeast sorbitol degradation Yeast sorbitol degradation
vcho sorbitol degradation ecoli sorbitol degradation bsub sorbitol degradation hin sorbitol degradation yeast mannitol degradatio veho mannitol degradation ecolt mannitol degradatio bsub mannitol degradation

hin mast glutamine biosynthesi

0.69044563032330708 0.63109074155285627 0.4894085406638351 0.56443870613843428 0.69044563032330708 0.5054354990004543999 0.564173005061635027 0.54953273363146293 $\begin{array}{ll}0 & 0\end{array}$ ecoli glutamine biosynthesis 0.61006042249898129 bsub glutamine biosynthesis 0.57962263262381308 $\begin{array}{lll}\text { hin } & \text { glutamine biosynthesis } & 0.4817244296967782 \\ \text { aqro glutamine biosynthesis } & 0.57474706764068884\end{array}$ mtbrv glutamine biosynthesis 0.6011138177829275 yeast glycolysis 0.7321643393112032 vcho glycolysis 0.5252588756316553 ecoli glycolysis 0.5803379983785724 $\begin{array}{lll}\text { bsub glycolysis } & 0.5522090743825977 \\ \text { hin } & \text { glycolysis } & 0.5822708526499705\end{array}$ $\begin{array}{lll}\text { hagro glycolysis } & 0.5399838582406387\end{array}$ metbrv glycolysis $\quad 0.5739618260192808$ hpy glycolysis 0.60410001935447
vcho pyruvate oxidation pathway 3.4915552932180631
2.2225280640101492 2.222528064010149 2.4796095046642725
2.4432130217789139 2.1553934466861917 0.47227132192543075
0.33152671892207203

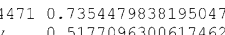
ecoli pyruvate oxidation pathway 0.4744920557295616 bsub pyruvate oxidation pathway $\quad 0.54632510027935666$ hin pyruvate oxidation pathway 0.5369083224580935 yeast pyruvate dehydrogenase 0.31644122380609985 $\begin{array}{lll}\text { vcho pyruvate dehydrogenase } & 0.58686666039492019 \\ \text { ecoli pyruvate dehydrogenase } & 0.62632640151318886\end{array}$ $\begin{array}{ll}\text { ecoli pyruvate dehydrogenase } & 0.62632640151318886 \\ \text { bsub pyruvate dehydrogenase } & 0.56832726449748128\end{array}$ $\begin{array}{ll}\text { hin pyruvate dehydrogenase } & 0.55237535592802833 \\ \text { agro pyruvate dehydrogenase } & 0.651486147383322 \\ \text { mtbrv pyruvate dehydrogenase } & 0.52902570372410684\end{array}$ yeast glycine cleavage 0.4507795480917719 vcho glycine cleavage 0.40054405108973751
ecoli glycine cleavage 0.5223900335743511 $\begin{array}{ll}\text { ecoli glycine cleavage } & 0.5223900335743511 \\ \text { bsub glycine cleavage } & 0.55329059715944495\end{array}$ $\begin{array}{lll}\text { bsub glycine cleavage } & 0.55329059715944495 \\ \text { hin } & \text { glycine cleavage } & 0.53517520542301689\end{array}$ $\begin{array}{lll}\text { hin glycine cleavage } & 0.53517520542301689 \\ \text { agro glycine cleavage } & 0.59809850530902309\end{array}$ mtbrv glycine cleavage 0.59239689026003384 yeast gluconeogenesis 0.60472923305046022 $\begin{array}{ll}\text { vcho gluconeogenesis } & 0.48868662556786563\end{array}$ $\begin{array}{ll}\text { ecoli gluconeogenesis } & 0.5399459603886162 \\ \text { bsub gluconeogenesis } & 0.49517271643050836\end{array}$ $\begin{array}{lll}\text { bsub gluconeogenesis } & 0.49517271643050836 \\ \text { hin } & \text { gluconeogenesis } & 0.56938321443868811\end{array}$ $\begin{array}{lll}\text { hin gluconeogenesis } & 0.56938321443868811 \\ \text { agro gluconeogenesis } & 0.52620417529151542\end{array}$ $\begin{array}{ll}\text { agro gluconeogenesis } & 0.52620417529151542 \\ \text { mtbry gluconeogenesis } & 0.56609656310424228\end{array}$ hpy gluconeogenesis 0.5999407537795638 yeast removal of superon yeast removal of superd
0.051247363687748376 de radicals vcho removal of superoxide

1.1
0.6
1.7
2.4
1.4
1.
0.7
2.4
1.
1.
1.
1.
0.
0.
0.

$$
\begin{array}{ll}
833222.2456752492993837 \\
2410684 \quad-0.59778239860349425 \\
1 & 1.1549774312703336 \\
1 & 0.6241603225913378 \\
& 1.7392258095646216 \\
5 & 2.4613475212813234 \\
9 & 1.478038106566544 \\
9 & 1.3965639453454115 \\
4 & 0.71277619943056814 \\
2 & 2.4333529403709049 \\
3 & 1.753811494461523 \\
& 1.963532594503254 \\
6 & 1.4868523911534755 \\
1 & 1.970036389767204 \\
2 & 0.25311040560852305 \\
8 & 0.1688678189248137 \\
0.30551868706742347238268 \\
0
\end{array}
$$

\begin{tabular}{|c|c|c|c|c|c|}
\hline ecoli & removal of superoxide & radicals & \multicolumn{3}{|c|}{0.57548685862371951} \\
\hline bsub & $\begin{array}{l}\text { removal of superoxide } \\
0.83282601148576407\end{array}$ & radicals & \multicolumn{3}{|c|}{0.45616726021840859} \\
\hline hin & $\begin{array}{l}\text { removal of superoxide } \\
2.9072684503447972\end{array}$ & radicals & \multicolumn{3}{|c|}{0.63454775641789052} \\
\hline agro & $\begin{array}{l}\text { removal of superoxide } \\
1.5290521235488643\end{array}$ & radicals & \multicolumn{3}{|c|}{0.60642866344502322} \\
\hline $\begin{array}{l}\text { mtbrv } \\
0.1525\end{array}$ & $\begin{array}{l}\text { removal of superoxide } \\
54242710250413\end{array}$ & radicals & \multicolumn{3}{|c|}{0.55055498545905479} \\
\hline hpy & removal of superoxide & radicals & \multicolumn{3}{|c|}{0.61786088698048958} \\
\hline yeast & $\begin{array}{l}\text { non-oxidative branch } \\
0.94631509678196424\end{array}$ & of the pentose & \multicolumn{3}{|c|}{ phosphate pathway 0.42565117193895424} \\
\hline vcho & $\begin{array}{l}\text { non-oxidative branch } \\
2.0259660004711724\end{array}$ & of the pentose & \multicolumn{3}{|c|}{ phosphate pathway 0.50992185234807696} \\
\hline ecoli & $\begin{array}{l}\text { non-oxidative branch } \\
1.097064945011438\end{array}$ & of the pentose & \multicolumn{3}{|c|}{ phosphate pathway 0.47212972203039177} \\
\hline bsub & $\begin{array}{l}\text { non-oxidative branch } \\
1.3340657575049684\end{array}$ & of the pentose & \multicolumn{3}{|c|}{ phosphate pathway 0.48606067976984829} \\
\hline hin & $\begin{array}{l}\text { non-oxidative branch } \\
0.59348217568410244\end{array}$ & of the pentose & \multicolumn{3}{|c|}{ phosphate pathway 0.47367316711595275} \\
\hline agro & $\begin{array}{l}\text { non-oxidative branch } \\
1.7049715775347944\end{array}$ & of the pentose & \multicolumn{3}{|c|}{ phosphate pathway 0.61748955122} \\
\hline mtbrv & $\begin{array}{l}\text { non-oxidative branch o } \\
1.2915831805275817\end{array}$ & of the pentose & \multicolumn{3}{|c|}{ phosphate pathway 0.62038471562123321} \\
\hline hpy & $\begin{array}{l}\text { non-oxidative branch o } \\
0.1811769824974791\end{array}$ & the pentose & \multicolumn{3}{|c|}{ phosphate pathway 0.5908850414811937} \\
\hline vcho & $\begin{array}{l}\text { salvage pathways of gu } \\
0.3438703161385897\end{array}$ & $\begin{array}{l}\text { yanine, xanthin } \\
-0.102180511\end{array}$ & \multicolumn{3}{|c|}{$\begin{array}{l}\text { ine, and their nucleosides } \\
1190334468\end{array}$} \\
\hline ecoli & $\begin{array}{l}\text { salvage pathways of gu } \\
0.47406932053935164\end{array}$ & $\begin{array}{r}\text { yanine, xanthin } \\
1.1218466110\end{array}$ & \multicolumn{3}{|c|}{$\begin{array}{l}\text { ne, and their nucleosides } \\
0444936\end{array}$} \\
\hline hin & $\begin{array}{l}\text { salvage pathways of gr } \\
0.47379167726318799\end{array}$ & $\begin{array}{r}\text { guanine, xanthin } \\
0.5951866533\end{array}$ & \multicolumn{3}{|c|}{$\begin{array}{l}\text { ine, and their nucleosides } \\
339727495\end{array}$} \\
\hline agro & $\begin{array}{l}\text { Salvage pathways of gu } \\
0.53711742145249397\end{array}$ & $\begin{array}{r}\text { yanine, xanthin } \\
0.4266816726\end{array}$ & \multicolumn{3}{|c|}{$\begin{array}{l}\text { ine, and their nucleosides } \\
62988771\end{array}$} \\
\hline hpy & $\begin{array}{l}\text { salvage pathways of gh } \\
0.65263465016903366\end{array}$ & $\begin{array}{l}\text { yuanine, xanthin } \\
2.7711183177\end{array}$ & \multicolumn{3}{|l|}{$\begin{array}{l}\text { Ine, and } t \\
7654353\end{array}$} \\
\hline yeast & $\begin{array}{l}\text { methyl-donor molecule } \\
2.1533940340236168\end{array}$ & biosynthesis & \multicolumn{3}{|c|}{0.5710148939327877} \\
\hline vcho & $\begin{array}{l}\text { methyl-donor molecule } \\
0.47935649818561815\end{array}$ & thesis & \multicolumn{3}{|c|}{0.38924553505629483} \\
\hline ecoli & $\begin{array}{l}\text { methyl-donor molecule } \\
1.0663995659348344\end{array}$ & thesis & \multicolumn{3}{|c|}{0.46972962013835867} \\
\hline bsub & $\begin{array}{l}\text { methyl-donor molecule } \\
1.6445774315756501\end{array}$ & biosynthesis & \multicolumn{3}{|c|}{0.5045792745587363} \\
\hline hin & $\begin{array}{l}\text { methyl-donor molecule } \\
0.84592966510031975\end{array}$ & biosynthesis & \multicolumn{3}{|c|}{0.49122551696991446} \\
\hline agro & $\begin{array}{l}\text { methyl-donor molecule } \\
0.34223951684377663\end{array}$ & & \multicolumn{3}{|c|}{0.53180814389594944} \\
\hline mtbrv & $\begin{array}{l}\text { methyl-donor molecule } \\
1.9270883300085879\end{array}$ & & \multicolumn{3}{|c|}{0.65111414308271043} \\
\hline hpy & $\begin{array}{l}\text { methyl-donor molecule } \\
499709962401\end{array}$ & biosynthesis & \multicolumn{3}{|c|}{61480232451} \\
\hline & tRNA charging pathw & & 68482462 & 1.04490 & 9095355 \\
\hline & tRNA charging $\mathrm{p}$ & & & & \\
\hline & tRNA $\mathrm{c}$ & & & & \\
\hline & & & & & \\
\hline & tRNA 0 & & & & \\
\hline & anaerobic respiration & & 87224902 & & \\
\hline
\end{tabular}

0.40168824448967289 mtbrv oxidative branch of the pentose phosphate pathway 0.58485255057498142 hpy $\begin{aligned} & 0.55675419982464891 \\ & \text { oxidative branch of the pentose phosphate pathway } \\ & -1.1625535833703473\end{aligned}$ 0.55884769936292389 yeast asparagine biosynthesis and degradation 0.37372077889859756 vcho asparagine biosynthesis and degradation 0.37363317629875137 ecoli asparagine biosynthesis and degradation 0.42815851467034749 0.53525807063349662
0.38453528853519647 bsub asparagine biosynthesis and degradation 0.38453528853519647
0.36826746768493374 hin asparagine biosynthesis and degradation 0.49084934257679275 mtbrv asparagine biosynthesis and degradation 0.61440138062089444 vcho NAD phosphorylation and dephosphorylation 0.33505385382186631 VCho NAD phosphorylation and dephosphorylation 0.33505385382186631
0.21517388708745364 ecoli NAD phosphorylation and dephosphorylation 0.3644797756951258 agro NAD phosphorylation and dephosphorylation 0.61323163400441949 mtbrv NAD phosphorylation and dephosphorylation 0.59151470966061781 0.69453211354891808
yeast fatty acid biosynthesis, initial steps 0.35722304482209549 vcho fatty acid biosynthesis, initial steps 0.39270796668882413 ecoli fatty acid biosynthesis, initial steps 0.45746884653728531
0.523716480415427 bsub fatty acid biosynthesis, initial steps 0.44252997714690773 $\begin{array}{ll}\text { bsub fatty acid biosynthesis, initial steps } & 0.44252907714690773 \\ & 0.60416203106061805 \\ \text { hin fatty acid biosynthesis, initial steps } & 0.48600588747184137\end{array}$

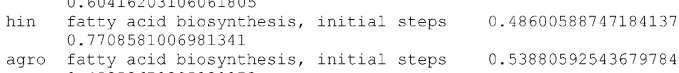
$\begin{array}{ll}\text { agro fatty acid biosynthesis, initial steps } & 0.53880592543679784 \\ 0.45353672305238873 & \\ \text { mtbry fats } & \end{array}$ $\begin{array}{ll}\text { mtbrv fatty acid biosynthesis, initial steps } & 0.57036061828943907 \\ & 0.25705133015883436\end{array}$ hpy fatty acid biosynthesis, initial steps 0.5810854796613516
0.22984246789331716
vcho salvage pathways of adenine, hypoxanthine, and their nucleosides
$0.34946463907479403 \quad-0.030482657625380005$ ecoli salvage pathways of adenine, hypoxanthine, and their nucleosides
0.4939334642652303
0.3756432876937434 bsub salvage pathways of adenine, hypoxanthine, and their nucleosides hin $0.4499688050688091 \quad 0.72889303454579257$. hin salvage pathways of adenine, hypoxanthine, and their nucleosides
0.52627571969080433
1.35004083847811 agro salvage pathways of adenine, hypoxanthine, and their nucleosides $\begin{aligned} & \text { mtbrv salvage pathways of adenine, hypoxanthine, and their nucleosides } \\ & 0.51763163548488589-0.8334193641356471\end{aligned}$ hpy salvage pathways of adenine, hypoxanthine, and their nucleosides yeast UDP-N-acetylglucosamine biosynthesis 0.29647433209423935

\begin{tabular}{|c|c|}
\hline & $\begin{array}{l}562577754468902 \\
0108718352091898 \\
1042201126136 \\
52060161848 \\
14625038220868 \\
824512605918207 \\
1132245368888907 \\
580054143406779 \\
66416008150956 \\
56676712255128 \\
5780413804323 \\
500375714038699\end{array}$ \\
\hline \multicolumn{2}{|c|}{0.49196676819673507} \\
\hline \multicolumn{2}{|c|}{0.4430307164498391} \\
\hline \multicolumn{2}{|c|}{0.44733175637109479} \\
\hline \multicolumn{2}{|c|}{0.56605481414307302} \\
\hline \multicolumn{2}{|c|}{0.54577124345870087} \\
\hline \multicolumn{2}{|c|}{0.61164842324424928} \\
\hline \multirow{2}{*}{\multicolumn{2}{|c|}{$\begin{array}{l}0.5556089740136495 \\
0.010248536655117212 \\
1.6881129890439637 \\
2.4613475212813234 \\
0.32501307350633118 \\
1.208705769368672 \\
0.073863762422954116 \\
-1.357205277098755 \\
0.1092781396140616 \\
0.80375957352769078 \\
0.134068379498552 \\
1.3475259512778464 \\
1.4618770205482583 \\
0.055278448737250289 \\
9452805087337 \\
3763858650422 \\
6432417319472 \\
8042853925686 \\
0394736547591 \\
080886157112 \\
0892100252043 \\
163289098493 \\
0 \\
0.42205103830715995\end{array}$}} \\
\hline & \\
\hline & 0.47308113642192584 \\
\hline & 0.4637124755694263 \\
\hline & 0.43969025003959061 \\
\hline & \\
\hline & 0.5191503320126842 \\
\hline
\end{tabular}
0.12635035521167878
vcho UDP-N-acetylglucosamine biosynthesis $\quad 0.34095044677184716$ ecoli UDP-N-acetylglucosamine biosynthesis $\quad 0.41386178847746868$
0.34095044677184716 bsub oxidative branch of the pentose phosphate pathway

hin oxidative branch of the pentose phosphate pathway agro oxidative branch of the pentose phosphate pathway
0.42205103830715995 0.47308113642192584 0.4637124755694263 0.43969025003959061 0.5008470497662727 0.51915033201268423 


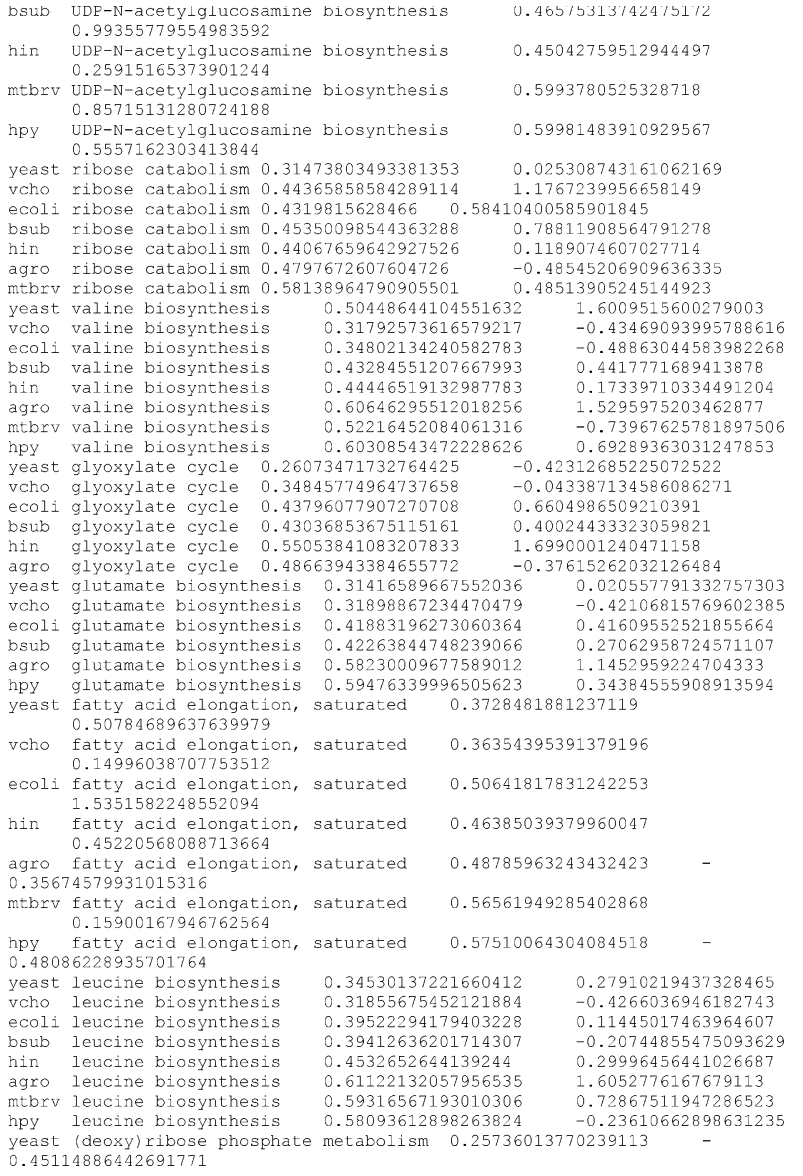

vcho (deoxy) ribose phosphate metabolism $0.38588 / 9103900 / 5 b 3$ ecoli 0.43632457336040831 . $\begin{array}{ll}\text { ecoli (deoxy) ribose phosphate metabolism } & 0.40907739676052854 \\ 1.4413672696651023 & \end{array}$ bsub (deoxy) ribose phosphate metabolism 0.45602491057068417
0.83043915503568377 hin (deoxy) ribose phosphate metabolism 0.43853330131961965 (deoxy) agro (deoxy) ribose phosphate metabolism 0.49416840123583644
0.25640709366098596 mtbrv (deoxy)ribose phosphate metabolism 0.55372171926198122 0.08705225025768322 photatism 0.58976752768378149

hpy (deoxy) ribose phosphate metabolism 0.58976752768378149
0.13430550852131942
vcho de novo biosynthesis of pyrimidine ribonucleotides 0.38412029630634176
0.4136705116552738 ecoli de novo biosynthesis of pyrimidine ribonucleotides 0.44245027745143478 bsub de novo biosynthesis of pyrimidine ribonucleotides 0.40641392394406622 hin de novo biosynthesis of pyrimidine ribonucleotides $\quad 0.4143255384811122$ -0.26008785774876664
agro de $\begin{array}{lll}\text { agro de novo biosynthesis of pyrimidine ribonucleotides } & 0.54175725117254547 \\ 0.50047650062087579 & \text { a p primidine ribonucleotides } & 0.56422670843578981\end{array}$ mtbrv de novo biosynthesis of pyrimidine ribonucleotides 0.56422670843578981
0.13019796449384985 hpy de novo biosynthesis of pyrimidine ribonucleotides 0.59423268583878475 yeast glutathione-glutaredoxin redox reactions 0.25068135335338898

0.50660850596252516 dexin redox reactions 0.41788225625020065

vcho glutathione-glutaredoxin redox reactions 0.41788225625020065
0.84636989490461889 ecoli glutathione-glutaredoxin redox reactions 0.43941024755278241 hin glutathione-glutaredoxin redox reactions 0.48886427284993605 agro glutathione-glutaredoxin redox reactions 0.55644567196981165 mtbrv glutathione-glutaredoxin redox reactions 0.50429919768860465
1.1091431108697367

yeast TCA cycle, aerobic respiration $\quad 0.26888666616141943$
0.35543426981162735

vcho TCA cycle, aerobic respiration 0.35408222865337474

0.028697205334972491
ecoli TCA cycle, aerobic respiration $\quad 0.439847444445760643$

bsub TCA cycle, aerobic respiration $\quad 0.44774340239714649$

$\begin{array}{ll}\text { bsub TCA cycle, aerobic respiration } & 0.44774340239714649 \\ 0.69157845874039925 & 0.43144018915138438\end{array}$

hin TCA cycle, aerobic respiration
0.01393559723131743

$\begin{array}{ll}\text { agro TCA cycle, aerobic respiration } & 0.5323033533475386 \\ & 0.35011564380996146\end{array}$

yeast fatty acid elongation, unsaturated 0.35722866423138122
0.37814467052822753

vcho fatty acid elongation, unsaturated 0.35563069051462626
0.04854257253115437

ecoli fatty acid elongation, unsaturated 0.5041190556763031 .5057830273212069 hin fatty acid elongation, unsaturated 0.4585225944179524
0.37557835809555296

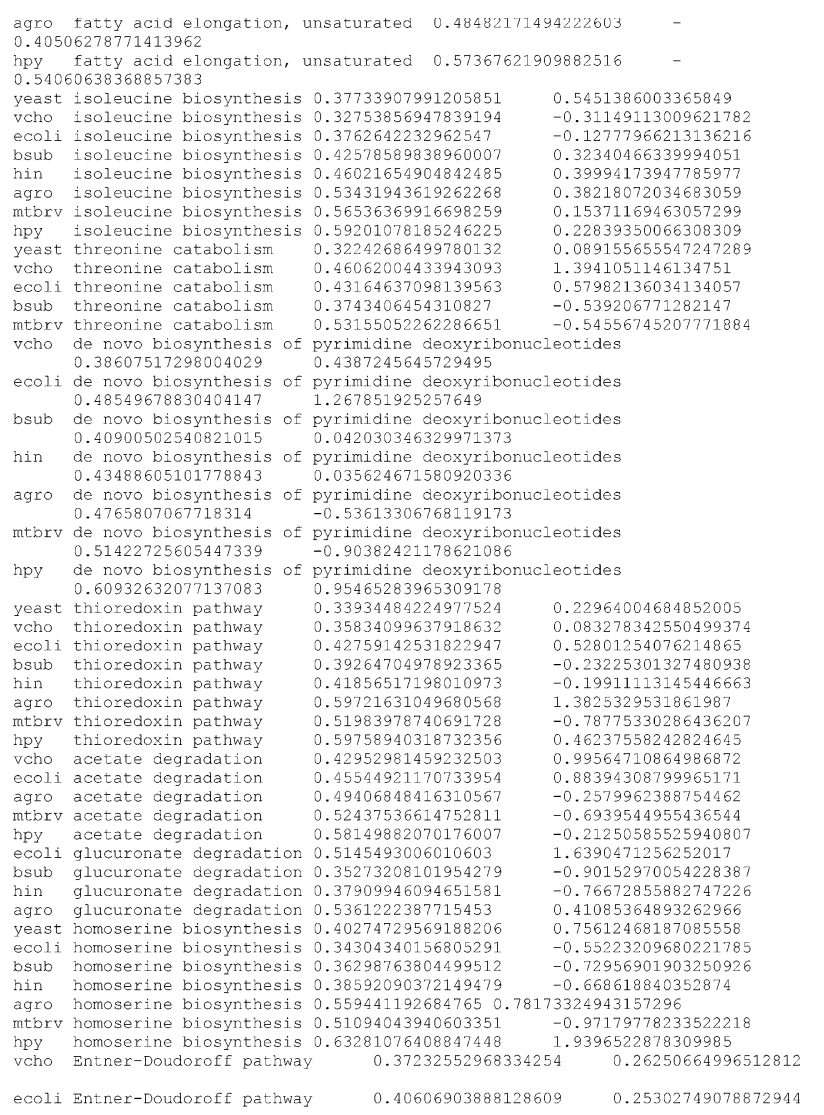

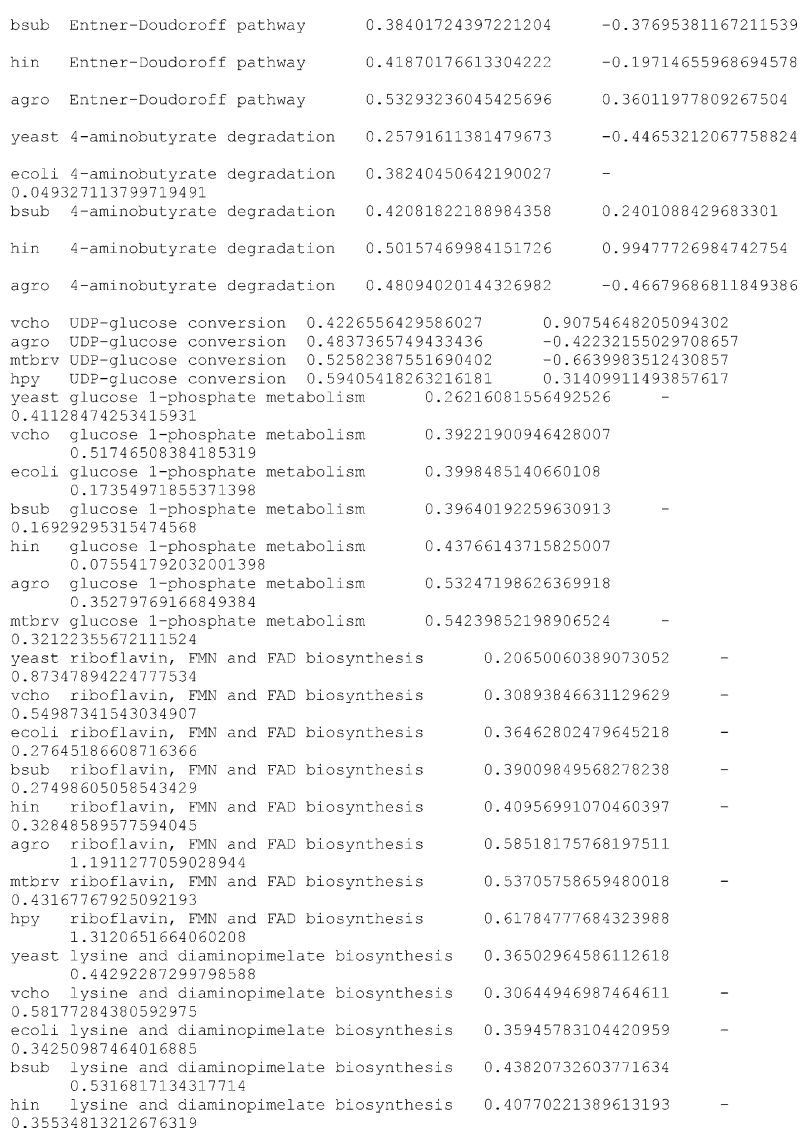




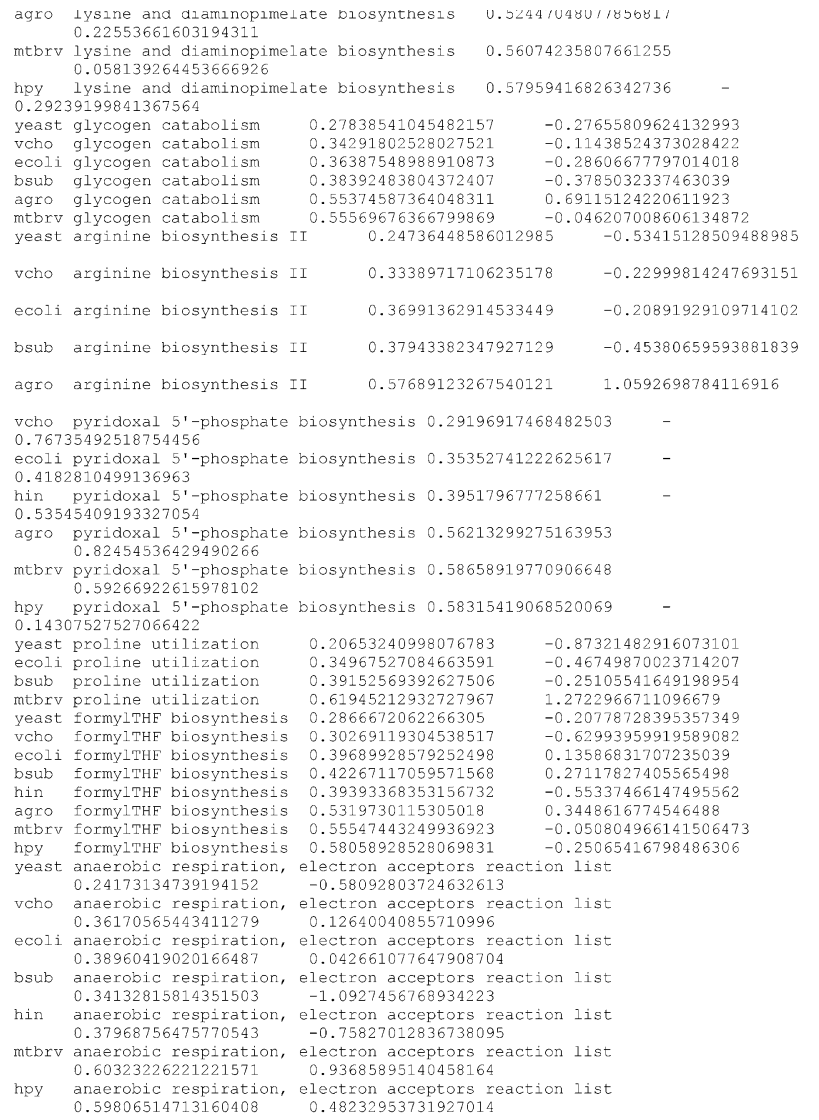

vcho glucosamine catabolism 0.33893638538442561 ecoli glucosamine catabolism $\quad 0.37203674309199031$ bsub glucosamine catabolism 0.35095983791400631 hin glucosamine catabolism 0.4211473696369890 agro glucosamine catabolism 0.50630869984864524

vcho sulfate assimilation pathway $0.28401562163428962 \quad-0.86928909919078123$ ecoli sulfate assimilation pathway $\quad 0.36703815262457912 \quad-0.24565838864442988$ bsub sulfate assimilation pathway $\quad 0.41405726094532913 \quad 0.12674401560748805$

agro sulfate assimilation pathway $0.53202361353549654 \quad 0.34566648419969981$

\begin{tabular}{|c|c|c|c|c|c|c|c|}
\hline east & glutamate degradation $V$ & & & & -0.7768 & & 9738 \\
\hline tho & glutamate degradation $\mathrm{V}$ & & & & & & \\
\hline oli & glutamate degradation $\mathrm{V}$ & & 796769 & & & & \\
\hline$u b$ & glutamate degradation $\mathrm{V}$ & 0.3497057374 & 4074443 & & -0.952274 & & \\
\hline & glutamate degradation $\mathrm{v}$ & 0.4768727099 & 9741917 & & & & \\
\hline & glutamate degradation $\mathrm{V}$ & $V 0.5597928792$ & 2141575 & & -1.122910 & 249299 & \\
\hline ast & $\begin{array}{l}\text { aspartate biosynthesis } \\
89896873059738\end{array}$ & and degradati & & 0.21 & 81313464936 & & \\
\hline & aspartate biosynthesis & and degradati & & 0.37 & 91365591819 & 1347 & \\
\hline coli & $\begin{array}{l}\text { aspartate biosynthesis } \\
0.89062541702785092\end{array}$ & and degradati & & 0.45 & 59722207496 & & \\
\hline ub & $\begin{array}{l}\text { aspartate biosynthesis } \\
27410269690527\end{array}$ & egradati & & 0.34 & 057374074 & 4432 & \\
\hline & $\begin{array}{l}\text { aspartate biosynthesis } \\
0.63949974988712155\end{array}$ & and degradati & & 0.47 & 68727099741 & 9179 & \\
\hline${ }^{\mathrm{PY}}$ & $\begin{array}{l}\text { aspartate biosynthesis } \\
9102492992356\end{array}$ & and degradati & & 0.55 & 97928792141 & & - \\
\hline east & $\begin{array}{l}\text { mannose and GDP-mannose } \\
0.91492655159249237\end{array}$ & e metabolism & 0.4218 & & 411450392 & & \\
\hline $\begin{array}{c}\text { cho } \\
808\end{array}$ & $\begin{array}{l}\text { mannose and GDP-mannose } \\
69114530630293\end{array}$ & metabolism & 0.28 & & 969269378 & & \\
\hline 0. & $\begin{array}{l}\text { and GDP-mannose } \\
551444\end{array}$ & & 0.3415 & 53162 & 5703675 & & \\
\hline & $\begin{array}{l}\text { mannose and GDP-mannose } \\
46108912072683\end{array}$ & e metabolism & 0.3778 & & 709957807 & & \\
\hline & $\begin{array}{l}\text { mannose and GDP-mannose } \\
8956049350323\end{array}$ & & & & 381623581 & & \\
\hline py & $\begin{array}{l}\text { mannose and GDP-mannose } \\
0.69609086097452455\end{array}$ & metabolism & 0.6031 & 16166 & 37641741 & & \\
\hline & & & & & & & \\
\hline & & & & & & & \\
\hline & folic a & 50 & 81 & & & & \\
\hline & folic acid b. & 0.4 & 5709 & & & 19 & \\
\hline & folic acid b. & 50.3 & 7931 & & -0 & 251 & \\
\hline & folic a & & 2042 & & & & \\
\hline & folic & 50.5 & & & & & \\
\hline & folic & 0.5 & 24 & & & & \\
\hline & & & & & & & \\
\hline & & & & & & & \\
\hline & & & & & & & \\
\hline & & & & & & & \\
\hline & chorismate biosynthesis & 0.3668077314 & 172036 & & -0 . & 830 & \\
\hline & chorismate hiosunthesis & 8. 0.5125251197 & 05795 & & 0.035549 & & 294 \\
\hline
\end{tabular}

$-0.16541466056864182$ 0.06331999737816990

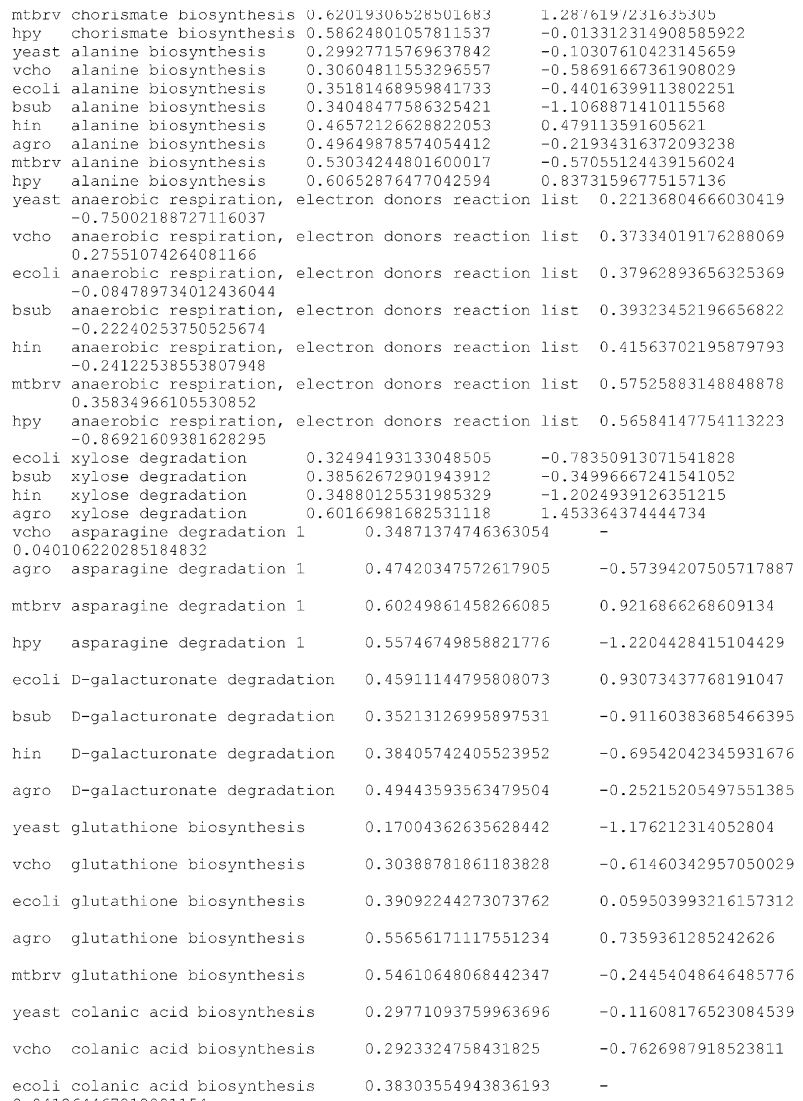

bsub colanic acid biosynthesis $0.38351465 / 2805 b 306 \quad-0.38538096485364 / 1$ b hin colanic acid biosynthesis $\quad 0.41641661058101881 \quad-0.23001291586338865$ agro colanic acid biosynthesis $\quad 0.46253165202043972 \quad-0.75957824592437384$ mtbrv colanic acid biosynthesis $\quad 0.5329993415841332 \quad-0.51560490520941327$ $\begin{array}{lll}\text { hpy colanic acid biosynthesis } & 0.6020244402716326 & 0.648392726701425\end{array}$ vcho salvage pathways of pyrimidine ribonucleotides 0.34661700227876174 ecoli salvage pathways of pyrimidine ribonucleotides 0.40106436227056269 bsub salvage pathways of pyrimidine ribonucleotides 0.40232052972307009 hin salvage pattways of pyrimidine ribonucleotides 0.39689561282685315
0.51077457540987059 agro salvage pathways of pyrimidine ribonucleotides 0.45873735390495901
0.81992519692742083 0.81992519692742083
mtbrv salvage pathways of pyrimidine ribonucleotides 0.48781050721095714 hpy salvage pathways of pyrimidine ribonucleotides 0.60547060273503217 yeast aerobic respiration, electron donors reaction list 0.23114367677927022 vcho aerobic respiration, electron donors reaction list 0.36225000825057624 vcho aerobic respiration, electron donors reaction list 0.36225000825057624
0.13337694549527446 $\begin{array}{cc}\text { ecoli aerobic respiration, electron donors reaction list } & 0.37536722855369004 \\ -0.13924029382444628 & \end{array}$ $\begin{array}{lll}\text { bsub aerobic respiration, electron donors reaction list } & 0.42128106379448221 \\ \text { hin } 0.24786957299551562 & \text { electron donors reaction list } & 0.39961231891481142\end{array}$ $\begin{array}{lll}\text { hin aerobic respiration, electron donors reaction list } & 0.39961231891481142 \\ \text { hpy aerobic respiration, electron donors reaction list } & 0.56739369536457873\end{array}$ $\begin{array}{llll}\text { hpy } & \text { aerobic respiration, electron donors reaction list } & 0.567393695364578 \\ \text { vcho galactose metabolism } & 0.29976725904648693 & -0.6674132660117057\end{array}$ \begin{tabular}{lll} 
vcho galactose metabolism & 0.29976725904648693 & -0.6674132660117057 \\
ecoli galactose metabolism & 0.4009123290735111 & 0.1871417625334563 \\
\hline
\end{tabular} $\begin{array}{lll}\text { ecoli galactose metabolism } & 0.4009123290735111 & 0.1871417625334563 \\ \text { bsub galactose metabolism } & 0.38348682455877969 & -0.3858476517232674 \\ \text { mtbry galactose metabolism } & 0.53783699298996002 & -0.41555903277488732\end{array}$ $\begin{array}{llll}\text { mtbrv galactose metabolism } & 0.53783699298996002 & -0.41555903277488732 \\ \text { yeast fatty acid oxidation pathway } & 0.22603547468656424 & -0.7112642523928453\end{array}$ vcho fatty acid oxidation pathway $0.37678707514989163 \quad 0.31968662337084419$ ecoli fatty acid oxidation pathway $0.41707732759132027 \quad 0.39367708045758248$ bsub fatty acid oxidation pathway $0.34264432278341767 \quad-1.0706768055590792$ hin fatty acid oxidation pathway $0.37108579152968973 \quad-0.8819855325159639$ agro fatty acid oxidation pathway $0.50394324233674681 \quad-0.10094175092144378$ mtbrv fatty acid oxidation pathway $0.58916014765519298 \quad 0.64583819365419726$ hpy fatty acid oxidation pathway $0.55599443860917175 \quad-1.2822268594197177$ yeast serine biosynthesis $\quad 0.27589284197132763 \quad-0.29725601780518157$ $\begin{array}{lll}\text { vcho serine biosynthesis } & 0.29530470769991307 & -0.7246061309576956 \\ \text { ecoli serine biosynthesis } & 0.3888692286018281 & 0.033270694622690562\end{array}$ $\begin{array}{lll}\text { ecoli serine biosynthesis } & 0.3888692286018281 & 0.033270694622690561 \\ \text { bsub serine biosynthesis } & 0.3949156144452378 & -0.194217162462511 \\ \text { hin serine biosynthesis } & 0.37827221720278448 & -0.77862643050720692\end{array}$ 
agro serine biosynthesis

yeast L-serine degradation
vcho L-serine degradation

ecoli L-serine degradation

agro L-serine degradatio

hpy I-serine degradation

vcho peptidoglycan biosynthesis

ecoli peptidoglycan biosynthesi

hin peptidoglycan biosynthesis

0.47410518290868547

0.56530897124060242
0.57484295909879479

0.17000968576725695

0.34367292457113752

0.5325625014782619 $\begin{array}{ccc}.53256250147804785 & -0.52463905129837296 \\ .57818451218978484 & -0.35151668942337344 \\ \mathrm{~s} \quad 0.28051945669369621 & -0.914096581222\end{array}$

0.34203013654837122

0.40217206137553435

0.40249448500924423

agro peptidoglycan biosynthesis

0.49087196592541021

0.55610278145531367

0.57501996629622265

0.037810288721164244

hpy peptidoglycan biosynthesis

yeast histidine biosynthesis 0.23328485574945432

$\begin{array}{ll}\text { ecoli histidine biosynthesis } & 0.40789170664632601 \\ \text { bsub histidine biosynthesis } & 0.36449173448688266\end{array}$

hin histidine biosynthesi

tbrv histidine biosynthesis

yeast glycerol metabolism

ecoli glycerol metabolisn

bsub glycerol metabolism

hin glycerol metabolism

mtbrv glycerol metabolism

yeast tyrosine biosynthesis

ecoli tyrosine biosynthesis

bsub tyrosine biosynthesis

hin tyrosine biosynthesis

agro tyrosine biosynthesis

0.53346858121125906

0.20549952823558804

0.36482610991266512
0.3799405723649979

0.41009818801630155

0.4618616329914853

0.5146444231638152
0.60730452625338804

0.60730452625338804
0.24264817712469613

0.24264817712469613
0.2667858131293947

0.29686957992465524

0.40879438606467144
0.38951255392791667

0.50549212183081416

veho lipid-A-precursor bio 0.5966216
ynthesis $018126 \quad 0.42178482185207838$ 0.60365626627390279

0.33977097281781382

0.46323249397636818

$\begin{array}{lll}\text { hpy lipid-A-precursor biosynthesis } & 0.59128015169950288 \\ \text { vcho } & 0.19774894640349827 & \\ \text { ppgp metabolism } & 0.29199822767476941 & -0.76698257681525261\end{array}$

agro lipid-A-precurs

$\begin{array}{lll}\text { hpy lipid-A-precursor biosynthesis } & 0.59128015169950288 \\ \text { vcho ppGpp metabolism } & 0.29199822767476941 & -0.76698257681525261\end{array}$

ecoli ppGpp metabolism $0.35336875843386345 \quad-0.42030812164003128$

bsub ppGpp metabolism $0.37366122011682118 \quad-0.5505990769156140$

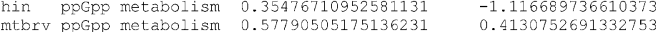

mpy serine biosynthesis

bsub peptidoglycan biosynthesis

agro histidine biosynthesis 0.53346858121125906

agro glycerol metabolisn

0.39304203259987536
$-0.43024853543420855$

.48424608465763302

.65106645978662459 785546543076651 (1) 4272057208 9519691645136701 585344893933425 .09733148181826870 98438216370834 07838 .
$-0.30883571526859588$ mtbry salvage pathways of pyrimidine deoxyribonucleotides 0.5221/303/2424/bbb

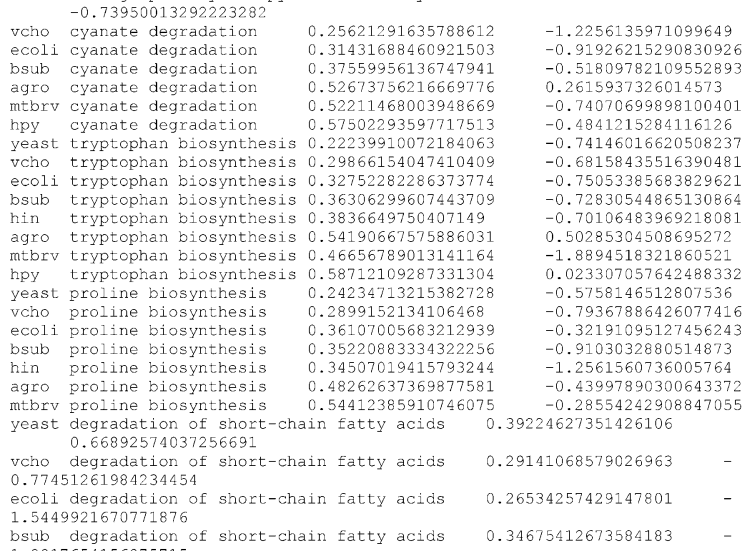

bsub degradation of short-chain fatty acids 0.34675412673584183

0.41711898590782748

vcho polyisoprenoid biosynthesis 0.30759117607959474

$-0.56714053064772751$

ecoli polyisoprenoid biosynthesis $\quad 0.29170475431732124$

bsub polyisoprenoid biosynthesis 0.34918345487987174

agro polyisoprenoid biosynthesis 0.51181745295363745

mtbrv polyisoprenoid biosynthesis 0.42580122239501689

$-0.96103150701527951$

0.024294745479938564

$-2.7325338357846518$

hpy polyisoprenoid biosynthesis 0.59262569065415982

yeast mannose catabolism

mtbrv mannose catabolism

vcho KDO biosynthesis --

ecoli KDO biosynthesis --

0.33626435568007812
KDO biosynthesis --

.356882726869891

agro KDO biosynthesis --
0.46685372583961043

0.29292258233645052

0.34083319017616076

0.36630056347728451

$\begin{array}{ll}0.36630056347728451 & -0.6740193386286385 \\ 0.4573494168369808 & -0.84199992028132942 \\ 0.49878924785158479 & -1.2230925653201263\end{array}$

2230925653201263

uding transfer to lip
-0.75518462091777727

-0.75518462091777727
uding transfer to lipid IV $<$ SUB $>A</ S U B>$
-0.63884592458281386

cluding transfer to lipid IV $<$ SUB $>A</$ SUB $>$

$-1.0862617716072771$

-1 ding transfer to lipid IV<SUB $>A</ S U B>$
-0.69083721159897726 yeast polyisoprenoid biosynthesis 0.3619221862297739 ecoli mannose catabolisn

bsub mannose catabolisn

$\begin{array}{ll}\text { hpy } & \text { KDO biosynthesis -- including transfer to lipid IV }<\text { SUB }>A</ S U B> \\ 0.57619890569780263 & -0.43479825894972129\end{array}$

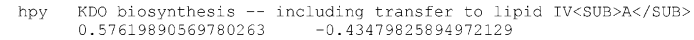

vcho menaquinone prosynthesis

ecoli menaquinone biosynthesis

bsub menaquinone biosynthesis

hin menaquinone biosynthesis

mtbrv menaquinone biosynthesis

yeast phenylalanine biosynthesis

ecoli phenylalanine biosynthesis

bsub phenylalanine biosynthesis

hin phenylalanine biosynthesis

vcho L-alanine degradation
ecoli L-alanine degradation

bsub I-alanine degradation

hin L-alanine degradation

$\begin{array}{lll}\text { agro L-alanine degradation } & 0.45531287835247203 \\ \text { mtbrv } & \text { L-alanine degradation } & 0.49544644381915848\end{array}$

py L-alanine gradion 0.60422243819959975

vcho threonine bios

ecoli threonine bios

bsub threonine biosynthesis from homoserine

0.02548772753611858

hin threonine biosynthesis from homoserine

agro threonine biosynthesis from homoserine
0.2910332744003095

2.0644985601050592

hpy threonine biosynthesis from homoserine

yeast trehalose degradation, low osmolarity

0.87518728547078395

vcho trehalose degradation, low osmolarity

ecoli trehalose degradation, low osmolarity

1.0052027054458355

bsub trehalose degradation, low osmolarity

ecoli thiamin biosynthesi

0.1997688987785110

0.25495493131881619

.38329779971414774

0.55173276317473785
0.59374727274369077

hin thiamin biosynthes

agro thiamin biosynthesis

0.59374727274369077

ecoli pyridine nucleotide cycling 0.3152864200836167 yeast thiamin biosynthesis

.45791849665959566

atho thiamin blosyrthesis

.5105273732225879

1.3241942009723702
-0.56464220367005522

$-0.4470909921065769$

0.58494001751469293

$-0.32924612064145453$

$-3.4132700849622726$

32700849622726
0.26781178977547709

0.31693069396354162 0.39933574474536693 0.62655298577918794

$-0.81594124986278271$
.1098102206553766

-0.166676271044504
0.21642790345612239

.63193830438950915

0.30748999669447347 0.34513241382872772 0.37370450821695178 0.34506706187850522 0.51180814359701099 
bsub pyridine nucleotide cycling 0.38802887759031224

agro pyridine nucleotide cycling 0.4616820337364374

vcho glycogen biosynthesis

ecoli glycogen biosynthesis

bsub glycogen biosynthesis
hin glycogen biosynthesis

agro glycogen biosynthesis

mtbrv glycogen biosynthesis

.26259967932026823

.3538075294918002

0.34117751956864517

0.45328036708129077

0.16756589234142572

0.27359228672394026

0.29336464686311403

0.33076215547798304

0.50341509292896924

0.51424662739687088

0.58810540070675354
$-0.30968849890324546$

$-0.77309112008331038$

$-1.1437596881728691$

0.41470207596999692

$-0.0952715184378641$

.90671661564293704 2810981939468849

$-1.1967870523740338$

$-1.002876444078616$

$-1.1869625847608283$

$-1.2699117747162596$

$-0.10934177787330682$

$-0.90342359944608852$

0.064591522412831406

yeast pantothenate
1.1829194176357456

vcho pantothenate and coenzyme A biosynthesis 0.2831088957028699

0.88090986267589588 and coenzyme A biosynthesis 0.32472816901625495

0.78624030742917239
bsub pantothenate and coenzyme A biosynthesis 0.36972614826095757
0.61658063563981169
hin pantothenate and coenzyme A biosynthesis 0.39426957180041927

hin pantothenate and coenzyme A biosynthesis 0.39426957180041927
0.54854373280149971 and coenzyme A biosynthesis 0.42880206524723802
agro pantothenate and

1.2960352291146744
mtbrv pantothenate and coenzyme A biosynthesis 0.53456364515221411
0.48325405929426657

hpy pantothenate and coenzyme A biosynthesis 0.56921871415696224

yeast methylglyoxal metabolism

0.21927273764675614

$-0.7674210247077603$

vcho methylglyoxal metabolism

0.31518027019641442

$-0.4698773282379866$

ecoli methylglyoxal metabolism

0.33862932885700919

$-0.60862937451995069$

bsub methylglyoxal metabolism

0.33840121828826608

$-1.1418233204575494$

agro methylglyoxal metabolism

0.43438881913369159

$-1.2071799117962083$

yeast biotin biosynthesis

vcho biotin biosynthesis

ecoli biotin biosynthesis

hin biotin biosynthesis

agro biotin biosynthesis

mtbrv biotin biosynthesis

0.18262596579645649

$-1.0717304205573903$

.2569033328513815

0.30370892401380484
0.34635234228990075
0.3216636122082227

0.4489777487390581

0.448977487390581
0.616099025258803
0.5610541778604865

$-1.2167650944928954$

$-1.054796870816627$

$-1.5928023288417503$

$-0.97514821758379566$

1.2135175164851131
-1.070008059801018

vcho pyridoxal 5'-phosphate salvage pathway 0.29945005087055399

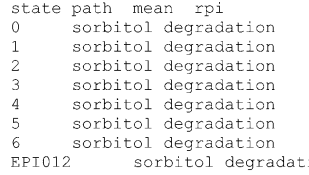

2.1630250000000002
1.9057250000000001

$\begin{array}{lr}1.77945 & 2.4501 \\ 2.1045750000000001\end{array}$

2.1045750000000001
1.92894999999999999

$\begin{array}{ll}1.1045750000000001 & 4.1399698843152128 \\ 1.928949999999999 & 3.8263259723969418 \\ 1.2572749999999999 & 2.3592046054477342 \\ 0.7971249999999997 & 1.640939137823903\end{array}$

3.2469106019372029

528488

2.3592046054477342

$2.1630250000000002 \quad 3.7031622157309503$

EPI456 sorbitol degradation $1.9289499999999999 \quad 3.8263259723969418$

EPI sorbitol deg

CAI sorbitol degradation

0 sorbitol degradation

mannitol degradation

mannitol degradatio

mannitol degradation

2.1045750000000001

5 mannitol degradation

2.1630250000000002

3.1451292784085365

3.7031622157309503
3.2469106019372029

$\begin{array}{lll}1.77945 & 2.4501369855284887 \\ 2.1045750000000001 & 4.1399698843152128\end{array}$

.104575000000000

. 1399698843152128

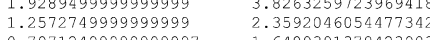

$\begin{array}{llll}6 & \text { mannitol degradation } 0.79712499999999997 & 1.6409391378423903 \\ \text { EPI012 mannitol degradation } 2.1630250000000002 & 3.7031622157309503\end{array}$

EPI456 mannitol degradation $1.9289499999999999 \quad 3.8263259723969418$

EPI mannitol degradation $2.1045750000000001 \quad 4.1399698843152128$

CAI mannitol degradation $0.69044563032330708 \quad 3.1451292784085365$

$\begin{array}{lll}\text { glycolysis } & 2.0908125000000002 & 3.5324560447449 \\ \text { glycolysis } & 1.8669312499999999 & 3.1508816618528082\end{array}$

$\begin{array}{lll}\text { glycolysis } & 1.8669312499999999 & 3.150881661852808 \\ \text { glycolysis } & 1.7679125000000002 & 2.424052577281324\end{array}$

glycolysis $2.0054249999999998 \quad 3.8807161311070684$

glycolysis $1.7046124999999999 \quad 3.2386631349802442$

glycolysis $1.47961875 \quad 3.0346316634098125$

6 glycolysis $0.90865625000000005 \quad 2.08657347587770302$

$\begin{array}{llll}\text { EPI012 } & \text { glycolysis } & 2.0908125000000002 & 3.5324560447449 \\ \text { EPI456 } & \text { glycolysis } & 1.7046124999999999 & 3.2386631349802442\end{array}$

$\begin{array}{lrrr}\text { EPI456 glycolysis } & 1.7046124999999999 & 3.2386631349 \\ \text { EPI } & \text { glycolysis } & 2.0054249999999998 & 3.8807161311070684\end{array}$

$\begin{array}{llll}\text { CAI } & \text { glycolysis } & 0.73216433931129328 & 3.8807161311070684 \\ 0 & \text { gluconeogenesis } 1.4417705882352942 & 1.9981585260768202\end{array}$

$\begin{array}{llll}0 & \text { gluconeogenesis } & 1.4417705882352942 & 1.9981585260768202 \\ 1 & \text { gluconeogenesis } & 1.4030999999999998 & 2.0027270411853069\end{array}$

gluconeogenesis 1.3892705882352941 .5680048887909686

gluconeogenesis $1.4324882352941177 \quad 2.3826222672550172$

gluconeogenesis $\quad 1.1873588235294119 \quad 1.883692329034184$

gluconeogenesis $\quad 1.2368294117647056 \quad 2.297095805402448$

$\begin{array}{lllll}6 & \text { gluconeogenesis } & 1.1611 & 3.0952378943447876 & \\ \text { EPI012 gluconeogenesis } & 1.403099999999998 & 2.0027270411853069\end{array}$

$\begin{array}{llrr}\text { EPI012 } & \text { gluconeogenesis } & 1.4030999999999998 & 2.0027270 \\ \text { EPI456 } & \text { gluconeogenesis } & 1.1611 & 3.0952378943447876\end{array}$

$\begin{array}{llll}\text { EPI456 gluconeogenesis } & 1.1611 & 3.09523789434 \\ \text { EPI gIuconeogenesis } 1.1611 & 3.0952378943447876\end{array}$

$\begin{array}{lllll}\text { EPI } & \text { gluconeogenesis } & 1.1611 & 3.0952378943447876 \\ \text { CAI } & \text { gluconeogenesis } & 0.60472923305046022 & 2.4333529403709035 \\ 0 & \text { glycine cleavage } & 1.1002000000000001 & 1.1907054225407312\end{array}$

glycine cleavage 1.1404000000000001

glycine cleavage 1.620850000000000

glycine cleavage 1.1103499999999999

glycine cleavage 1.6384000000000001

0.48739999999999999

EPI012 glycine cleavage 0.41794999999999999

EPI456 glycine cleavage 1.638400000000000

$\begin{array}{lll}\text { EPI glycine cleavage } & 1.6384000000000001 \\ \text { CAI glycine cleavage } & 0.4507795480917719\end{array}$

1.352446968746565

2.091568218637887

1.5403071230104579

3.0652163165818358
0.02050875162429644

.12590725104578002
2.091568218637887

2.0915682186378879
3.0652163165818358

3.0652163165818358
1.1549774312703325 ecoli pyridoxal 5'-phosphate salvage pathway

hin pyridoxal $5^{\prime}$-phosphate salvage pathway
1.6587487576688345 agro pyridoxal 5 '-phosphate salvage pathway
0.21726450954684534

0.21726450954684534
mtbrv pyridoxal 5'-phosphate salvage pathway
1.1258764961765457

vcho enterobacterial common antigen biosynthesis

vcho enterobacteri
0.66957948627551034

ecoli enterobacterial common antigen biosynthesis
0.70177101667232555

bsub enterobacterial common antigen biosynthesi

1.2049460713002753

hin enterobacterial common antigen biosynthesis
1.0870777347848799

agro enterobacterial common antigen biosynthesis

mtbrv enterobacterial common antigen biosynthesis
0.62286922792370836

0.62286922792370836
hpy enterobacterial common antigen biosynthesis

yeast methionine

vcho methionine biosynthesis from homoserine 0.27152809748722179

1.029331466569112
ecoli methionine biosynthesis from homoserine 0.30250196687085623
1.0702177987533081

bsub methionine bios
0.77321455703579745

作

hin methionine biosynthesis from homoserine 0.39089559030602639

agro methionine biosynthesis from homoserine 0.48601588436937482

0.3860699511693616 frosynthesis from homoserine 0.49891967582609154

mtbrv methionine biosynthesis from homoserine 0.49891967582609154
1.2203952274169283
hpy methionine biosynthesis from homoserine 0.53563056391125796

2.136341435156782

vcho cobalamin biosynthesis 0.2611752885226885

$\begin{array}{lll}\text { ecoli cobalamin biosynthesis } & 0.25900787324509139 & -1.625928734632597 \\ \text { agro cobalamin biosynthesis } & 0.51723138061436236 & 0.1104013236485899 \\ \text { mtbry cobalamin biosynthesis } & 0.47447518404691474 & -1.725923695904905\end{array}$

ecoli dTDP-rhamnose biosynthesis $0.29762877087733919^{-1.7259236959049056}$

hin dTDP-rhamnose biosynthesis $\quad 0.3568259939976639$

$-1.1324811563848451$

agro dTDP-rhamnose biosynthesis $\quad 0.3922520448439869$

$-1.0870777347848799$

mtbrv dTDP-rhamnose biosynthesis $\quad 0.51875480275012031$

vcho enterobactin biosynthesis $\quad 0.23273099368631767$

$-1.8773501978663889$

ecoli enterobactin biosynthesis

0.28701579326090176

$-0.8101915125370522$

bsub enterobactin biosynthesis $\quad 0.36565126874464915$

1.268079975259937

agro enterobactin biosynthesis $\quad 0.45431638048987844$

$-0.68490642788645084$

mtbrv enterobactin biosynthesis $\quad 0.43198478130141438$

$-0.8902391938632784$

$-2.6046536944425771$
0
1
2
3
4
5
6
$\mathrm{EP}$

$\mathrm{EP}$
$\mathrm{EP}$
$\mathrm{CA}$
0
1
2
3
4
5
6
$\mathrm{EP}$

pyruvate dehydrogenase 1.0300666666666667

pyruvate dehydrogenase 0.89216666666666666

pycuvate dehyarogenase 0.91246666666666654

pyruvate dehydrogenase 1.0442833333333335

pyruvate dehydrogenase 0.9893833333333335

pyruvate dehydrogenase 0.93806666666666672 EPI012 pyruvate dehyarogenase 0.722833333333333446

EPI456 pyruvate dehydrogenase 0.93806666666666672

1.0249142812311012

0.73797726953944487

0.49002888497340485

1.365084463056205

1.389526143785053

3440993041950535

PI pyruvate dehydrogenase 0.93806666666666672

pyruvate dehydrogenase 0.31644122380609985

glutamine biosynthesis 0.82709999999999995

glutamine biosynthesis 1.6095999999999999

glutanine biosynthesis 1.009500000000001

glutamine blosynthesis 0.7660000000000000

glutamine biosynthesis 0.51419999999999999

glutamine biosynthesis 0.56379999999999997

EPI 456 glutamine biosynthesis 1.609599999999999

EPI

gutamine biosynthesis 1.1821999999999999

glutamine biosynthesis 1.60959999999999999

$\begin{array}{lll}\text { glutamine biosynthesis } & 1.6095999999999999 & 2.5138912397334625 \\ \text { glutamine biosynthesis } & 0.54953273363146293 & 1.9750093389256329 \\ \text { thioredoxin pathway } & 1.5944 & 2.3589656226626761 \\ \text { thioredoxin pathway } & 1.0103 & 1.0304011468041196\end{array}$

thioredoxin pathway

$\begin{array}{lll}\text { thioredoxin pathway } & 0.53390000000000004 & -0.3658486866063668 \\ \text { thioredoxin pathway } & 0.55130000000000001 & 0.07852385794729546\end{array}$

2.3589656226626761

thloredoxin pathway

0.53390000000000004

$\begin{array}{ll}0.55130000000000001 & 0.078523857947295467 \\ 0.52890000000000004 & 0.15882787009461979\end{array}$

.6830000000000001

3.6524551881643874

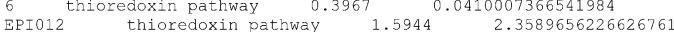

$\begin{array}{lllll}\text { EPI012 } & \text { thioredoxin pathway } & 1.5944 & 2.3589656226626761 \\ \text { EPI456 } & \text { thioredoxin pathway } & 1.6830000000000001 & 3.6524551881643874\end{array}$

EPI thioredoxin pathway $\quad 1.6830000000000001 \quad 3.6524551881643874$

CAI thioredoxin pathway $0.33934484224977524 \quad 0.22964004684851869$

0 non-oxidative branch of the pentose phosphate pathway 1.2160466666666667

1.4645602383712859 .

non-oxidative branch of the pentose phosphate pathway 1.0838266666666669

1.212406956191936

non-oxidative branch of the pentose phosphate pathway 1.0551799999999998

0.81268048618513633
non-oxidative branch of the pentose phosphate pathway 0.76809999999999989

0.64540448019162688 . 0.7680998999999989

non-oxidative branch
0.80349861969399228

hon-oxidative branch of the pentose phosphate pathway 0.6781133333333334

0.59985017969445631

6 non-oxidative branch of the pentose phosphate pathway 0.60205999999999993

EPI012 non-oxidative branch of the pentose phosphate pathway

EPI456 non-oxidative branch of the pentose pho

non-oxidative branch of the pentose phosphate pathway

EPI non-oxidative branch of the pentose phosphate pathway 1.216046666666667

CAI non-oxidative branch of the pentose phosphate pathway 0.42565117193895424

0.94631509678196291 


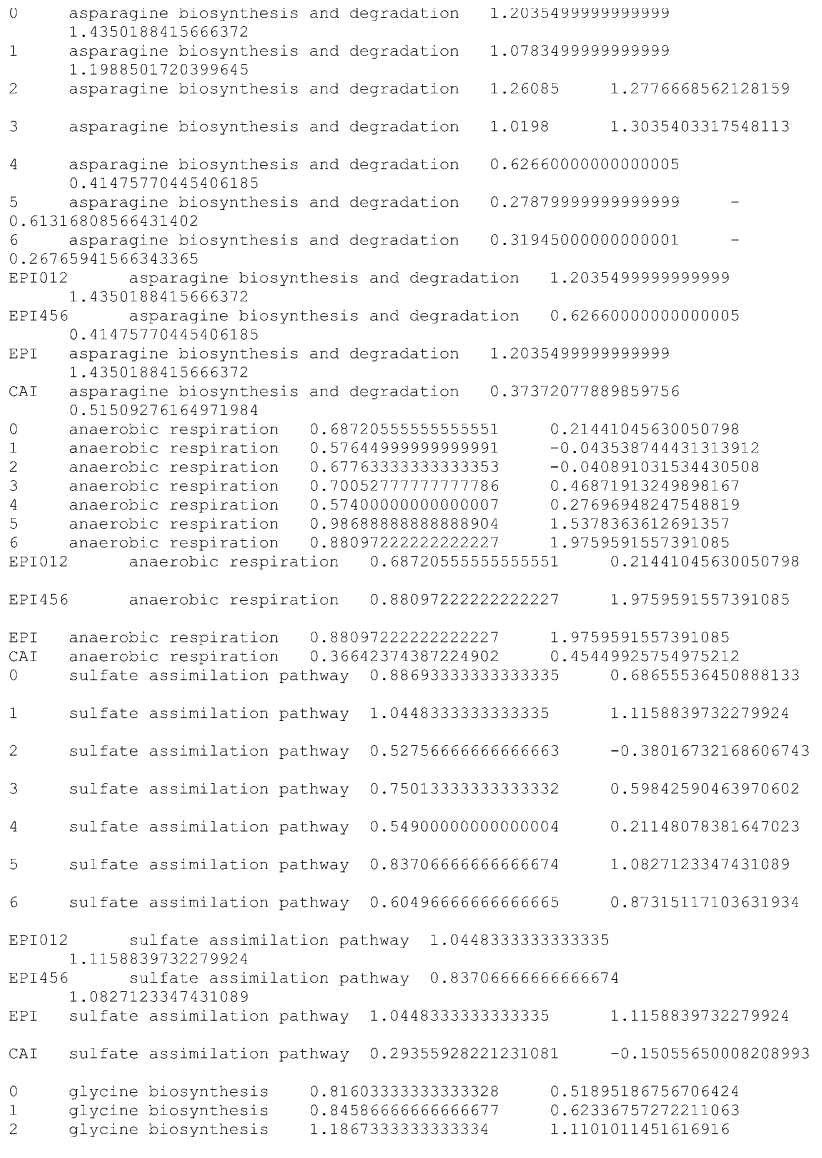

degradation of short-chain fatty acids

degradation of short-chain fatty acids

degradation of short-chain fatty acids

$0.9022 \quad 0.46681762389683834$

0.62090000000000001

degradation of short-chain fatty acids

$1.0183 \quad 1.4408346350435548$

$0.2893-0.581271600565894$

degradation of short-chain fatty acids

0.2268

$-0.63785181841072969$

EPI012 degradation of short-chain fatty acids 1.3125

EPI456 degradation of short-chain fatty acids 1.0183

EPI degradation of short-chain fatty acids $1.3125 \quad 1.7784583903937199$

CAI degradation of short-chain fatty acids 0.39224627351426106

0.66892574037256558 . 0.77249999999999996

$\begin{array}{ll}\text { UDP-N-acetylglucosamine biosynthesis } & 0.77249999999999996 \\ 0.4160415868722753 & \\ \text { UDP-N-acetylglucosamine biosynthesis } & 0.76449999999999996 \\ 0.42195485138941519\end{array}$

0.42195485138941519
UDP-N-acetylglucosamine biosynthesis

0.7941868385611448

1.0469999999999999

0.024659739782012861 ine biosynthesis

UDP-N-acetylglucosamine biosynthesis

0.29792586604637367

UDP-N-acetylglucosamine biosynthesis

0.53069999999999995

0.58199999999999996

$0.4325-0.14626429903315655$

0.52590000000000003

UDP-N-acetylglucosamine biosynthesis

EPI012 UDP-N-acetylglucosamine biosynthesis
0.7941868385611448

0.7941868385611448
UDP-N-acetylglucosamine biosynthesis

0.55723234415501499

EPI UDP-N-acetylglucosa 0.79418683856114
UDP-N-acetylglu
to35521168017

0.1263503552116801

\begin{tabular}{|c|c|c|c|}
\hline lose & biosynthesis & 0.44127142857142854 & -0.36696358742124841 \\
\hline trehalose & biosynthesis & 0.31695714285714283 & -0.68587994709668632 \\
\hline trehalose & biosynthesis & 0.47577142857142857 & -0.4972679184995264 \\
\hline trehalose & biosynthesis & 0.80674285714285709 & 0.74644639394827683 \\
\hline trehalose & biosynthesis & 0.51038571428571433 & 0.11032881097342712 \\
\hline trehalose & biosynthesis & 1.0128571428571427 & 1.6167216970529823 \\
\hline trehalose & biosynthesis & 0.74698571428571425 & 1.4406025738855139 \\
\hline trel & 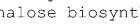 & esis $\quad 0.44127142857142854$ & $4 \quad-0.36696358742124841$ \\
\hline tre & alose biosy & 1.0128571428571427 & 1.6167216970529823 \\
\hline
\end{tabular}

EPI456 trehalose biosynthesis $1.0128571428571427 \quad 1.6167216970529823$

EPI trehalose biosynthesis $1.0128571428571427 \quad 1.6167216970529823$

CAI trehalose biosynthesis $0.2240587114615448 \quad-0.72767900306176592$

0.038449926822698127

0.038449926822698127
removal of superoxide radicals $\quad 0.38302000000000003$

2 removal of superoxide radicals $\quad 0.69485999999999992$ $\begin{array}{llll}3 & \text { gLycine biosynthesis } & 0.798599999999999998 & 0.125154 / 522324 b / 96 \\ 4 & \text { glycine biosynthesis } & 1.1485333333333334 & 1.7819870959245989\end{array}$

glycine biosynthesis $\quad 0.37319999999999998 \quad-0.326403495827090$

$\begin{array}{lllll}6 & \text { glycine biosynthesis } & 0.3246 & -0.24708207217559155 & \\ \text { EPI012 } & \text { glycine biosynthesis } & 1.1867333333333334 & 1.1101011451616916\end{array}$

EPI456 glycine biosynthesis $1.1485333333333334 \quad 1.7819870959245989$

$\begin{array}{llll}\text { EPI } & \text { glycine biosynthesis } & 1.14853333333333334 & 1.7819870959245989 \\ \text { CAI } & \text { Glycine biosynthesis } & 0.37859998542652501 & 0.55560897401364817\end{array}$

$\begin{array}{llll}\text { CAI } & \text { Glycine biosynthesis } & 0.37859998542652501 & 0.55560 \\ 0 & \text { TCA cycle, aerobic respiration } & 0.36978461538461538\end{array}$

0.53595427677719021
TCA cycle, aerobic respiration $\quad 0.30456153846153844$

1. TCA cycle, aerobic respiration
0.7165636719702897
2.

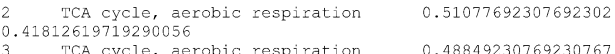

3 TCA cycle, aerobic respiration

.085703373127958976
TCA cycle, aerobic respiration $\quad 0.53179999999999994$

0.16642455913906562
TCA Cycle, aerobic respiration $\quad 1.0865307692307695$

1.8405245280789777
TCA cycle, aerobic respiration $\quad 1.1963461538461539$

$6 \quad$ TCA cycle, aerobic respiration

EPI012 TCA cycle, aerobic respiration 0.51077692307692302

0.41812619719290056
EPI456 TCA cycle, aerobic respiration 1.1963461538461539
3.2360674505229694

EPI TCA CYCle, aerobic respiration 1.1963461538461539

CAI TCA cycle, aerobic respiration 0.26888666616141943

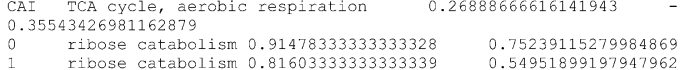

ribose catabolism 0.8325666666666666

ribose catabolism $0.6005166666666667 \quad 0.20721377780334654$

ribose catabolism $0.48015000000000002 \quad 0.031124907709534792$

ribose catabolism $0.66353333333333331 \quad 0.55555963181493551$

$\begin{array}{lrll}6 & \text { ribose catabolism } 0.63748333333333329 & 1.0030747863915943 \\ \text { EPI012 } & \text { ribose catabolism } 0.91478333333333328 & 0.75239115279984869\end{array}$

$\begin{array}{lll}\text { EPI012 } & \text { ribose catabolism } 0.914783333333333328 & 0.75239115279984869 \\ \text { EPI456 } & \text { ribose catabolism } 0.63748333333333329 & 1.0030747863915943\end{array}$

$\begin{array}{lll}\text { EPI ribose catabolism } & 0.63748333333333329 & 1.0030747863915943 \\ \text { CAI ribose catabolism } & 0.31473803493381353 & 0.025308743161060788\end{array}$

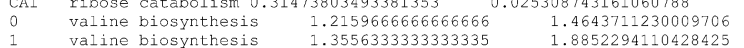

$\begin{array}{lll}\text { valine biosynthesis } & 1.2159666666666666 & 1.4643711230009706 \\ \text { valine biosynthesis } & 1.35633333333335 & 1.885229410428425 \\ \text { valine biosynthesis } & 0.80003333333333337 & 0.23583543169009352\end{array}$

$\begin{array}{lll}\text { valine biosynthesis } & 0.80003333333333337 & 0.23583543169009352 \\ \text { valine biosynthesis } & 0.5120000000000001 & -0.0242363286957832\end{array}$

$\begin{array}{lll}\text { valine biosynthesis } & 0.62443333333333328 & 0.40908201723694676 \\ \text { valine biosynthesis } & 0.2936000000000003 & -0.56820923047796956\end{array}$

$\begin{array}{lll}\text { valine biosynthesis } & 0.2936000000000003 & -0.56820923047796956 \\ \text { valine biosynthesis } & 0.27783333333333332 & -0.4339429971816921\end{array}$

EPI012 valine valine biosynthesis $1.35563333333333355^{-0.4339429971816921} 1.8852294110428425$

EPI456 valine biosynthesis $0.62443333333333328 \quad 0.40908201723694676$

EPI valine biosynthesis $1.3556333333333335 \quad 1.8852294110428425$

CAI valine biosynthesis $\quad 0.50448644104551632 \quad 1.6009515600278992$ degradation of short-chain fatty acids 0.60840000000000005

degradation of short-chain fatty acids $\quad 1.3125 \quad 1.7784583903937199$

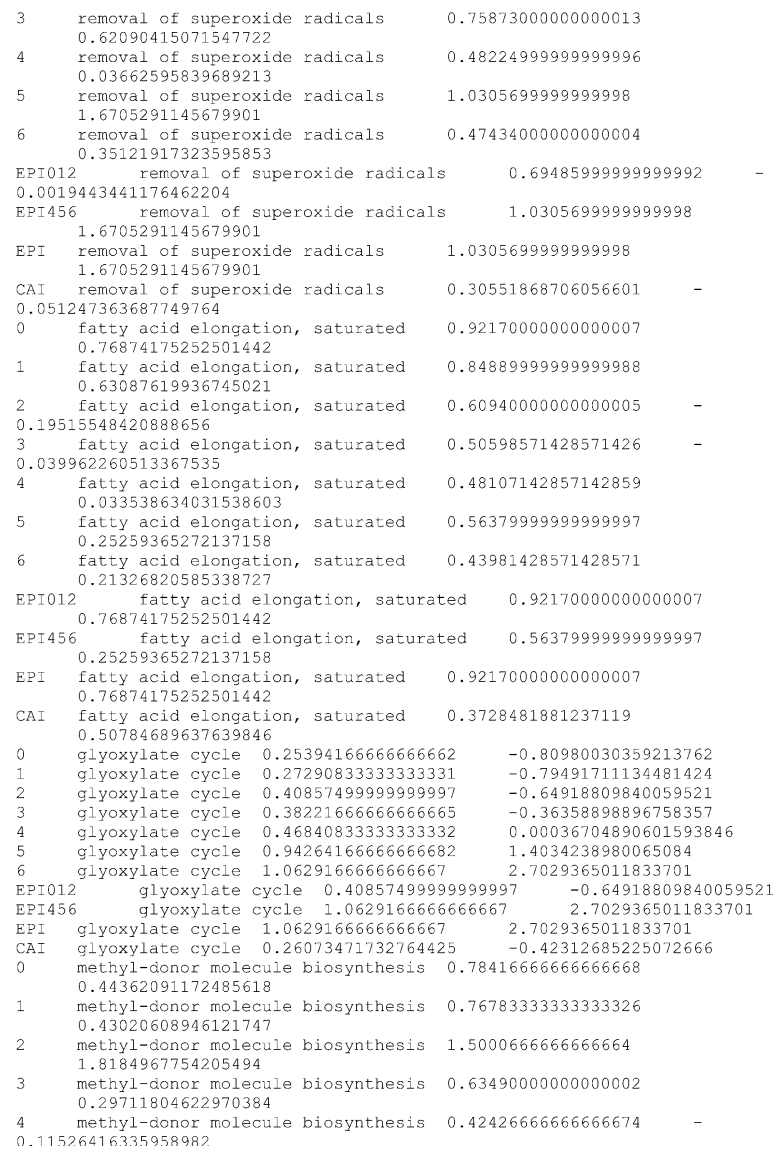




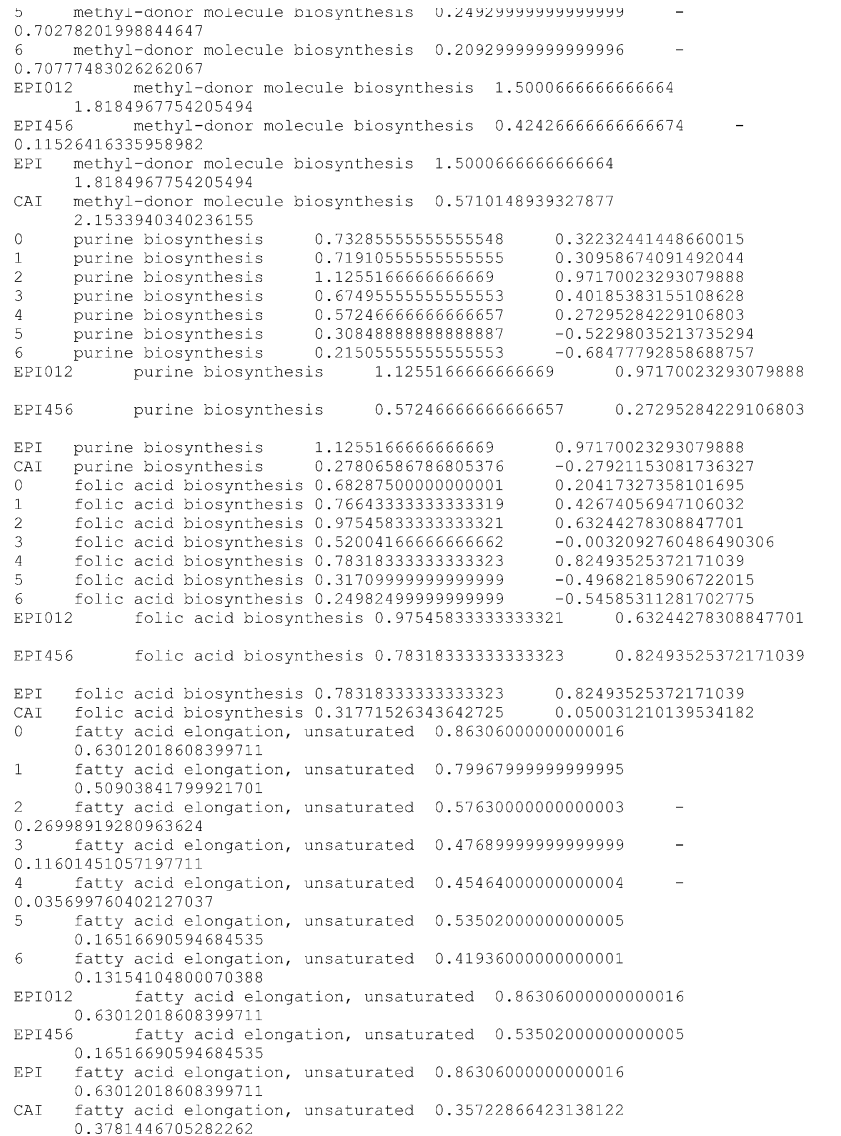

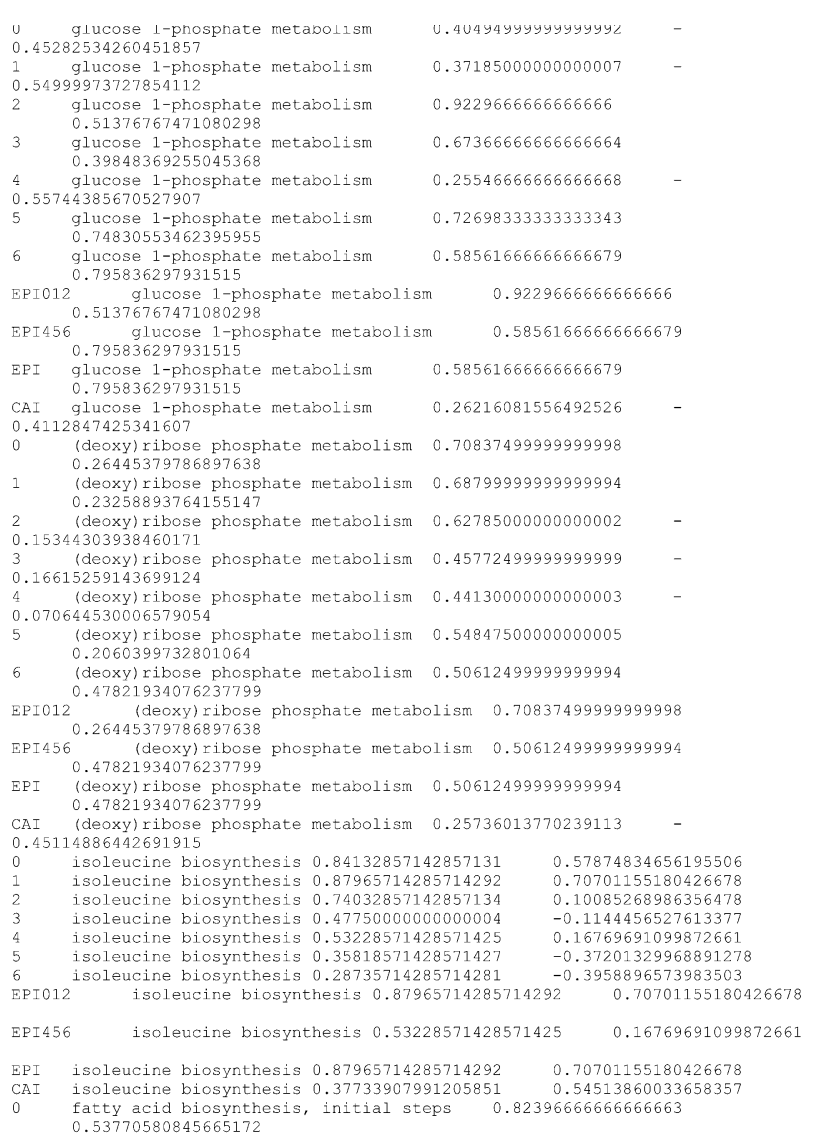

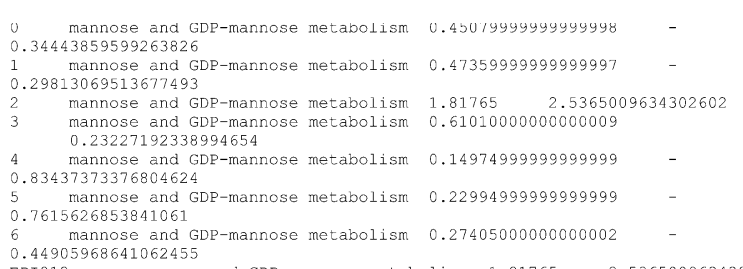

$\begin{array}{llll}0.44905968641062455 \\ \text { EPI012 mannose and GDP-mannose metabolism } & 1.81765 & 2.5365009634302602\end{array}$

EPI456_mannose and GDP-mannose metabolism 0.27405000000000002
0.44905968641062455

EPI mannose and GDP-mannose metabolism $1.81765 \quad 2.5365009634302602$

$\begin{array}{llll}C A I & \text { mannose and GDP-mannose metabolism } & 0.42187117411450392\end{array}$
0.91492655159249114
phosphatidic acid synthesis 0.51900000000000002
$-0.18321774279895819$
phosphatidic acid synthesis 0.70479999999999998
0.27417517752343534
phosphatidic acid synthesis $1.0699000000000001 \quad 0.8459600085598511$
phosphatidic acid synthesis $\quad 0.79369999999999996 \quad 0.71234241344557037$

phosphatidic acid synthesis $0.2447 \quad-0.58564765626109605$

phosphatidic acid synthesis $0.21260000000000001-0.81426782980863821$

phosphatidic acid synthesis $0.32940000000000003 \quad-0.22790318892478714$

EPI012 phosphatidic acid synthesis 1.0699000000000001

EPI456 0.8459600085598511 phosphatidic acid synthesis 0.32940000000000003

0.22790318892478714

EPI phosphatidic acid synthesis $1.0699000000000001 \quad 0.8459600085598511$

CAI phosphatidic acid synthesis $0.24426581104065304 \quad-0.55988222440112489$

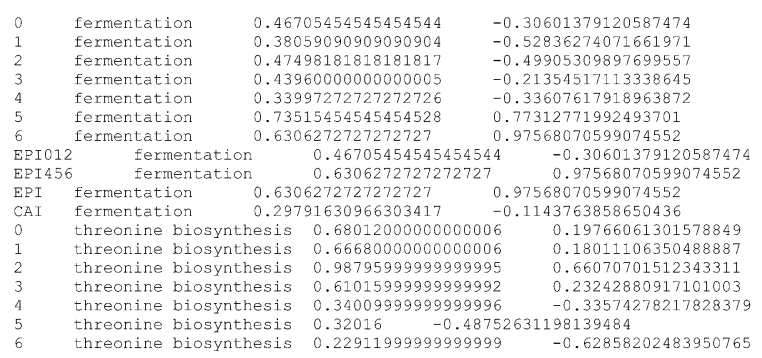




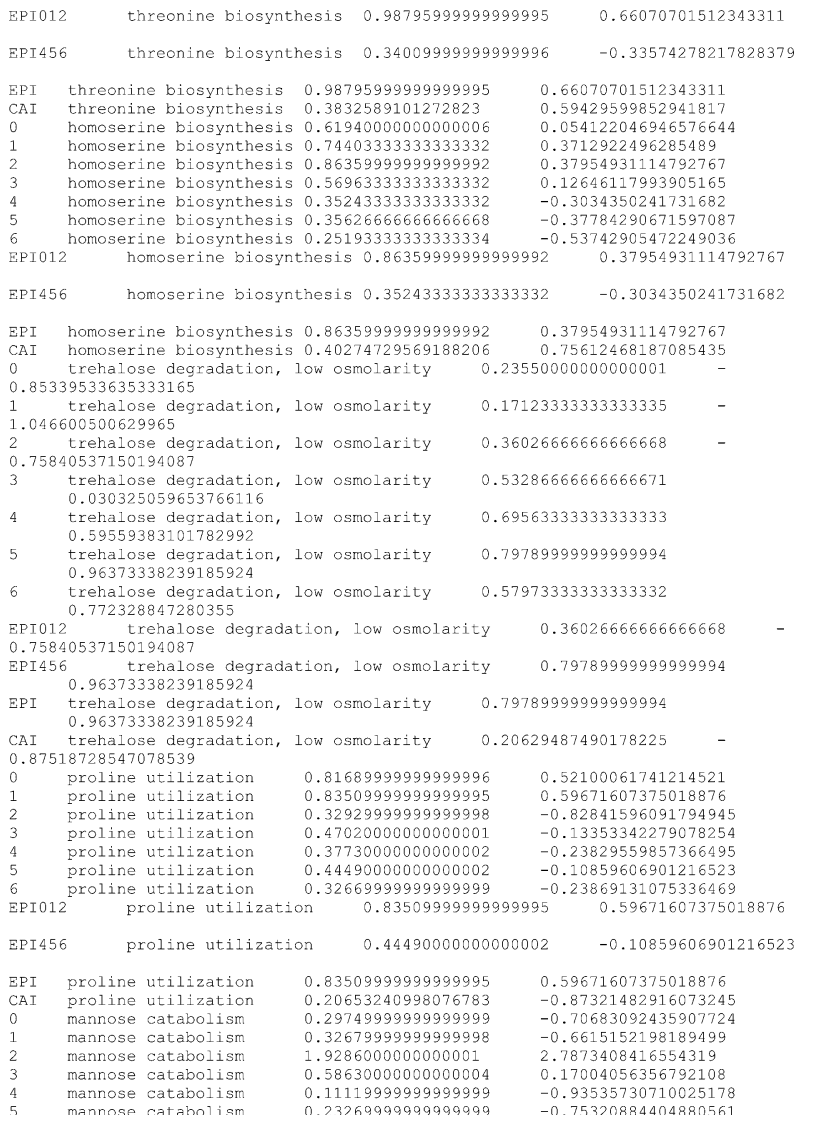

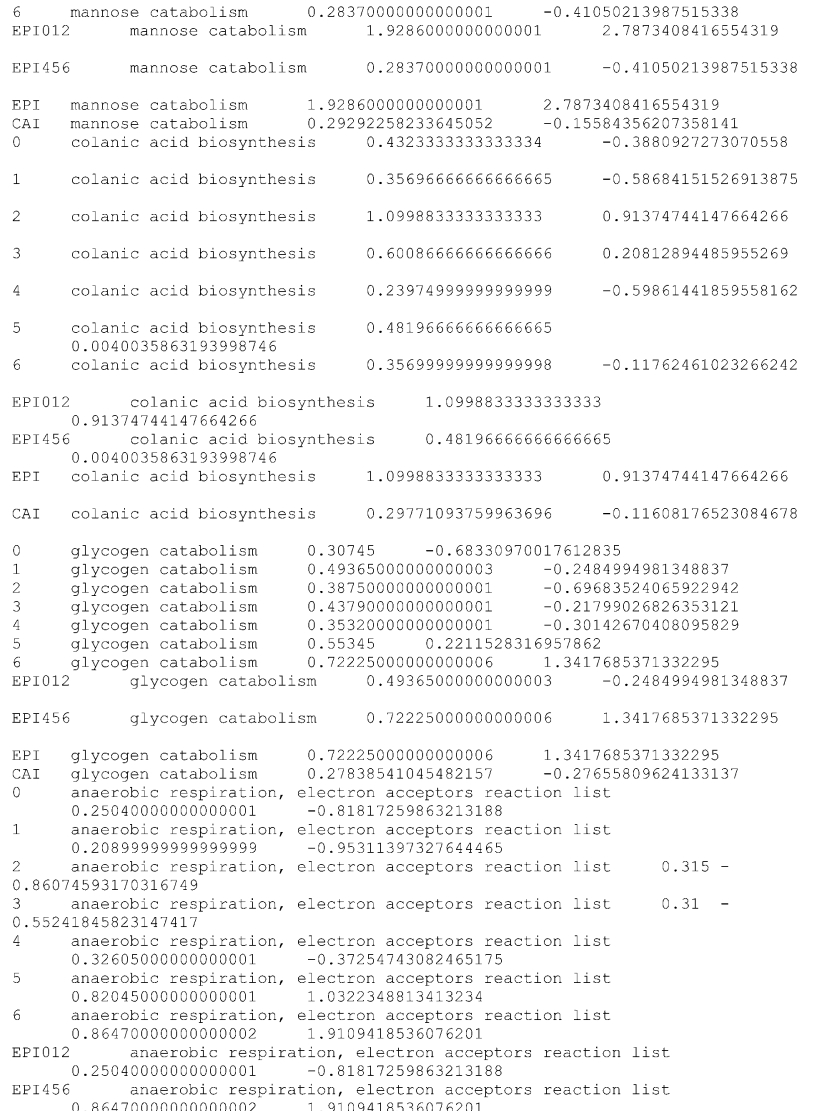
EPI mannose catabolism $1.9286000000000001 \quad 2.7873408416554319$

$\begin{array}{lll}\text { A I mannose catabolism } & 0.29292258233645052 & -0.15584356207358141 \\ \text { colanic acid biosynthesis } & 0.4323333333333334 & -0.3880927273070558 \\ \text { colanic acid biosynthesis } & 0.3569666666666665 & -0.58684151526913875 \\ \text { colanic acid biosynthesis } & 1.0998833333333333 & 0.91374744147664266 \\ \text { colanic acid biosynthesis } & 0.60086666666666666 & 0.20812894485955269 \\ \text { colanic acid biosynthesis } & 0.239749999999999999 & -0.59861441859558162 \\ \text { colanic acid biosynthesis } & 0.48196666666666665 & \\ 0.0040035863193998746 & 0.35699999999999998 & -0.11762461023266242 \\ \text { colanic acid biosynthesis } & & \end{array}$

EPI012 colanic acid biosynthesis 1.0998833333333333

EPI456 colanic acid biosynthesis 0.48196666666666665

EPI colanic acid biosynthesis $\quad 1.0998833333333333 \quad 0.91374744147664266$

CAI colanic acid biosynthesis $\quad 0.29771093759963696 \quad-0.11608176523084678$

glycogen catabolism $\quad 0.30745 \quad-0.68330970017612835$

$0.49365000000000003-0.2484994981348837$

$\begin{array}{ll}0.38750000000000001 & -0.69683524065922942 \\ 0.43790000000000001 & -0.21799026826353121\end{array}$

$0.5532000000000001-0.30142670408095829$ $6 \quad$ glycogen catabolism $\quad 0.72225000000000006 \quad 1.3417685371332295$

EPI456 gIycogen catabolism $0.72225000000000006 \quad 1.3417685371332295$

$\begin{array}{llll}\text { EPI } & \text { glycogen catabolism } & 0.72225000000000006 & 1.3417685371332295 \\ \text { CAI glycogen catabolism } & 0.27838541045482157 & -0.2765580962413313\end{array}$

anaerobic respiration, electron acceptors reaction lis

anaerobic respiration, electron acceptors reaction list

0.20899999999999999
anaerobic respiration, electron acceptors reaction list $0.315-$

3 anaerobic respiration, electron acceptors reaction list 0.31 -

anaerobic respiration, electron acceptors reaction list
0.3260500000000001
-0.37254743082465175

anaerobic respiration, electron acceptors reaction list

0.8204500000000001
anaerobic respiration, electron acceptors reaction list
0.8647000000000002
1.9109418536076201

EPI012 anaerobic respiration, electron acceptors reaction list

$\begin{array}{cc}0.25040000000000001 & -0.81817259863213188 \\ \text { EPI456 anaerobic respiration, electron acceptors reaction list } \\ n \text { n R6470nononononn? }\end{array}$
EPI anaerobic respiration, electron acceptors reaction list

AI anaerobic respiration, electron acceptors reaction list $0.24173134739194152-0.58092803724632758$

$\begin{array}{lll}0.24173134739194152 & -0.58092803724632758 & \\ \text { formy THF biosynthesis } & 0.5613416666666674 & -0.083124493156107634 \\ \text { formylTHF biosynthesis } & 0.66680833333333334 & 0.18013169160006823\end{array}$ formylTHF biosynthesis 0.8222583333333332 formylTHF biosynthesis 0.3631666666666666 formylTHE biosynthesis 0.56635000000000002 formylTHF biosynthesis 0.26704166666666668 0.28608253663425209

$-0.41340022445538122$

$-0.6488970860582858$

$-0.64888708604040179$

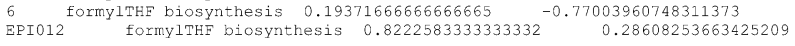

EPI456 formylTHF biosynthesis $0.56635000000000002 \quad 0.25692994068582858$

EPI formylTHE biosynthesis 0.8222583333333332

$\begin{array}{lll}\text { EPI } & \text { formylTHF biosynthesis } & 0.8222583333333332 \\ \text { CAI } & \text { formylTHF biosynthesis } & 0.2866672062266305 \\ 0 & \text { alanine biosynthesis } & 0.5589500000000006 \\ 1 & \text { alanine biosynthesis } & 0.65654999999999997 \\ 2 & \text { alanine biosynthesis } & 0.6101000000000009 \\ 3 & \text { alanine biosynthesis } & 0.33555000000000001 \\ 4 & \text { alanine biosynthesis } & 0.65949999999999998 \\ 5 & \text { alanine biosynthesis } & 0.3045499999999999 \\ 6 & \text { alanine biosynthesis } & 0.23714999999999997 \\ \text { EPI012 alanine biosynthesis } 0.65654999999999\end{array}$

0.28608253663425209

$-0.20778728395357488$

0.158773850643409645

$-0.19357289822639329$

$-0.4856112631284175$

0.50094083188932925 $-0.5349457531610459$ EPI012 alanine biosynthesis $0.65654999999999997 \quad 0.5964973514011829840 .15473850643409645$

EPT456 alanine biosynthesis $0.65949999999999998 \quad 0.50094083188932925$

$\begin{array}{llll}\text { EPI } & \text { alanine biosynthesis } & 0.65949999999999998 & 0.50094083188932925 \\ \text { CAI alanine biosynthesis } & 0.29927715769637842 & -0.1030761023145799 \\ & \text { glutate }\end{array}$ glutamate degradation $\mathrm{V} 0.62875000000000003 \quad 0.076224905852161726$ glutamate degradation V $0.47015000000000001 \quad-0.30667072654109023$ $\begin{array}{lll}\text { glutamate degradation V } & 1.05965 & 0.82278642810191482 \\ \text { glutamate degradation V } & 0.41470000000000001 & -0.27865277027491725\end{array}$ $\begin{array}{lll}\text { glutamate degradation V } 0.41470000000000001 & -0.27865277027491725 \\ \text { glutamate degradation V } 0.22520000000000001 & -0.63672884121513007\end{array}$ glutamate degradation $V 0.21494999999999997 \quad-0.80712909266756399$ 6 glutamate degradation V $0.35120000000000001 \quad-0.14079909416071754$ $\begin{array}{llll}\text { EPI012 } & \text { glutamate degradation V } 1.05965 & 0.82278642810191482 \\ \text { EPI456 } & \text { glutanate degradation V } 0.3512000000000001 & -0.14079909416071754\end{array}$ EPI glutamate degradation V $1.05965 \quad 0.82278642810191482$

CAI glutamate degradation V $0.21813134649369936 \quad-0.77689896873059894$ aspartate biosynthesis and degradation 0.62875000000000003 0.076224905852161726

1 aspartate biosynthesis and degradation 0.4701500000000000 aspartate biosynthesis and degradation $\quad 1.05965 \quad 0.82278642810191482$

3 aspartate biosynthesis and degradation 0.41470000000000001

0.27865277027491725
4 aspartate biosynthesis and degradation 0.22520000000000001
0.63672884121513007

5 aspartate biosynthesis and degradation 0.21494999999999997

6 aspartate biosynthesis and degradation 0.35120000000000001

EPI012 aspartate biosynthesis and degradation 1.05965

EPI456 aspartate biosynthesis and degradation 0.35120000000000001
EPI aspartate biosynthesis and degradation $1.05965 \quad 0.82278642810191482$

CAI aspartate biosynthesis and degradation 0.21813134649369936

$\begin{array}{llll}0.7768996873059894 & & \\ 0 & \text { cysteine biosynthesis } & 0.58919999999999995 & -0.017269005347399217 \\ 1 & \text { cysteine biosynthesis } & 0.93630000000000002 & 0.84722366161010787 \\ 2 & \text { cysteine biosynthesis } & 0.6508000000000005 & -0.10155682753000334 \\ 3 & \text { cysteine biosynthesis } & 0.503-0.047769195809167768 \\ 4 & \text { cysteine biosynthesis } & 0.31280000000000002 & -0.40725644111393122 \\ 5 & \text { cysteine biosynthesis } & 0.2369999999999999 & -0.74014647396088129 \\ 6 & \text { cysteine biosynthesis } & 0.1549000000000001 & -0.92513550710506942 \\ \text { EPI012 } & \text { cysteine biosynthesis } 0.93630000000000002 & 0.84722366161010787\end{array}$

EPI456 cysteine biosynthesis $0.31280000000000002 \quad-0.40725644111393122$

EPI cysteine biosynthesis 0.93630000000000002

$\begin{array}{llll}\text { EPI } & \text { cysteine biosynthesis } & 0.93630000000000002 & 0.84722366161010787 \\ \text { CAI cysteine biosynthesis } & 0.4715765155190786 & 1.3241942009723688 \\ & \text { cyrimidine biosynthesis } & 0.5883399999999997 & -0.0193019955782871\end{array}$

pyrimidine biosynthesis $0.53539999999999999-0.019301995578287198$

pyrimidine biosynthesis $0.65405999999999997 \quad-0.0941864985258520994$

pyrimidine biosynthesis $0.4546466666666667 \quad-0.17420170359324327$

pyrimidine biosynthesis $0.34355999999999998 \quad-0.32667914628387568$

pyrimidine biosynthesis $0.34905333333333327 \quad-0.39975528568517138$

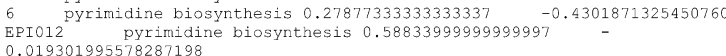

0.019301995578287198
EPI456 pyrimidine biosynthesis 0.34355999999999998

$-0.32667914628387568$

$\begin{array}{lll}\text { EPI pyrimidine biosynthesis } & 0.58833999999999997 & -0.019301995578287198 \\ \text { CAI pyrimidine biosynthesis } 0.23324451545152713 & -0.65140143967900943\end{array}$ o galactose, galactoside and glucose catabolism 0.30227999999999999

0.69553128098274597
1 galactose, galactoside and glucose catabolism 0.34585999999999995

2 galactose, galactoside and glucose catabolism 0.32816000000000006

3 galactose, galactoside and glucose catabolism 0.39648
0.32629375245799719

galactose, galactoside and glucose catabolism 0.53766000000000003 0.18177511010473965
galactose, galactoside and glucose catabolism 0.58499999999999996 galactose, galactoside and glucose catabolism 0.45038

0.25548458100902677
EPI012 galactose, galactoside and glucose catabolism 0.34585999999999995

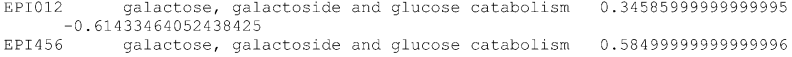
EPI456 galactose, galactoside and glucose catabolism 0.58499999999999
EPI galactose, galactoside and glucose catabolism 0.58499999999999996 $\begin{array}{ll}\text { EPI galactose, galactoside and glucose catabolism } & 0.58499999999999996 \\ 0.31699417501532429\end{array}$ CAI galactose, ga
0.73143107850978584
thiamin biosynthesis
thiamin biosynthesis
thiamin biosynthesi
thiamin biosynthesis
thiamin biosynthesis
0.52769999999999995 0.31180000000000002 0.42159999999999997
0.65739999999999998 0.361099999999999998 $-0.16265144627718395$ $-0.69864579114206038$ $-0.6197406949406324$ 0.35595021412867733 -0.2807322753047086
0.1192359674051205 $-0.5279727997863298$ 


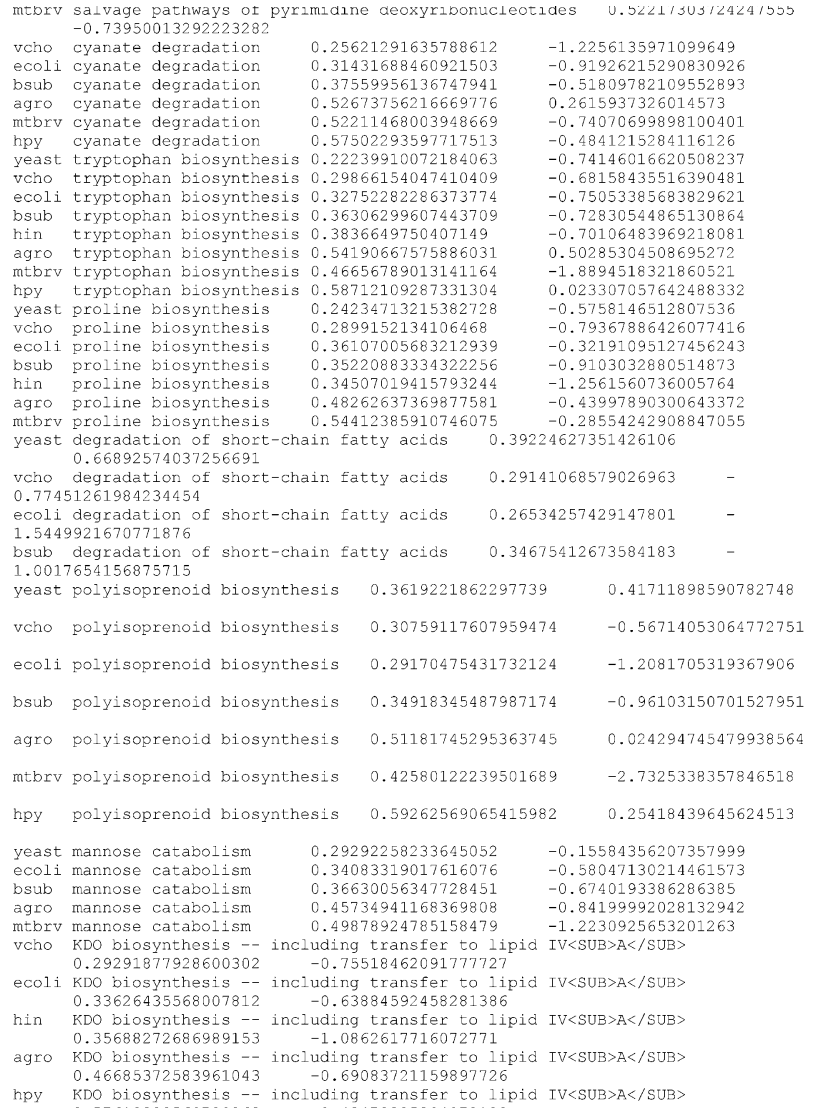

ecoli menaquinone biosynthesis

bsub menaquinone biosynthesis

hin menaquinone biosynthesis

mtbrv menaquinone biosynthesis

yeast phenylälanine biosynthesis

ecoli phenylalanine biosynthesis

bsub phenylalanine biosynthesis

hin phenylalanine biosynthesis

$0.263565 / 5298849 \$ 16$

0.3281952717278982

0.37694240641858306

0.38373144141672072

0.53119052434350755

0.20475614749307358

0.29226960983946604

0.40095690707369258

0.37966928792178506

vcho L-alanine degradation $\quad 0.2659337031260855$

ecoli L-alanine degradation 0.3082499050407122

$\begin{array}{lll}\text { bsub } & \text { L-alanine degradation } & 0.34048477586325421 \\ \text { hin } & \text { L-alanine degradation } & 0.39246919493528093\end{array}$

$\begin{array}{lll}\text { hin } & \text { L-alanine degradation } & 0.39246919493528093 \\ \text { agro I-alanine degradation } & 0.45531287835247203\end{array}$

mtbrv L-alanine degradation 0.49544644381915848

hpy L-alanine degradation 0.60422243819959975

0.9759390641156612

ecoli threonine biosynthesis from homoserine

0.81422539266305494

bsub threonine biosynthesis from homoserine
0.025487727536118589

hin threonine biosynthesis from homoserine

agro threonine biosynthesis from homoserine
0.29103327440030957 mtbrv threonine biosynthesis from homoserine
2.0644985601050592

hpy threonine biosynthesis from homoserine
0.33305984649658066

0.330598 trehalose degradation, low osmolarity
0.87518728547078395

vcho trehalose degradation, low osmolarity

0.6713232909810271

ecoli trehalose degradation, low osmolarity
1.005202705458355

1.0052027054458359

bsub trehalose degr
0.46674827202536839

$\begin{array}{ll}\text { yeast thiamin biosynthesis } & 0.19976889877851101 \\ \text { vcho thiamin biosynthesis } & 0.25495493131881619\end{array}$

ecoli thiamin biosynthesis

bsub thiamin biosynthesis

hin thiamin biosynthesis

agro thiamin biosynthesia

hpy thiamin biosynthesis

0.3023957253602698

0.38329779971414774
0.31068576927248609

0.31068576927248609

0.45791849665959566

ecoli pyridine nucleotide cycling 0.31528642008361674
$-1.1313183133620003$

$-0.74194218021091585$

$-0.49558158432977134$

$-0.70010888394307191$

$-0.55301245770379892$

$-0.88796465123806567$

$-1.2009535430382856$

$-0.75853299581139599$
$-1.1010302362419195$

$-0.99677812376592567$

-1.1068871410115568
-0.57443773693135869

-0.57443773693135869
-0.87439025267354331

$-1.2922239924830934$ 0.74058255283058205
.275694125607852

0.32253784696444654

0.404978317379939

0.3805626636414578

0.49199128954847254 0.45810362240305574

0.57862456185918187

0.20629487490178225

0.2994621740116935

0.30759053427673017

0.3786619953151511

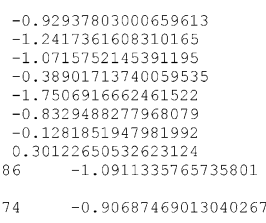

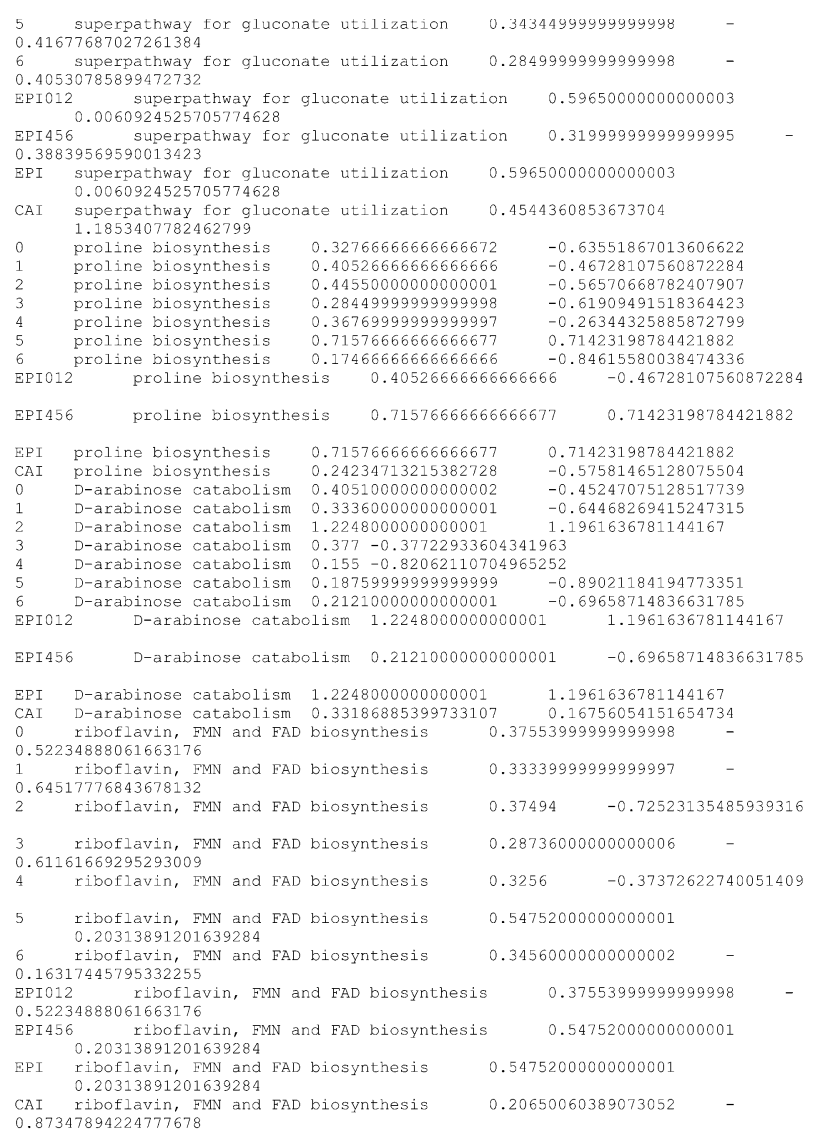

0.28876775885611078 andinopimelate biosynthesis 0.47435000000000005

0.28876775885611078
1 Yysine and diaminopimelate biosynthesis 0.45974999999999999 2.33241458932511359
lysine and diaminopimelate biosynthesis 0.90470000000000006
0.47246971669145699

lysine and diaminopimelate biosynthesis 0.39019999999999999

1ysine and diaminopimelate biosynthesis 0.19849999999999998
.7066707713829613

$\begin{aligned} & .7066707713829613 \\ & \text { lysine and diaminopimelate biosynthesis }\end{aligned} \quad 0.21245 \quad-0.81472349388147269$

$6 \quad$ Iysine and diaminopimelate biosynthesis $\quad 0.17845 \quad-0.83103911115581075$

EPI012 Iysine and diaminopimelate biosynthesis 0.90470000000000006

0.47246971669145699
EPI 566 lysine and diaminopimelate biosynthesis 0.19849999999999998

0.7066707713829613
EPI Iysine and diaminopinelate biosynthesis 0.90470000000000006

EPI lysine and diaminopimelate bis 0.47246971669145699
CAI

CAI lysine and diaminopimelate biosynthesis 0.36502964586112618
0.44292287299798455

oxidative branch of the pentose phosphate pathway 0.35294999999999999

-0.57575033330937708
oxidative branch of the pentose phosphate pathway

9032826972867731 of the pentose phosphate pathway

oxidative branch of the
-0.90426704622173038

-0.64668066502071853 pentose phosphate pathway

oxidative branch of the pentose phosphate pathway

-0.2955327212016467
oxidative branch of the pentose phosphate pathway

0.51715

0.29575000000000001

0.27394999999999997

0.35544999999999999

0.39400000000000002

-0.26321807772736311 pentose phosphate pathway 0.36465000000000003

oxidative branch of the
-0.087058265051692854

EPI012 oxidative branch of the pentose phosphate pathway 0.51715

0.19032826972867731 oxidative branch of the pentose phosphate pathway

EPI $\begin{aligned} & 0.36465000000000003 \\ & \text { oxidative branch of the pentose phosphate pathway }\end{aligned}$

CAI oxidative branch of the pentose phosphate pathway 0.42205103830715995

chorismate biosynthesis $0.52242500000000003-0.17512124100734008$ $\begin{array}{lll}\text { chorismate biosynthesis } & 0.52242500000000003 & -0.17512124100734008 \\ \text { chorismate biosynthesis } 0.4092875000000008 & -0.45732801968461106\end{array}$ chorismate biosynthesis $0.42275000000000007 \quad-0.61714073225510768$ $\begin{array}{lll}\text { chorismate biosynthesis } & 0.31353750000000002 & -0.54316873405624666 \\ \text { chorismate biosynthesis } & 0.33332500000000004 & -0.35349021951487747\end{array}$ $\begin{array}{lll}5 & \text { chorismate biosynthesis } 0.26648749999999999 & -0.65057051164281843 \\ 6 & \text { chorismate biosynthesis } 0.1745875000000001 & -0.84647211877169237 \\ \text { EPT012 chorismate biosynthesis } 0.52242500000000003 & -0.17512124100734008\end{array}$ EPI456 chorismate biosynthesis $0.33332500000000004 \quad-0.35349021951487747$

EPI chorismate biosynthesis 0.52242500000000003

CAI chorismate biosynthesis 0.30389965716727813 tryptophan biosynthesis 0.40523333333333328
tryptophan biosynthesis 0.35241666666666677
tryptophan biosynthesis 0.58086666666666664
$-0.17512124100734008$

$-0.064691549459460443$

-0.45215555900131899
-0.59810445523714872 


\begin{tabular}{|c|c|c|c|}
\hline & polyisoprenoid biosynthesis & 0.15440000000000001 & -0.99106549006845202 \\
\hline & polyisoprenoid biosynthesis & 0.21376666666666666 & -0.68992781390423308 \\
\hline & polyisoprenoid biosynthes & 0.63293333333333335 & - \\
\hline & $\begin{array}{l}\text { polyisoprenoid biosynth } \\
81390423308\end{array}$ & 0.21376666666666666 & 66 \\
\hline & polyisoprenoid biosynthesis & 0.63293333333333335 & -0.14195045070221066 \\
\hline & polyisoprenoid biosynthesis & 0.3619221862297739 & 0.41711898590782615 \\
\hline & phospholipid biosynthesis & 0.25961666666666666 & -0.79638493201040539 \\
\hline & phospholipid biosynthesis & 0.33353333333333329 & -0.64484771891390924 \\
\hline & phospholipid biosynthesis & 0.46983333333333333 & -0.51069298462312518 \\
\hline & phospholipid biosynthesis & 0.24809999999999999 & -0.71427228902909479 \\
\hline & phospholipid biosynthesis & 0.17759999999999998 & -0.76141932346190022 \\
\hline & phospholipid biosynthesis & 0.27663333333333334 & -0.61974990004970221 \\
\hline & phospholipid biosynthesis & 0.22776666666666665 & -0.63398940442272056 \\
\hline & phospholipid biosynthe & 0.46983333333333333 & 33 \\
\hline & phospholipid biosynt & 0.2766333333333 & 34 \\
\hline & $\begin{array}{l}\text { phospholipid biosynthesis } \\
\text { phos }\end{array}$ & 0.46983333333333333 & -0.51069298462312518 \\
\hline & phospholipid biosynthesis & 0.16756589234142572 & -1.1967870523740354 \\
\hline & 4-aminobutyrate degradation & 0.25840000000000002 & -0.79926106160061516 \\
\hline & $\begin{array}{l}\text { 4-aminobutyrate degradation } \\
\text { 4-aminobutyrate degradation }\end{array}$ & $\begin{array}{ll}0.15625 & -1.0836898157 \\
0.36780000000000002\end{array}$ & $\begin{array}{l}\frac{762766}{-0.74137373188082367} \\
-\end{array}$ \\
\hline & $\begin{array}{l}\text { 4-aminobutyrate degradation } \\
4 \text {-aminobutyrate degradation }\end{array}$ & $\begin{array}{l}0.2792 \quad-0.6329531591 \\
0.13275000000000001\end{array}$ & \\
\hline & 4-aminobutyrate degradation & 0.26584999999999998 & -0.65250708395236545 \\
\hline & $\begin{array}{l}\text { 4-aminobutyrate degradation } \\
\text { 4-aminobutyrate degradatio }\end{array}$ & $\begin{aligned}-0.247082 \\
800000000\end{aligned}$ & \\
\hline 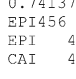 & $\begin{array}{l}8,73180826367 \\
4 \text {-aminobutyrate degradatio } \\
4 \text {-aminobutyrate degradation } \\
4 \text {-amininobutyrate degradation }\end{array}$ & $\begin{array}{l}\text { ion } 0.3246-0-0.2 \\
0.3246^{0.324}-0.2470820 \\
0.25791611381479673\end{array}$ & $\begin{array}{l}08207217559155 \\
17559155 \\
-0.44653212067758968\end{array}$ \\
\hline & 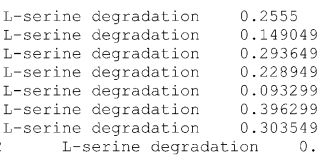 & $\begin{array}{ll}-0.8061164937745399 \\
499999999999 & -1.1015 \\
499999999997 & -0.0090 \\
499999999997 & -0.7643 \\
99999999999999994 & -0.8822 \\
99999999999999 & -0.2562 \\
4999999999999 & -0.311 \\
0.25559 & -0.8061164937\end{array}$ & 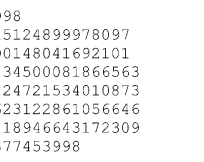 \\
\hline
\end{tabular}

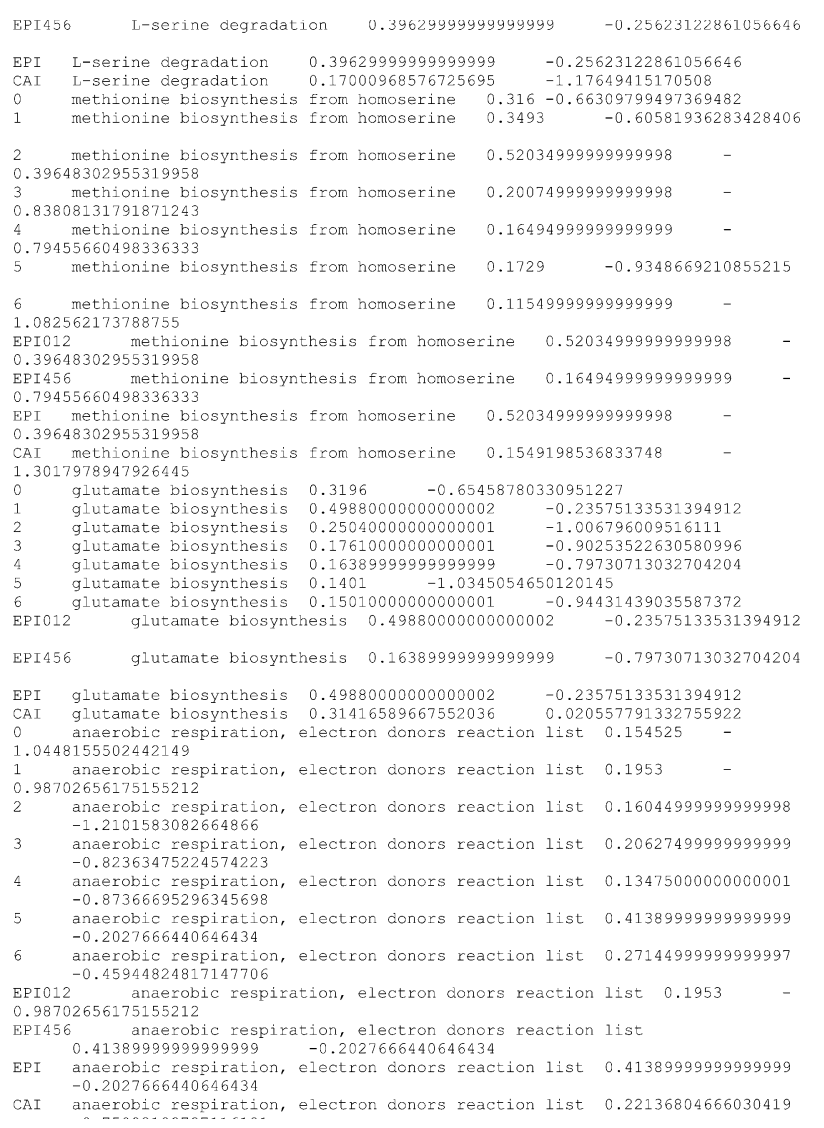

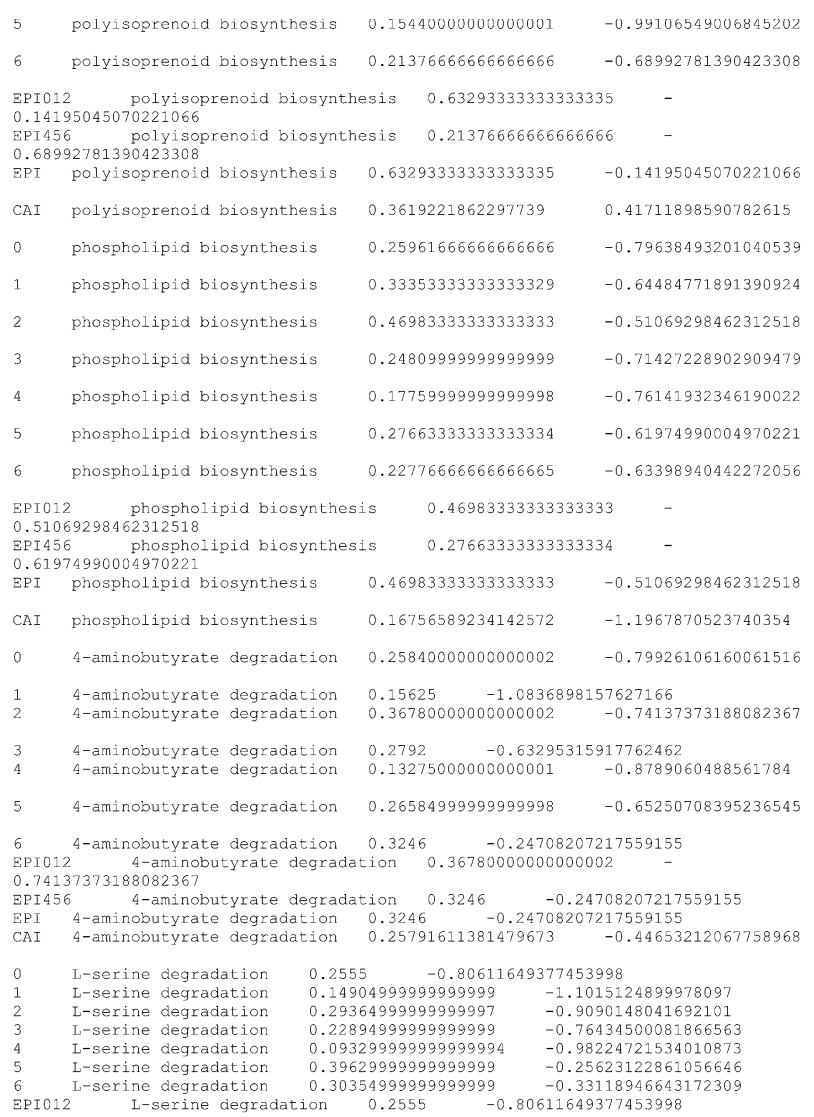

EPI456 L-serine degradation $0.39629999999999999 \quad-0.25623122861056646$ $\begin{array}{llll}\text { EPI } & \text { I-serine degradation } & 0.39629999999999999 & -0.25623122861056646 \\ \text { CAI } & \text { L-serine degradation } & 0.17000968576725695 & -1.17649415170508\end{array}$ $\begin{array}{llll}\text { CAI } & \text { L-serine degradation } & 0.17000968576725695 & -1.17649415170508 \\ 0 & \text { methionine biosynthesis from homoserine } & 0.316 & -0.66309799497369482\end{array}$ $\begin{array}{lll}\text { methionine biosynthesis from homoserine } & 0.316 & -0.66309799497369482 \\ \text { methionine biosynthesis from homoserine } & 0.3493 & -0.60581936283428406\end{array}$

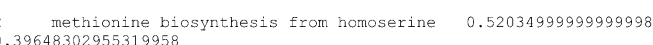
648302955319958
methionine biosynthesis from homoserine 0.20074999999999998 methionine biosynthesis from homoserine 0.16494999999999999
methionine biosynthesis from methionine biosynthos
methionine biosynthesis from homoserine $0.1729 \quad-0.9348669210855215$

6 methionine biosynthesis from homoserine 0.11549999999999999 1.082562173788755
EPI012 methionine biosynthesis from homoserine 0.52034999999999998

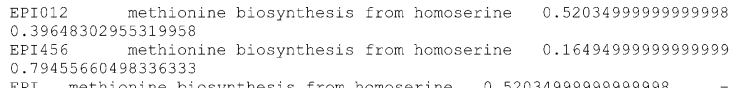
0.7945560498336333
EPI methionine biosynthesis from homoserine 0.52034999999999998
0.39648302955319958 0.39648302955319958
CAI methionine biosynthesis from homoserine 0.1549198536833748

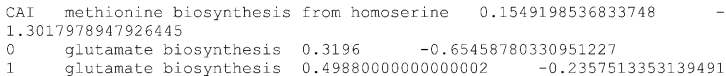
$\begin{array}{lll}\text { glutamate biosynthesis } & 0.49880000000000002 & -0.2357513353139491 \\ \text { glutamate biosynthesis } & 0.25040000000000001 & -1.006796009516111\end{array}$ $\begin{array}{lll}\text { glutamate biosynthesis } & 0.25040000000000001 & -1.006796009516111 \\ \text { glutamate biosynthesis } & 0.1761000000000001 & -0.902535263058096\end{array}$ $\begin{array}{llll}\text { glutamate biosynthesis } & 0.17618000000000001 & -0.90253522630580996 \\ \text { glutamate biosynthesis } & 0.1638999999999999 & -0.79730713032704204\end{array}$

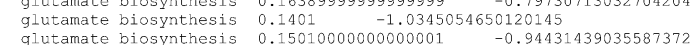
$\begin{array}{llll}6 & \text { glutamate biosynthesis } & 0.15010000000000001 & -0.94431439035587372 \\ \text { EPI012 } & \text { glutamate biosynthesis } & 0.4988000000000002 & -0.23575133531394912\end{array}$ EPI456 giutamate biosynthesis $\quad 0.16389999999999999 \quad-0.79730713032704204$

$\begin{array}{llll}\text { EPI } & \text { glutamate biosynthesis } & 0.49880000000000002 & -0.23575133531394912 \\ \text { CAI } & \text { glutamate biosynthesis } & 0.31416589667552036 & 0.020557791332755922\end{array}$ 0 anaerobic respiration, electron donors reaction list 0.154525 .0448155502442149

1.9 anaerobic respiration, electron donors reaction list 0.1953
0.98702656175155212 anaerobic respiration, electron donors reaction list 0.16044999999999998 $-1.2101583082664866$ $-1,2101583082664866$
anaerobic respiration, electron donors reaction list 0.20627499999999999
-0.82363475224574223

anaerobic respiration, electron donors reaction list 0.13475000000000001 -0.87366695296345698
anaerobic respiration, electron donors reaction list 0.41389999999999999 aneerobic respiration, electron donors reaction list 0.27144999999999997 and 0.45944824817147706

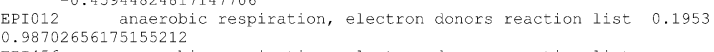

EPI 456 a
anaerobic respiration, electron donors reaction list

EPI anaerobic respiration, electron donors reaction list 0.41389999999999999

CAI anaerobic respiration, electron donors reaction list 0.22136804666030419
-0.75002188727116181 


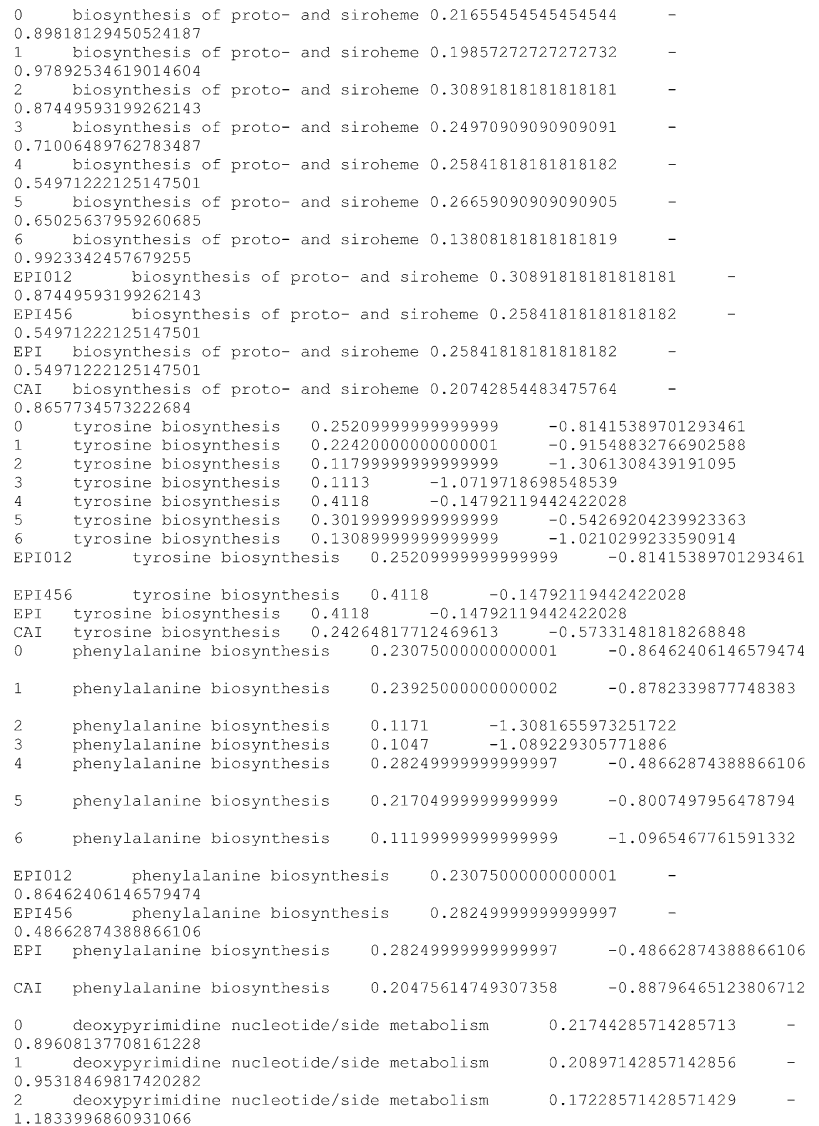

3 deoxypyrimidine nucleotide/side metabolism 0.92894433278490496 nucleotide/side metabolism 4.600099072280406607
0.6009

5 deoxypyrimidine nucleotide/side metabolism 0.91989510154952836

deoxypyrimidine nucleotide/side metabolism

EPI012 deoxypyrimidine nucleotide/side metabolism

EPI456 deoxypyrimidine nucleotide/side metabolism
-0.60099072280406607

EPI deoxypyrimidine nucleotide/side metabolism

EPI deoxypyrimidine nucleotide/side metabolism 0.23884285714285713

0 pantothenate and coenzyme A biosynthesis 0.22170000000000001

1) pantothenate and coenzyme A biosynthesis 0.20046666666666665

.9742371427402585

pantothenate and coenzyme A biosynthesis 0.264333333333333336
97529501234077021

pantothenate and coenzyme A biosynthesis 0.13066666666666668
0213326260781077

pantothenate and coenzyme A biosynthesis 0.20820000000000002

.68126115630326223

pantothenate and coenzyme A biosynthesis 0.16883333333333336
.94722048039348072

6 pantothenate and coenzyme A biosynthesis 0.109633333333333332

1.1060030310952937
EPI012 pantothenate and coenzyme A biosynthesis 0.22170000000000001

0.8860177377326981 panto and coenzyme A biosynthesis 0.20820000000000002

EPI456 pantothenate and coenzyme A biosynthesis 0.20820000000000002

EPI pantothenate and coenzyme A biosynthesis 0.20820000000000002

0.68126115630326223

CAI pantothenate and coenzyme A biosynthesis 0.1692359165118541

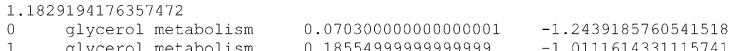

glycerol metabolism $0.18554999999999999 \quad-1.0111614331115741$

$\begin{array}{lll}\text { Glycerol metabolism } & 0.12740000000000001 & -1.2848789750113438 \\ \text { glycerol metabolism } & 0.1695500000000001 & -0.91966192407195579\end{array}$

glycerol metabolism $\quad 0.076049999999999993 \quad-1.0274344174148311$

glycerol metabolism $0.21995000000000001 \quad-0.79194029023974433$

$\begin{array}{llll}6 & \text { glycerol metabolism } & 0.1769999999999999 & -0.83683273213782461 \\ \text { EPI012 } & \text { glycerol metabolism } 0.1855499999999999 & -1.0111614331115741\end{array}$

EPI456 glycerol metabolism $0.21995000000000001 \quad-0.79194029023974433$

$\begin{array}{llll}\text { EPI } & \text { glycerol metabolism } & 0.21995000000000001 & -0.79194029023974433 \\ \text { CAI } & \text { glycerol metabolism } & 0.20549952823558804 & -0.88179172704259057\end{array}$

CAI Glycerol metabolism $\quad 0.20549952823558804 \quad-0.88179172704259057$

$\begin{array}{lll}\text { lactose degradation } & 0.06850000000000005 & -1.2481736718862431 \\ \text { lactose degradation } & 0.085300000000000001 & -1.2593174181210294\end{array}$

$\begin{array}{llll}\text { lactose degradation } & 0.085300000000000001 & -1.2593174181210294 \\ \text { lactose degradation } & 0.1709 & -1.1865325603849808\end{array}$

lactose degradation $\quad 0.051700000000000003 \quad-1.2278117457116904$

lactose degradation $\quad 0.1128 \quad-1.1174363262679063$

$\begin{array}{llll}6 & \text { lactose degradation } & 0.16109999999999999 & -0.900362782906 \\ \text { EPI012 } & \text { lactose degradation } & 0.1709 & -1.1865325603849808\end{array}$

EPI456 lactose degradation $0.16109999999999999-0.90036278290611393$

EPI lactose degradation

EPI lactose degradation

biotin biosynthesis

biotin biosynthesis

biotin biosynthesis

biotin biosynthesi

biotin biosynthesis

$\begin{array}{lllll}6 & \text { biotin biosynthesis } & 0.059249999999999997 & -1.3073147118841182 \\ \text { EPI012 biotin biosynthesis } 0.098275000000000001 & -1.1777872949970669\end{array}$

0.16109999999999999

. $0.098275000000000001--1.8777872949970669$

$\begin{array}{ll}0.098275000000000001 & -1.1777872949970669 \\ 0.0851499999999999 & -1.2596887238342607\end{array}$

$0.071124999999999994 \quad-1.412107583818207$

$0.06825-1.182772452731254$

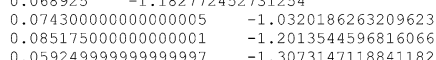

EPI456

biotin biosynthesis

0.0743000000000000005

$-1.0320186263209623$

$\begin{array}{llll}\text { EPI biotin biosynthesis } & 0.074300000000000005 & -1.0320186263209623 \\ \text { CAI biotin biosvnthesis } & 0.18262596579645649 & -1.0717304205573919\end{array}$ 